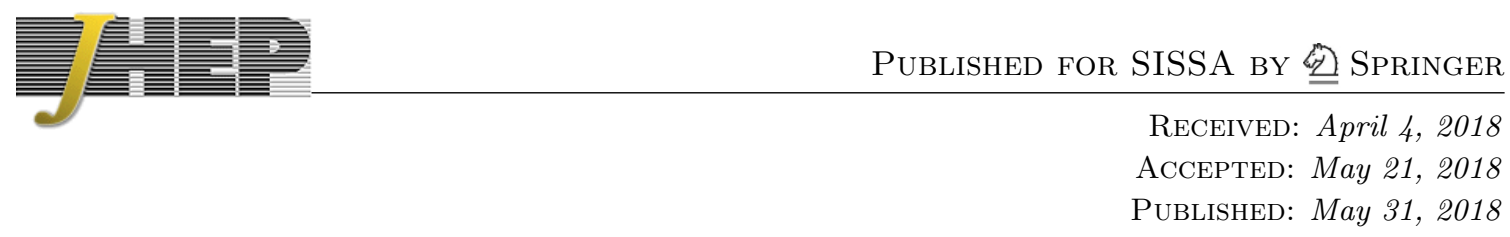

\title{
Holographic anisotropic background with confinement-deconfinement phase transition
}

\author{
Irina Aref'eva ${ }^{a}$ and Kristina Rannu ${ }^{a, b}$ \\ ${ }^{a}$ Steklov Mathematical Institute, Russian Academy of Sciences, \\ Gubkina str. 8, 119991, Moscow, Russia \\ ' Peoples' Friendship University of Russia, \\ Miklukho-Maklaya str. 6, 117198, Moscow, Russia \\ E-mail: arefeva@mi.ras.ru, rannukristina@gmail.com
}

ABSTRACT: We present new anisotropic black brane solutions in 5D Einstein-dilaton-twoMaxwell system. The anisotropic background is specified by an arbitrary dynamical exponent $\nu$, a nontrivial warp factor, a non-zero dilaton field, a non-zero time component of the first Maxwell field and a non-zero longitudinal magnetic component of the second Maxwell field. The blackening function supports the Van der Waals-like phase transition between small and large black holes for a suitable first Maxwell field charge. The isotropic case corresponding to $\nu=1$ and zero magnetic field reproduces previously known solutions. We investigate the anisotropy influence on the thermodynamic properties of our background, in particular, on the small/large black holes phase transition diagram.

We discuss applications of the model to the bottom-up holographic QCD. The RG flow interpolates between the UV section with two suppressed transversal coordinates and the IR section with the suppressed time and longitudinal coordinates due to anisotropic character of our solution. We study the temporal Wilson loops, extended in longitudinal and transversal directions, by calculating the minimal surfaces of the corresponding probing open string world-sheet in anisotropic backgrounds with various temperatures and chemical potentials. We find that dynamical wall locations depend on the orientation of the quark pairs, that gives a crossover transition line between confinement/deconfinement phases in the dual gauge theory. Instability of the background leads to the appearance of the critical points $\left(\mu_{\vartheta, b}, T_{\vartheta, b}\right)$ depending on the orientation $\vartheta$ of quark-antiquark pairs in respect to the heavy ions collision line.

KEYWORDS: Holography and quark-gluon plasmas, AdS-CFT Correspondence, Gaugegravity correspondence

ArXiv ePrint: 1802.05652 


\section{Contents}

1 Introduction 1

2 Black brane anisotropic solutions 3

2.1 The equations of motion and boundary conditions 3

2.2 Solutions with factor $b(z)=\exp P(z)$ and spatial anisotropy 4

2.3 Solutions with factor $b(z)=\exp \left(c z^{2} / 2\right)$ and spatial anisotropy $\quad 7$

$\begin{array}{lll}2.3 .1 & \text { Blackening function } & 7\end{array}$

$\begin{array}{ll}\text { 2.3.2 Coupling function } f_{2} & 10\end{array}$

$\begin{array}{lll}2.3 .3 & \text { Scalar field } & 12\end{array}$

$\begin{array}{lll}2.3 .4 & \text { Scalar potential } & 15\end{array}$

$\begin{array}{ll}2.4 \text { Scalar invariants } & 16\end{array}$

$\begin{array}{lll}2.5 & \text { RG flow } & 17\end{array}$

3 Thermodynamics of the background 22

3.1 Temperature 22

$\begin{array}{lll}3.2 & \text { Entropy } & 26\end{array}$

$\begin{array}{lll}3.3 & \text { Free energy } & 30\end{array}$

4 Confinement-deconfinement phase transition $\quad 33$

4.1 Equation for the dynamical wall 33

$4.2 \quad P(z)=c z^{2} / 2 \quad 36$

4.2.1 Zero temperature $\quad 36$

4.2.2 Non-zero temperature, zero chemical potential $\quad 37$

$\begin{array}{lll}\text { 4.2.3 Non-zero chemical potential } & 39\end{array}$

4.2.4 The dynamical wall position $\quad 40$

4.2.5 Phase transition lines and critical points 41

5 Conclusion and discussion $\quad 43$

$\begin{array}{ll}\text { A Equations of motion } & 48\end{array}$

B Simplest solutions to system (2.5)-(2.10) $\mathbf{5 0}$

B.1 Solutions for anisotropic metric $\nu \neq 1$ and $c=0, A_{t} \neq 0 \quad 50$

B.2 Solutions for anisotropic metric $\nu \neq 1$ and $c=0, A_{t}=0 \quad 51$

B.3 Vacuum solutions $\quad 52$ 


\section{Introduction}

Study of the phase diagram of QCD, as a function of temperature $\mathrm{T}$ and chemical potential $\mu$, corresponding to baryon density or some other conserved charge, is one of the great modern challenge. The diagram of QCD displays a rich structure $[1,2]$. Particularly interesting and important features of the phase diagram are the nature of the chiral phase transition, the location of the chiral critical point and its properties. There are well known obstacles to study this problem by usual tools, such as perturbative theory or lattice calculations.

The gravity/gauge duality provides an alternative tool for understanding dynamics of the strong coupling system, where standard methods are not enough. One such system is the quark-gluon plasma (QGP) produced in the heavy-ions-collisions (HIC) [3-5]. It is believed that the QGP is created in a very short time after the collision $\left(\tau_{\text {therm }} \approx\right.$ few $0.1 \mathrm{fm} / c$ ) and there are indications that during this time the QGP is anisotropic [6]. In [7] we have considered a special anisotropic backgrounds, parametrized by the dynamical exponent $\nu$, and found out that the shock wave model of HIC for $\nu=4.45$ reproduces the experimental energy dependence of the total multiplicity, $\mathcal{M} \sim s^{0.155}$ [8]. Note that all attempts to reproduce this dependence in isotropic models failed [9-21].

On the other hand, static holographic models perfectly reproduce main properties of QCD [3-5, 22]. Therefore, it would be interesting to know how these properties are changed in anisotropic backgrounds. Some of these questions have been already addressed in [23-36] and refs therein. In particular, in [35] the confinement-deconfinement crossover transition in the temperature-chemical potential plane has been observed. However, in this paper, analogous to [37] and many others [38-43], it was not assumed that the metric is supported by some Lagrangian. Let us remind, that for the isotropic case, $\nu=1$, the background used in [37] was constructed in [44] as a solution of equations of motion in the Einstein-dilaton theory. Later, this background has been generalized for non-zero electro-field $[45,46]$ in the Einstein-dilaton-Maxwell theory in order to describe the confinement-deconfinement phase transition holographically, (see also [42, 43, 47-49] and early papers [50-52]). The anisotropic $\nu \neq 1$ black brane background with a trivial warp factor and a non-zero electromagnetic field has been constructed in [33]. As it already has been mentioned, the collision of shock waves in this 5-dimensional dual vacuum background gives the total multiplicity dependence on energy in the form $\mathcal{M} \sim s^{0.155}$, which reproduces the experimental dependence [8].

This paper is devoted to the construction of the anisotropic 5-dimensional background specified by an arbitrary dynamical exponent $\nu$, a nontrivial warp factor, a non-zero time component of the first Maxwell field and a non-zero longitudinal magnetic component of the second Maxwell field. To see clearly possible new effects caused by anisotropy, it is useful to deal with an explicit analytical solution. For this purpose we take the particular case of the simplest warp factor $b(z)=e^{\frac{c z^{2}}{2}}$. We find the dilaton potential by the potential reconstruction method similar to the isotropic case [46] (and refs therein). We show that only $c \leq 0$ guarantees real solutions for the dilaton (compare with [19] and refs therein). We construct the blackening function that supports the Van der Waals-like phase 
transition between small and large black holes for a suitable first Maxwell field charge. The isotropic case corresponding to $\nu=1$ and zero magnetic field reproduces previously known solutions [45, 46]. We investigate the anisotropy influence on the thermodynamic properties of our background, in particular, on the small/large black holes phase transition diagram. We find that the anisotropy changes the location of the domain of instability.

We also discuss applications of the model to the bottom-up holographic QCD. We note that the RG flow interpolates between the UV section with two transversal suppressed coordinates and the IR section with the suppressed time and longitudinal coordinates due to anisotropic character of our solution. We study the temporal Wilson loops extended in longitudinal and transversal directions by calculating the minimal surfaces of the corresponding probing open string world-sheet for various values of temperature and chemical potential. We find that for particular sets of the model parameters the dynamical wall appears. The appearance of dynamical walls also depends on the orientation of the temporal Wilson loop, that gives a crossover transition line between confinement-deconfinement phases in the dual gauge theory. This effect has been also observed in the anisotropic model considered in [35]. In the background, investigated in the present paper, there are two more anisotropic effects. Namely, the instability of the background restricts leads to the appearance of critical points $\left(\mu_{\vartheta, b}, T_{\vartheta, b}\right)$. Each critical point is located at intersection of the confinement/deconfinement open string phase transition line and the small/large black holes phase transition line of our background. The lines of the first type depend on orientation of the quark-antiquark pairs and the lines of the second type are fixed for the given anisotropy parameter $\nu$. In other words, positions of the critical points $\left(\mu_{\vartheta, b}, T_{\vartheta, b}\right)$ depend on the orientation of quark-antiquark pair in respect to the heavy ions collision line. Averaging on all possible orientations on the quark-antiquark pairs, one gets a family of the critical points. In our model it also happens, that the confinement/deconfinement transition line, oriented along the transversal direction, is below the small/large black holes phase transition line. This means that near the small chemical potential the small/large black hole transition line is hidden by the confinement/deconfinement transition line for the pair of quarks oriented in the transverse direction. Recall that a small/large black holes transition line near the top of the holographic phase diagram is treated as a problem, since this behavior is not supported neither by experimental data nor by calculations performed in the framework of effective theories. Let us also remind, that most of the effective models suggest the existence of a QCD critical point $\left(\mu_{C E P}, T_{C E P}\right)$ somewhere in the middle of the phase diagram, where the crossover line becomes a first order transition line. There were attempts to relate $\left(\mu_{C E P}, T_{C E P}\right)$ with the small/large black holes background transition [51,52], but here there is a problem with the first order phase transition in the top of the QCD phase diagram, that is not present at the QCD phase diagram $[1,2]$. The isotropic holographic model improving this has been proposed in [49] just by removing the small/large black holes background transition, see also [53, 54]. We note, that the presence of the small/large black holes background transition endows our anisotropic model by a rich phase structure.

The paper is organized as follows. In section 2 we construct the anisotropic 5-dimensional solution with an arbitrary dynamical exponent $\nu$, a nontrivial warp factor, a non-zero 
time component of the first Maxwell field and a non-zero longitudinal magnetic component of the second Maxwell field. In section 2.3 we consider exponential warp factors with quadratic exponent and show that only negative definite quadratic form guarantees the real solutions for the dilaton. In section 3 we discuss the thermodynamics of the constructed background and find out the small/large black holes transition line in the $(\mu, T)$-plane. Sections 2.5 and 4 are devoted to applications to QCD. In section 2.5 we shortly discuss the RG flows corresponding to constructed solutions. In section 4.1 we find dynamical walls corresponding to the temporal Wilson loops extended in the longitudinal and transversal directions. In section 4.2.5 we determine the relative position of the background and the confinement-deconfinement phase transition lines and discuss the corresponding critical points. In appendix A we derive E.O.M. and in appendix B we present simplest solutions, the black hole solutions for $c=0$, with zero and non-zero chemical potential, and the vacuum solution for $c<0$ for completeness.

\section{Black brane anisotropic solutions}

\subsection{The equations of motion and boundary conditions}

We consider a 5-dimensional Einstein-dilaton-two-Maxwell system. In the Einstein frame the action of the system is specified as

$$
S=\int \frac{d^{5} x}{16 \pi G_{5}} \sqrt{-\operatorname{det}\left(g_{\mu \nu}\right)}\left[R-\frac{f_{1}(\phi)}{4} F_{(1)}^{2}-\frac{f_{2}(\phi)}{4} F_{(2)}^{2}-\frac{1}{2} \partial_{\mu} \phi \partial^{\mu} \phi-V(\phi)\right]
$$

where $F_{(1)}^{2}$ and $F_{(2)}^{2}$ are the squares of the Maxwell fields $F_{\mu \nu}^{(1)}=\partial_{\mu} A_{\nu}-\partial_{\nu} A_{\mu}$ and $F_{\mu \nu}^{(2)}=q d y^{1} \wedge d y^{2}, f_{1}(\phi)$ and $f_{2}(\phi)$ are the gauge kinetic functions associated with the corresponding Maxwell fields, $V(\phi)$ is the potential of the scalar field $\phi$.

We search the black brane solution in the anisotropic background. For this purpose we use the metric ansatz in the following form:

$$
\begin{aligned}
d s^{2} & =\frac{L^{2} b(z)}{z^{2}}\left[-g(z) d t^{2}+d x^{2}+z^{2-\frac{2}{\nu}}\left(d y_{1}^{2}+d y_{2}^{2}\right)+\frac{d z^{2}}{g(z)}\right] \\
\phi & =\phi(z), \quad A_{\mu}^{(1)}=A_{t}(z) \delta_{\mu}^{0}, \\
F_{\mu \nu}^{(2)} & =q d y^{1} \wedge d y^{2}
\end{aligned}
$$

where $b(z)$ is the warp factor and $g(z)$ is the blackening function; we set the AdS radius $L=1$ and all the quantities in formulas and figures are presented in dimensionless units.

The variation of the action (2.1) over metric components $g_{\mu \nu}$ gives 4 independent equations, corresponding to 00-, 11-, 22- and 44-components of the Einstein tensor, that 
are presented in the appendix A. These equations can be transformed to the following ones:

$$
\begin{array}{r}
g^{\prime \prime}+g^{\prime}\left(\frac{3 b^{\prime}}{2 b}-\frac{1}{z}-\frac{2}{\nu z}\right)-\frac{z^{2}}{b} f_{1} A_{t}^{\prime 2}=0, \\
b^{\prime \prime}-\frac{3\left(b^{\prime}\right)^{2}}{2 b}+\frac{2 b^{\prime}}{z}-\frac{4 b}{3 \nu z^{2}}\left(1-\frac{1}{\nu}\right)+\frac{b}{3}\left(\phi^{\prime}\right)^{2}=0 \\
2 g^{\prime}\left(1-\frac{1}{\nu}\right)+g\left(1-\frac{1}{\nu}\right)\left(\frac{3 b^{\prime}}{b}-\frac{4}{z}-\frac{4}{\nu z}\right)+\frac{q^{2} z^{-1+\frac{4}{\nu}}}{b} f_{2}=0 \\
-V-\frac{z^{4}}{2 b^{2}} A_{t}^{\prime 2} f_{1}-\frac{3 z^{2} b^{\prime} g^{\prime}}{2 b^{2}}-\frac{3 z^{2} g b^{\prime 2}}{b^{3}}+\frac{9 z g b^{\prime}}{2 \nu b^{2}}+\frac{15 z g b^{\prime}}{2 b^{2}}+\frac{z g^{\prime}}{\nu b}+\frac{2 z g^{\prime}}{b}+ \\
+\frac{z^{2} g \phi^{\prime 2}}{2 b}-\frac{8 g}{\nu b}-\frac{4 g}{b}=0
\end{array}
$$

Here and below ${ }^{\prime}=d / d z$. The variation of the action (2.1) over the scalar field $\phi$ and the components $A_{\mu}^{(1)}$ of the first Maxwell field leads to the following EOM:

$$
\begin{aligned}
\phi^{\prime \prime}+\phi^{\prime}\left(\frac{g^{\prime}}{g}+\frac{3 b^{\prime}}{2 b}-\frac{\nu+2}{\nu z}\right)+\frac{z^{2} A_{t}^{\prime 2}}{2 b g} \frac{\partial f_{1}}{\partial \phi}-\frac{q^{2} z^{-2+\frac{4}{\nu}}}{2 b g} \frac{\partial f_{2}}{\partial \phi}-\frac{b}{z^{2} g} \frac{\partial V}{\partial \phi} & =0 \\
A_{t}^{\prime \prime}+A_{t}^{\prime}\left(\frac{b^{\prime}}{2 b}+\frac{f_{1}^{\prime}}{f_{1}}-\frac{2-\nu}{\nu z}\right) & =0
\end{aligned}
$$

The EOM for the second Maxwell field doesn't give any contribution into system (2.5)(2.10) as its left-hand side is identically zero:

$$
\partial_{\mu}\left(\sqrt{-g} f_{2}(\phi) F_{(2)}^{\mu \nu}\right)=\partial_{4}\left(\sqrt{-g} f_{2}(\phi) F_{(2)}^{4 \nu}\right) \equiv 0 .
$$

We also impose the boundary conditions in the form:

$$
\begin{aligned}
b(0) & =1 \\
g(0) & =1 \quad \text { and } \quad g\left(z_{h}\right)=0 \\
A_{t}(0) & =\mu \quad \text { and } \quad A_{t}\left(z_{h}\right)=0
\end{aligned}
$$

where $z_{h}$ is the horizon. As to the scalar field, it is natural to require that $\phi(z)$ is real for $0<z \leq z_{h}$ and that

$$
\phi\left(z_{h}\right)=0
$$

\subsection{Solutions with factor $b(z)=\exp P(z)$ and spatial anisotropy}

One can use the following strategy to find particular solutions of the system of equations $(2.5)-(2.10)$.

- Choose the form of functions $b(z)$ and $f_{1}(z)$.

- Using these $b(z)$ and $f_{1}(z)$, find the time component of the electric field $A_{t}(z)$ from (2.10).

- Using $f_{1}(z), b(z)$ and $A_{t}^{\prime}(z)$, get the blackening function $g(z)$ from (2.5). 
- Using $b(z)$, find the derivative of the scalar field $\phi^{\prime}(z)$ from (2.6). To have a solution one has to be sure that

$$
b^{\prime \prime}-\frac{3 b^{\prime 2}}{2 b}+\frac{2 b^{\prime}}{z}-\frac{4 b(\nu-1)}{3 \nu^{2} z^{2}} \leq 0 .
$$

- Using $g(z)$ and $b(z)$, get $f_{2}(z)$ from $(2.7)$.

- Finally, get $V=V(z)$ from (2.8).

Let us express the warp factor $b(z)$ via a polynomial $P(z):^{1}$

$$
b(z)=e^{P(z)},
$$

and take the coupling factor $f_{1}(z)$ :

$$
f_{1}(z)=z^{-2+\frac{2}{\nu}}
$$

In this case the equation (2.10) becomes

$$
A_{t}^{\prime \prime}+A_{t}^{\prime}\left(\frac{P^{\prime}(z)}{2}-\frac{1}{z}\right)=0
$$

and it's solution has the form

$$
A_{t}=C_{1} \int_{0}^{z} e^{-\frac{P(\xi)}{2}} \xi d \xi+C_{2} .
$$

If we take into account the boundary conditions (2.13), the integration constants equal to

$$
C_{1}=-\frac{\mu}{\int_{0}^{z_{H}} e^{-\frac{P(\xi)}{2}} \xi d \xi}=-\tilde{\mu}, \quad C_{2}=\mu
$$

and the solution (2.19) becomes

$$
A_{t}=\mu\left(1-\frac{\int_{0}^{z} e^{-\frac{P(\xi)}{2}} \xi d \xi}{\int_{0}^{z_{H}} e^{-\frac{P(\xi)}{2}} \xi d \xi}\right)=\tilde{\mu} \int_{z}^{z_{H}} e^{-\frac{P(\xi)}{2}} \xi d \xi .
$$

In a similar way equation (2.5) takes the form

$$
g^{\prime \prime}+g^{\prime}\left(\frac{3}{2} P^{\prime}(z)-\frac{1}{z}-\frac{2}{\nu z}\right)-\tilde{\mu} e^{-2 P(z)} z^{2+\frac{2}{\nu}}=0,
$$

and its solution is:

$$
g=C_{4}+\int_{0}^{z} e^{-\frac{3 P(\xi)}{2}}\left(C_{3}+\tilde{\mu}^{2} \int_{0}^{\xi} e^{-\frac{P(\chi)}{2}} \chi d \chi\right) \xi^{1+\frac{2}{\nu}} d \xi .
$$

\footnotetext{
${ }^{1}$ For the isotropic case this form of factor $b$ has been considered, in particular, in $[45,46]$, other form of this factor has been also considered in [55].
} 
Using the first boundary condition in (2.12) we can determine the integration constant $C_{4}=1$, and taking into account the boundary condition on the horizon we find $C_{3}$

$$
C_{3}=-\frac{1+\tilde{\mu}^{2} \int_{0}^{z_{H}} e^{-\frac{3 P(\xi)}{2}}\left(\int_{0}^{\xi} e^{-\frac{P(\chi)}{2}} \chi d \chi\right) \xi^{1+\frac{2}{\nu}} d \xi}{\int_{0}^{z_{H}} e^{-\frac{3 P(\xi)}{2}} \xi^{1+\frac{2}{\nu}} d \xi},
$$

therefore the solution (2.23) becomes:

$$
\begin{aligned}
g= & 1+\tilde{\mu}^{2} \int_{0}^{z} e^{-\frac{3 P(\xi)}{2}}\left(\int_{0}^{\xi} e^{-\frac{P(\chi)}{2}} \chi d \chi\right) \xi^{1+\frac{2}{\nu}} d \xi \\
& -\frac{1+\tilde{\mu}^{2} \int_{0}^{z_{H}} e^{-\frac{3 P(\xi)}{2}}\left(\int_{0}^{\xi} e^{-\frac{P(\chi)}{2}} \chi d \chi\right) \xi^{1+\frac{2}{\nu}} d \xi}{\int_{0}^{z_{H}} e^{-\frac{3 P(\xi)}{2}} \xi^{1+\frac{2}{\nu}} d \xi} \int_{0}^{z} e^{-\frac{3 P(\xi)}{2}} \xi^{1+\frac{2}{\nu}} d \xi
\end{aligned}
$$

Equation (2.7) gives the following expression for the coupling function $f_{2}(z)$ :

$$
\begin{aligned}
& f_{2}(z)=-\frac{\nu-1}{q^{2} \nu} e^{P(z)} z^{1-\frac{4}{\nu}}\left[2 e^{-\frac{3 P(z)}{2}} z^{1+\frac{2}{\nu}}\left(C_{3}+\tilde{\mu}^{2} \int_{0}^{z} e^{-\frac{P(\xi)}{2}} \xi d \xi\right)\right. \\
& \left.+\frac{3 z \nu P^{\prime}(z)-4(\nu+1)}{z \nu}\left(1+C_{3} \int_{0}^{z} e^{-\frac{3 P(\xi)}{2}} \xi^{1+\frac{2}{\nu}} d \xi+\tilde{\mu}^{2} \int_{0}^{z} e^{-\frac{3 P(\xi)}{2}}\left(\int_{0}^{\xi} e^{-\frac{P(\chi)}{2}} \chi d \chi\right) \xi^{1+\frac{2}{\nu}} d \xi\right)\right] .
\end{aligned}
$$

Equation (2.6) allows to find the scalar field $\phi(z)$ :

$$
\phi(z)=C_{5}+\int_{0}^{z} \sqrt{-3 P^{\prime \prime}(\xi)+\frac{3}{2} P^{\prime 2}(\xi)-\frac{6}{\xi} P^{\prime}(\xi)+4 \frac{\nu-1}{\xi^{2} \nu^{2}}} d \xi .
$$

Using the boundary condition (2.14), we get

$$
C_{5}=-\int_{0}^{z_{h}} \sqrt{-3 P^{\prime \prime}(\xi)+\frac{3}{2} P^{\prime 2}(\xi)-\frac{6}{\xi} P^{\prime}(\xi)+4 \frac{\nu-1}{\xi^{2} \nu^{2}}} d \xi
$$

and therefore

$$
\phi(z)=\int_{z_{h}}^{z} \sqrt{-3 P^{\prime \prime}(\xi)+\frac{3}{2} P^{\prime 2}(\xi)-\frac{6}{\xi} P^{\prime}(\xi)+4 \frac{\nu-1}{\xi^{2} \nu^{2}}} d \xi .
$$

Finally, equation (2.8) gives the scalar potential $V(\phi(z))$ :

$$
\begin{aligned}
V(\phi(z)) & =\frac{9}{2} P^{\prime}(z) e^{-P(z)} z\left[1+\frac{1}{\nu}-C_{3}\left(\frac{e^{-\frac{3 P(z)}{2}}}{2} z^{2+\frac{2}{\nu}}-\left(1+\frac{1}{\nu}\right) \int_{0}^{z} e^{-\frac{3 P(\xi)}{2}} \xi^{1+\frac{2}{\nu}} d \xi\right)\right. \\
& \left.+\tilde{\mu}^{2}\left(\frac{e^{-\frac{3 P(z)}{2}}}{2} z^{2+\frac{2}{\nu}} \int_{0}^{z} e^{-\frac{P(\xi)}{2}} \xi d \xi-\left(1+\frac{1}{\nu}\right) \int_{0}^{z} e^{-\frac{3 P(\xi)}{2}}\left(\int_{0}^{\xi} e^{-\frac{P(\chi)}{2}} \chi d \chi\right) \xi^{1+\frac{2}{\nu}} d \xi\right)\right] \\
& +\frac{3}{2} e^{-P(z)} z^{2}\left(P^{\prime \prime}(z)+\frac{3}{2} P^{\prime 2}(z)+4 \frac{1+3 \nu+2 \nu^{2}}{2 z^{2} \nu^{2}}\right) \\
& \times\left[1+C_{3} \int_{0}^{z} e^{-\frac{3 P(\xi)}{2}} \xi^{1+\frac{2}{\nu}} d \xi+\tilde{\mu}^{2} \int_{0}^{z} e^{-\frac{3 P(\xi)}{2}}\left(\int_{0}^{\xi} e^{-\frac{P(\chi)}{2}} \chi d \chi\right) \xi^{1+\frac{2}{\nu}} d \xi\right] \\
& +e^{-\frac{5 P(z)}{2}} z^{2+\frac{2}{\nu}}\left[\left(\frac{3}{4} P^{\prime}(z) z+2+\frac{1}{\nu}\right)\left(C_{3}+\tilde{\mu}^{2} \int_{0}^{z} e^{-\frac{P(\xi)}{2}} \xi d \xi\right)-\tilde{\mu}^{2} e^{-\frac{P(z)}{2}} \frac{z^{2}}{2}\right] .
\end{aligned}
$$


If we substitute the expressions (2.16), (2.17), (2.21) and (2.25)-(2.30) into the constraint (2.9) and take into account that $\partial V / \partial \phi=V^{\prime} / \phi^{\prime}$, we can make certain that the left-hand side of (2.9) disappears. Therefore the system (2.5)-(2.10) is self-consistent and satisfied by the general solution (2.16), (2.17), (2.21) and (2.25)-(2.30).

\subsection{Solutions with factor $b(z)=\exp \left(c z^{2} / 2\right)$ and spatial anisotropy}

As we are interested in effects that can be caused by the anisotropy of the chosen metric ansatz, it is needed to find some particular solution of the system (2.5)-(2.10) and investigate it's properties explicitly. For this purpose we preferred to start from the simplest form of the warp-factor, the same as in [37]:

$$
b(z)=e^{\frac{c z^{2}}{2}}
$$

and take the factor $f_{1}(z)$ :

$$
f_{1}=z^{-2+\frac{2}{\nu}}
$$

In this case the equation (2.5) becomes

$$
A_{t}^{\prime \prime}+A_{t}^{\prime}\left(\frac{c z}{2}-\frac{1}{z}\right)=0
$$

and together with the boundary conditions

$$
A_{t}(0)=\mu, \quad A_{t}\left(z_{h}\right)=0
$$

this gives

$$
A_{t}(z)=\mu \frac{e^{-\frac{c z^{2}}{4}}-e^{-\frac{c z_{h}^{2}}{4}}}{1-e^{-\frac{c z_{h}^{2}}{4}}} .
$$

\subsubsection{Blackening function}

Using the solution (2.35) and $f_{1}$ given by (2.17), we rewrite equation (2.5) for the blackening function:

$$
g^{\prime \prime}+g^{\prime}\left(\frac{3 c z}{2}-\frac{1}{z}-\frac{2}{\nu z}\right)-\frac{\mu^{2} c^{2} z^{2+\frac{2}{\nu}} e^{-c z^{2}}}{4\left(1-e^{-\frac{c z_{H}^{2}}{4}}\right)^{2}}=0 .
$$

Taking into account the boundary conditions (2.12), we get

$$
\begin{aligned}
g(z)= & 1-\frac{\gamma\left(1+\frac{1}{\nu} ; \frac{3}{4} c z^{2}\right)}{\gamma\left(1+\frac{1}{\nu} ; \frac{3}{4} c z_{h}^{2}\right)}-\frac{\mu^{2} e^{\frac{1}{2} c z_{h}^{2}}}{4 c^{1 / \nu}} \frac{\gamma\left(1+\frac{1}{\nu} ; c z^{2}\right)}{\left(1-e^{\frac{1}{4} c z_{h}^{2}}\right)^{2}} \\
& +\frac{\mu^{2} e^{\frac{1}{2} c z_{h}^{2}}}{4 c^{1 / \nu}} \frac{\gamma\left(1+\frac{1}{\nu} ; c z_{h}^{2}\right)}{\left(1-e^{\frac{1}{4} c z_{h}^{2}}\right)^{2}} \frac{\gamma\left(1+\frac{1}{\nu} ; \frac{3}{4} c z^{2}\right)}{\gamma\left(1+\frac{1}{\nu} ; \frac{3}{4} c z_{h}^{2}\right)},
\end{aligned}
$$

where $\gamma\left(1+\frac{1}{\nu}, x\right)$ is the incomplete gamma function. 
There is no problem with solution in such a form for $c>0$, but for $c<0$ some ingredients of this presentation seem to fail. Indeed, $c^{1 / \nu}$ is ill-defined for $c<0$. By this reason we rewrite the above formula as

$$
g(z)=1-\frac{z^{2+\frac{2}{\nu}}}{z_{h}^{2+\frac{2}{\nu}}} \frac{\mathfrak{G}\left(\frac{3}{4} c z^{2}\right)}{\mathfrak{G}\left(\frac{3}{4} c z_{h}^{2}\right)}-\frac{\mu^{2} c z^{2+\frac{2}{\nu}} e^{\frac{c z_{h}^{2}}{2}}}{4\left(1-e^{\frac{c z_{h}^{2}}{4}}\right)^{2}} \mathfrak{G}\left(c z^{2}\right)+\frac{\mu^{2} c z^{2+\frac{2}{\nu}} e^{\frac{c z_{h}^{2}}{2}}}{4\left(1-e^{\frac{c z_{h}^{2}}{4}}\right)^{2}} \frac{\mathfrak{G}\left(\frac{3}{4} c z^{2}\right)}{\mathfrak{G}\left(\frac{3}{4} c z_{h}^{2}\right)} \mathfrak{G}\left(c z_{h}^{2}\right),
$$

where

$$
\mathfrak{G}(x)=x^{-1-\frac{1}{\nu}} \gamma\left(1+\frac{1}{\nu}, x\right)
$$

and the function $\mathfrak{G}(x)$ has the following expansion (see [56], p. 1377):

$$
\mathfrak{G}(x)=\sum_{n=0}^{\infty} \frac{(-1)^{n} x^{n}}{n !\left(1+n+\frac{1}{\nu}\right)} .
$$

Taking into account the first two terms of the expansion (2.40), we get

$$
g_{\text {appr }}(z)=1-\frac{z^{2+\frac{2}{\nu}}}{z_{h}^{2+\frac{2}{\nu}}}\left(\rho+Q z_{h}^{2}-Q z^{2}\right)
$$

where

$$
\begin{aligned}
\rho & =\frac{4(1+2 \nu)-3 c z^{2}(1+\nu)}{4(1+2 \nu)-3 c z_{h}^{2}(1+\nu)} \\
Q & =\frac{\mu^{2} c^{2} \nu z_{h}^{2+\frac{2}{\nu}} e^{\frac{c z_{h}^{2}}{2}}}{4\left(1-e^{\frac{c z_{h}^{2}}{4}}\right)^{2}\left(4(1+2 \nu)-3 c z_{h}^{2}(1+\nu)\right)}
\end{aligned}
$$

This expression can be recast into a form

$$
g_{\text {appr }}(z)=1-z^{2+\frac{2}{\nu}}\left(\left(\frac{\rho}{z_{h}}\right)^{2+\frac{2}{\nu}}+\frac{Q}{z_{h}^{2 / \nu}}\right)+Q \frac{z^{4+\frac{2}{\nu}}}{z_{h}^{2+\frac{2}{\nu}}}
$$

and after the redefinition $z_{h}=\tilde{z}_{h} \rho, Q=\tilde{Q} z_{h}^{2+\frac{2}{\nu}}$ it becomes

$$
g_{\text {appr }}(z)=1-z^{2+\frac{2}{\nu}}\left(\frac{1}{\tilde{z}_{h}^{2+\frac{2}{\nu}}}+\tilde{Q} \rho^{2} \tilde{z}_{h}^{2}\right)+\tilde{Q} z^{4+\frac{2}{\nu}} .
$$

The blackening function used in [4]

$$
g_{[2]}(z)=1-z^{2+\frac{2}{\nu}}\left(\frac{1}{z_{h}^{2+\frac{2}{\nu}}}+Q z_{h}^{2}\right) z^{2+\frac{2}{\nu}}+Q z^{4+\frac{2}{\nu}}
$$




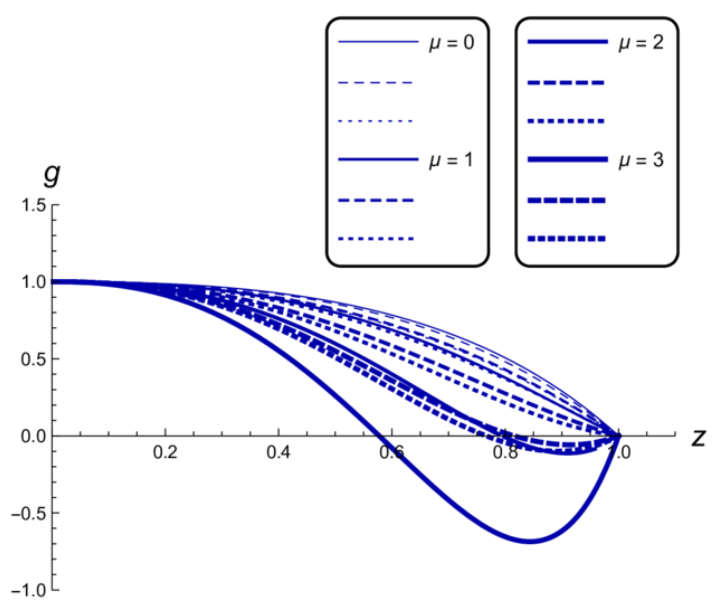

A

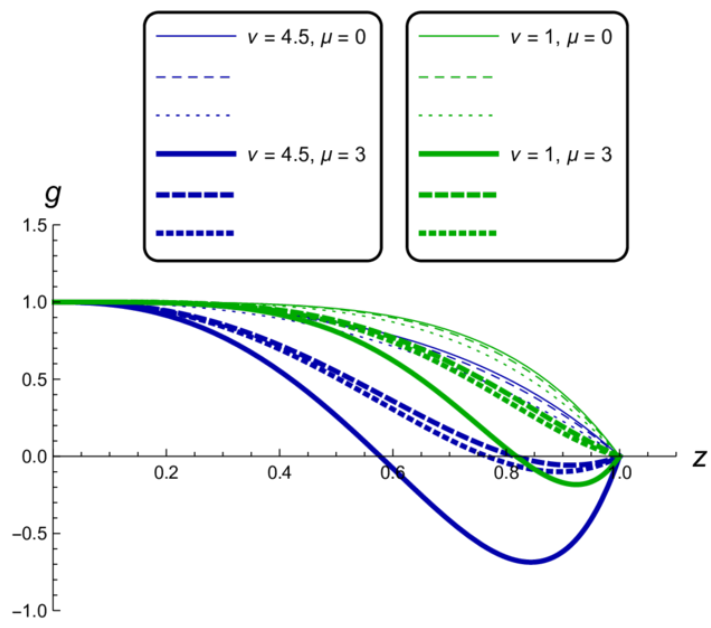

C

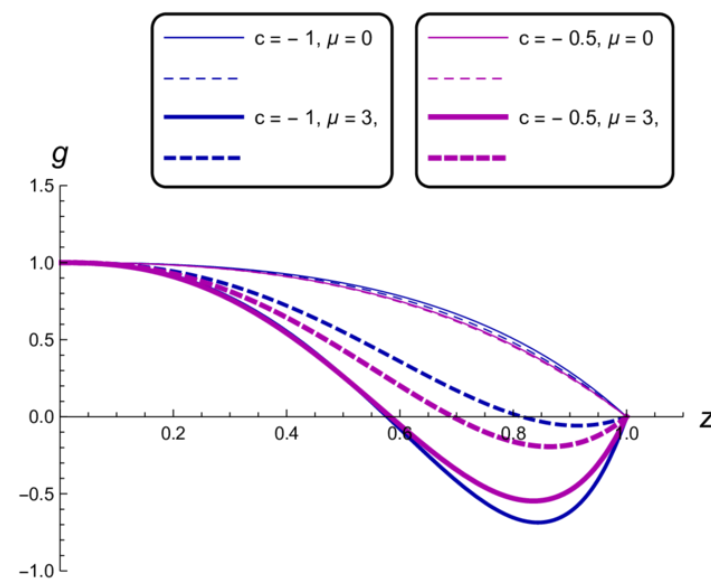

$\mathrm{B}$

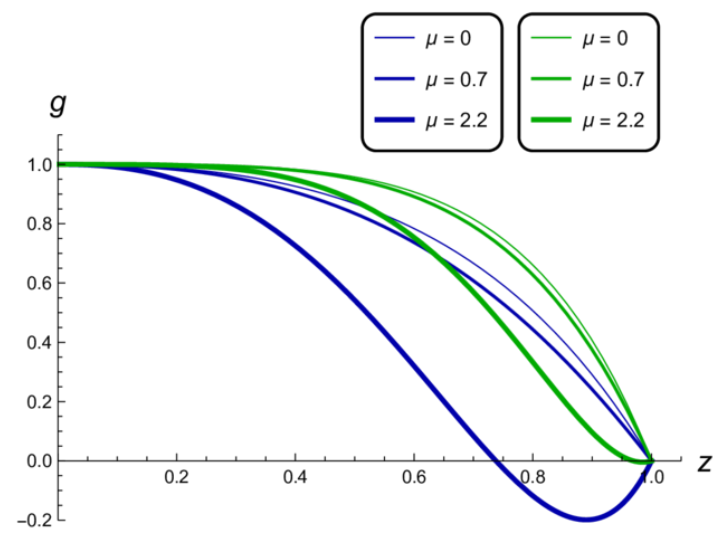

$\mathrm{D}$

Figure 1. Blackening functions $g(z)$ (solid lines), $g_{\text {approx }}(z)$ (same thickness dashed lines) and $g_{\text {approx },[2]}(z)$ (same thickness dotted lines) for $z_{h}=1, \nu=4.5, c=-1$ (blue lines), $\nu=4.5$, $c=-0.5$ (magenta lines), $\nu=1, c=-1$ (green lines) and different $\mu=0, \ldots, 3$.

is different by the factor $\rho^{2}$ in the second coefficient in front of $z^{2+\frac{2}{\nu}}$. Near the horizon this factor is approximately equal to 1 :

$$
\rho \approx 1-\frac{6 c z_{h}(1+\nu)}{4(1+2 \nu)-3 c z_{h}^{2}(1+\nu)}\left(z-z_{h}\right)+\mathcal{O}\left(\left(z-z_{h}\right)^{2}\right) .
$$

The behavior of the blackening function from the holographic coordinate $z$ till horizon is depicted on figure 1. The main feature is that the blackening function values decrease faster for larger chemical potential $\mu$ (figure 1.A) and for smaller warp factor coefficient $c$ (figure 1.B). The difference between the approximations (2.45) and (2.46) and the exact expression (2.37) is irregular and depends on the model parameters (figure 1.C). In the isotropic case the blackening function values are larger than in the anisotropic ones (figure 1.C and D). For $\mu$ close to zero it is the desreasing function of $z$ till the horizon, 
but for growing $\mu$ the local minimums and the second horizons small than the original ones appear. Changing the values of $c$ almost does not influence on the horizon position.

\subsubsection{Coupling function $f_{2}$}

We can substitute the expression for the blackening function (2.38) into (2.7), take into account

$$
g^{\prime}(z)=-2 \frac{z^{1+\frac{2}{\nu}}}{z_{h}^{2+\frac{2}{\nu}}} \frac{e^{-\frac{3 c z^{2}}{4}}}{\mathfrak{G}\left(\frac{3}{4} c z_{h}^{2}\right)}-\frac{\mu^{2} c z^{1+\frac{2}{\nu}} e^{-c z^{2}+\frac{c z_{h}^{2}}{2}}}{2\left(1-e^{\frac{c z_{h}^{2}}{4}}\right)^{2}}\left(1-e^{\frac{c z^{2}}{4}} \frac{\mathfrak{G}\left(c z_{h}^{2}\right)}{\mathfrak{G}\left(\frac{3}{4} c z_{h}^{2}\right)}\right)
$$

and get

$$
\begin{aligned}
& f_{2}(z)=\frac{\nu-1}{q^{2} \nu^{2}} z^{-\frac{4}{\nu}} e^{\frac{c z^{2}}{2}}\left[4(1+\nu)-3 c \nu z^{2}+4 \frac{z^{2+\frac{2}{\nu}}}{z_{h}^{2+\frac{2}{\nu}}}\left\{\frac{\nu e^{-\frac{3 c z^{2}}{4}}}{\mathfrak{G}\left(\frac{3}{4} c z_{h}^{2}\right)}-(1+\nu) \frac{\mathfrak{G}\left(\frac{3}{4} c z^{2}\right)}{\mathfrak{G}\left(\frac{3}{4} c z_{h}^{2}\right)} \mathfrak{F}\right.\right. \\
& \left.\left.+\frac{\mu^{2} c \nu z_{h}^{2+\frac{2}{\nu}} e^{-c z^{2}+\frac{c z_{h}^{2}}{2}}}{4\left(1-e^{\frac{c z_{h}^{2}}{4}}\right)^{2}}\left(1-e^{\frac{c z^{2}}{4}} \frac{\mathfrak{G}\left(c z_{h}^{2}\right)}{\mathfrak{G}\left(\frac{3}{4} c z_{h}^{2}\right)}\right)\right\}+3 c \nu \frac{z^{4+\frac{2}{\nu}}}{z_{h}^{2+\frac{2}{\nu}}} \frac{\mathfrak{G}\left(\frac{3}{4} c z^{2}\right)}{\mathfrak{G}\left(\frac{3}{4} c z_{h}^{2}\right)} \mathfrak{F}\right],
\end{aligned}
$$

where

$$
\mathfrak{F}=1-\frac{\mu^{2} c z_{h}^{2+\frac{2}{\nu}} e^{\frac{c z_{h}^{2}}{2}}}{4\left(1-e^{\frac{c z_{h}^{2}}{4}}\right)^{2}}\left(\mathfrak{G}\left(c z_{h}^{2}\right)-\mathfrak{G}\left(c z^{2}\right) \frac{\mathfrak{G}\left(\frac{3}{4} c z_{h}^{2}\right)}{\mathfrak{G}\left(\frac{3}{4} c z^{2}\right)}\right) .
$$

At the horizon

$$
f_{2}\left(z_{h}\right)=4 \frac{\nu-1}{q^{2} \nu} z^{-\frac{4}{\nu}}\left\{\frac{e^{-\frac{c z_{h}^{2}}{4}}}{\mathfrak{G}\left(\frac{3}{4} c z_{h}^{2}\right)}+\frac{\mu^{2} c z_{h}^{2+\frac{2}{\nu}}}{4\left(1-e^{\frac{c z_{h}^{2}}{4}}\right)^{2}}\left(1-e^{\frac{c z_{h}^{2}}{4}} \frac{\mathfrak{G}\left(c z_{h}^{2}\right)}{\mathfrak{G}\left(\frac{3}{4} c z_{h}^{2}\right)}\right)\right\}
$$

Using the first two terms of the expansion (2.40) on $z$ and $z_{h}$

$$
\begin{aligned}
\mathfrak{G}\left(c z_{(h)}^{2}\right) & =\frac{\nu}{\nu+1}-\frac{c \nu z_{(h)}^{2}}{2 \nu+1}, \\
\mathfrak{G}\left(\frac{3}{4} c z_{(h)}^{2}\right) & =\left(\frac{3}{4}\right)^{1+\frac{1}{\nu}}\left(\frac{\nu}{\nu+1}-\frac{3}{4} \frac{c \nu z_{(h)}^{2}}{2 \nu+1}\right),
\end{aligned}
$$




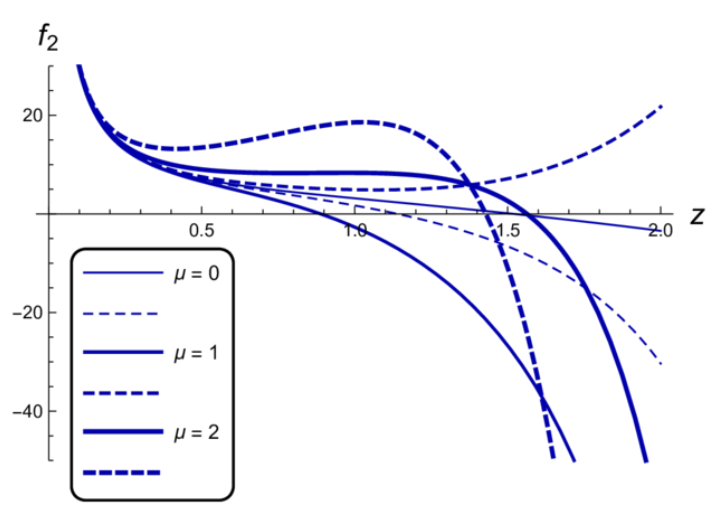

A

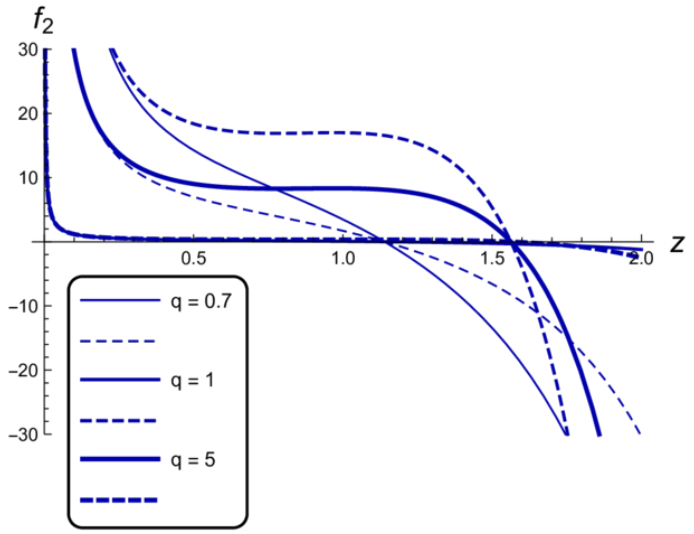

B

Figure 2. Coupling function $f_{2}(z)$ (solid lines) and $f_{2}$ approx (same thickness dashed lines) for $z_{h}=1, \nu=4.5, c=-1, q=1, \mu=0,1,5$ (A) and $\mu=1, q=0.7,1,5$ (B).

we have the following expansion of coupling factor $f_{2}$ :

$$
\begin{aligned}
f_{2 \text { approx }}(z)= & \frac{\nu-1}{q^{2} \nu^{2}} z^{-\frac{4}{\nu}} e^{\frac{c z^{2}}{2}}\left[4(1+\nu)-3 c \nu z^{2}\right. \\
+ & \frac{z^{2+\frac{2}{\nu}}}{z_{h}^{2+\frac{2}{\nu}}}\left\{\frac{16(1+\nu)(1+2 \nu)}{3 c z_{h}^{2}(1+\nu)-4(1+2 \nu)}\left(1-\frac{4^{1+\frac{1}{\nu}}}{3^{1+\frac{1}{\nu}}} e^{-\frac{3 c z^{2}}{4}}\right)\right. \\
& \left.-\frac{\mu^{2} c \nu z_{h}^{2+\frac{2}{\nu}} e^{\frac{c z_{h}^{2}}{2}}}{\left(1-e^{\frac{c z_{h}^{2}}{4}}\right)^{2}}\left(1-e^{-c z^{2}}-4 \frac{c z_{h}^{2}(1+\nu)-(1+2 \nu)}{3 c z_{h}^{2}(1+\nu)-4(1+2 \nu)}\left(1-\frac{4^{1+\frac{1}{\nu}}}{3^{1+\frac{1}{\nu}}} e^{-\frac{3 c z^{2}}{4}}\right)\right)\right\} \\
& -\frac{z^{4+\frac{2}{\nu}}}{z_{h}^{2+\frac{2}{\nu}}}\left\{\frac{12 c(1+3 \nu(1+\nu))}{3 c z_{h}^{2}(1+\nu)-4(1+2 \nu)}+\frac{\mu^{2} c^{2} \nu z_{h}^{2+\frac{2}{\nu}} e^{\frac{c z_{h}^{2}}{2}}}{4\left(1-e^{\frac{c z_{h}^{2}}{4}}\right)^{2}} \frac{3 c \nu z_{h}^{2}+4(1+2 \nu)}{3 c z_{h}^{2}(1+\nu)-4(1+2 \nu)}\right\} \\
+ & \left.\frac{z^{6+\frac{2}{\nu}}}{z_{h}^{2+\frac{2}{\nu}}} \frac{9 c^{2} \nu(1+\nu)}{3 c z_{h}^{2}(1+\nu)-4(1+2 \nu)}\left(1+\frac{\mu^{2} c \nu z_{h}^{2+\frac{2}{\nu}} e^{\frac{c z_{h}^{2}}{2}}}{12\left(1-e^{\frac{c z_{h}^{2}}{4}}\right)^{2}(1+\nu)}\right)\right]
\end{aligned}
$$

and at the horizon

$$
\begin{aligned}
f_{2 \text { approx }}\left(z_{h}\right)= & \frac{1-\nu}{q^{2} \nu^{2}} z_{h}^{-\frac{4}{\nu}} e^{-\frac{c z_{h}^{2}}{4}}\left\{\frac{4^{3+\frac{1}{\nu}}}{3^{1+\frac{1}{\nu}}} \frac{(1+\nu)(1+2 \nu)}{3 c z_{h}^{2}(1+\nu)-4(1+2 \nu)}\right. \\
& \left.-\frac{\mu^{2} c \nu z_{h}^{2+\frac{2}{\nu}} e^{\frac{c z_{h}^{2}}{4}}}{\left(1-e^{\frac{c z_{h}^{2}}{4}}\right)^{2}}\left(1-\frac{4^{2+\frac{1}{\nu}}}{3^{1+\frac{1}{\nu}}} \frac{c z_{h}^{2}(1+\nu)-(1+2 \nu)}{3 c z_{h}^{2}(1+\nu)-4(1+2 \nu)} e^{\frac{c z^{2}}{4}}\right)\right\} .
\end{aligned}
$$

If we substitute $g_{\text {appr }}(z)$ into $f_{2}$, the result coincides with (2.54) (figure 2). 


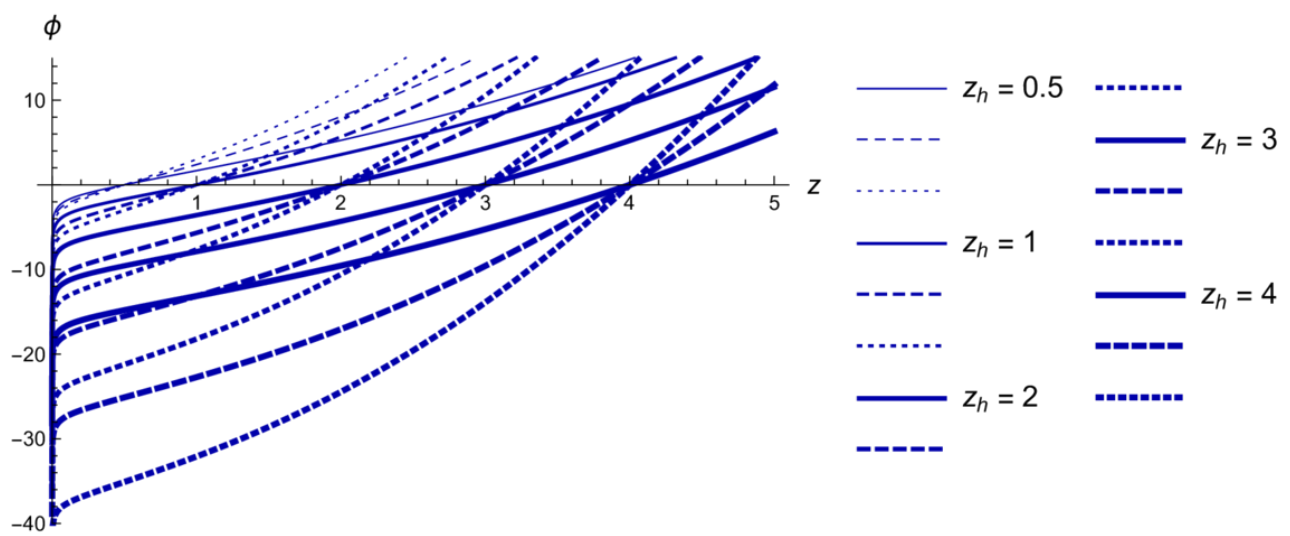

Figure 3. Scalar field $\phi(z)$ for $\nu=4.5, c=-1$ (solid lines), $c=-2$ (dashed lines) and $c=-3$ (dotted lines) and different $z_{h}$.

\subsubsection{Scalar field}

Substitution of (2.31) into (2.6) leads to

$$
\phi^{\prime}=\frac{1}{\nu z} \sqrt{\frac{3}{2} \nu^{2} c^{2} z^{4}-9 \nu^{2} c z^{2}+4 \nu-4}
$$

Here we should take into account, that the radicand in (2.56) shouldn't be negative. Therefore we have different cases of parameter $c$ value.

- $c<\mathbf{0}$. For $c<0$ this requirement is fullfiled without any restrictions and for the scalar field we have (figure 3)

$$
\begin{aligned}
\phi= & \frac{1}{2 \sqrt{2} \nu}\left\{\sqrt{3 c^{2} \nu^{2} z^{4}-18 c \nu^{2} z^{2}+8(\nu-1)}-\sqrt{3 c^{2} \nu^{2} z_{h}^{4}-18 c \nu^{2} z_{h}^{2}+8(\nu-1)}\right. \\
& +2 \sqrt{2(\nu-1)} \ln \left(\frac{z^{2}}{z_{h}^{2}}\right)-3 \sqrt{3} \nu \ln \left(\frac{\sqrt{3 c^{2} \nu^{2} z^{4}-18 c \nu^{2} z^{2}+8(\nu-1)}-\sqrt{3} \nu\left(3-c z^{2}\right)}{\sqrt{3 c^{2} \nu^{2} z_{h}^{4}-18 c \nu^{2} z_{h}^{2}+8(\nu-1)}-\sqrt{3} \nu\left(3-c z_{h}^{2}\right)}\right) \\
& \left.-2 \sqrt{2(\nu-1)} \ln \left(\frac{9 c \nu^{2} z^{2}-8(\nu-1)-\sqrt{2(\nu-1)} \sqrt{3 c^{2} \nu^{2} z^{4}-18 c \nu^{2} z^{2}+8(\nu-1)}}{9 c \nu^{2} z_{h}^{2}-8(\nu-1)-\sqrt{2(\nu-1)} \sqrt{3 c^{2} \nu^{2} z_{h}^{4}-18 c \nu^{2} z_{h}^{2}+8(\nu-1)}}\right)\right\} .
\end{aligned}
$$

For small $z$ the scalar field can be approximated as

$$
\phi \sim-k\left(z_{h}, \nu, c\right)+\frac{2 \sqrt{\nu-1}}{\nu} \log \left(\frac{z}{z_{h}}\right)
$$

and for large $z$

$$
\phi \sim \frac{|c|}{2} \sqrt{\frac{3}{2}} z^{2} .
$$


- $\boldsymbol{c}>\mathbf{0}$. For $c>0$ expression (2.56) can be parametrized as

$$
\phi^{\prime}=\frac{c}{z} \sqrt{\frac{3}{2}\left(\alpha^{2}-z^{2}\right)\left(\beta^{2}-z^{2}\right)}
$$

where

$$
\alpha^{2} \beta^{2}=\frac{8(\nu-1)}{3 \nu^{2} c^{2}}, \quad \alpha^{2}+\beta^{2}=\frac{6}{c}
$$

and

$$
\alpha=\sqrt{\frac{3}{c}-\frac{1}{c} \sqrt{9-\frac{8(\nu-1)}{3 \nu^{2}}}}, \quad \beta=\sqrt{\frac{3}{c}+\frac{1}{c} \sqrt{9-\frac{8(\nu-1)}{3 \nu^{2}}}} .
$$

Note, that we can get a real solution only for

$$
\begin{aligned}
\text { i) } & \alpha^{2}-z^{2}>0, \quad \beta^{2}-z^{2}>0, \\
\text { ii) } & \alpha^{2}-z^{2}<0, \quad \beta^{2}-z^{2}<0 .
\end{aligned}
$$

Integrating (2.60) we obtain

$$
\begin{aligned}
\phi(z)= & \sqrt{\frac{3}{8}} c\left\{\sqrt{\left(\alpha^{2}-z^{2}\right)\left(\beta^{2}-z^{2}\right)}-\sqrt{\left(\alpha^{2}-z_{h}^{2}\right)\left(\beta^{2}-z_{h}^{2}\right)}+\alpha \beta \ln \left(\frac{z^{2}}{z_{h}^{2}}\right)\right. \\
& -\frac{\alpha^{2}+\beta^{2}}{2} \ln \left(\frac{\sqrt{\left(\alpha^{2}-z^{2}\right)\left(\beta^{2}-z^{2}\right)}+z^{2}-\frac{\alpha^{2}+\beta^{2}}{2}}{\sqrt{\left(\alpha^{2}-z_{h}^{2}\right)\left(\beta^{2}-z_{h}^{2}\right)}+z_{h}^{2}-\frac{\alpha^{2}+\beta^{2}}{2}}\right) \\
& \left.-\alpha \beta \ln \left(\frac{\alpha^{2} \beta^{2}-\frac{\alpha^{2}+\beta^{2}}{2} z^{2}+\alpha \beta \sqrt{\left(\alpha^{2}-z^{2}\right)\left(\beta^{2}-z^{2}\right)}}{\alpha^{2} \beta^{2}-\frac{\alpha^{2}+\beta^{2}}{2} z_{h}^{2}+\alpha \beta \sqrt{\left(\alpha^{2}-z_{h}^{2}\right)\left(\beta^{2}-z_{h}^{2}\right)}}\right)\right\}
\end{aligned}
$$

We see that the solution (B.21) becomes complex for $\alpha<z<\beta$. It leads to an instability region for the scalar field (figure 4).

- $\boldsymbol{c}=\mathbf{0}$. For $c=0$ we get (figure 5)

$$
\begin{aligned}
\phi^{\prime} & =\frac{2 \sqrt{\nu-1}}{\nu z} \\
\phi & =\frac{2 \sqrt{\nu-1}}{\nu} \ln \left(\frac{z}{z_{h}}\right) .
\end{aligned}
$$



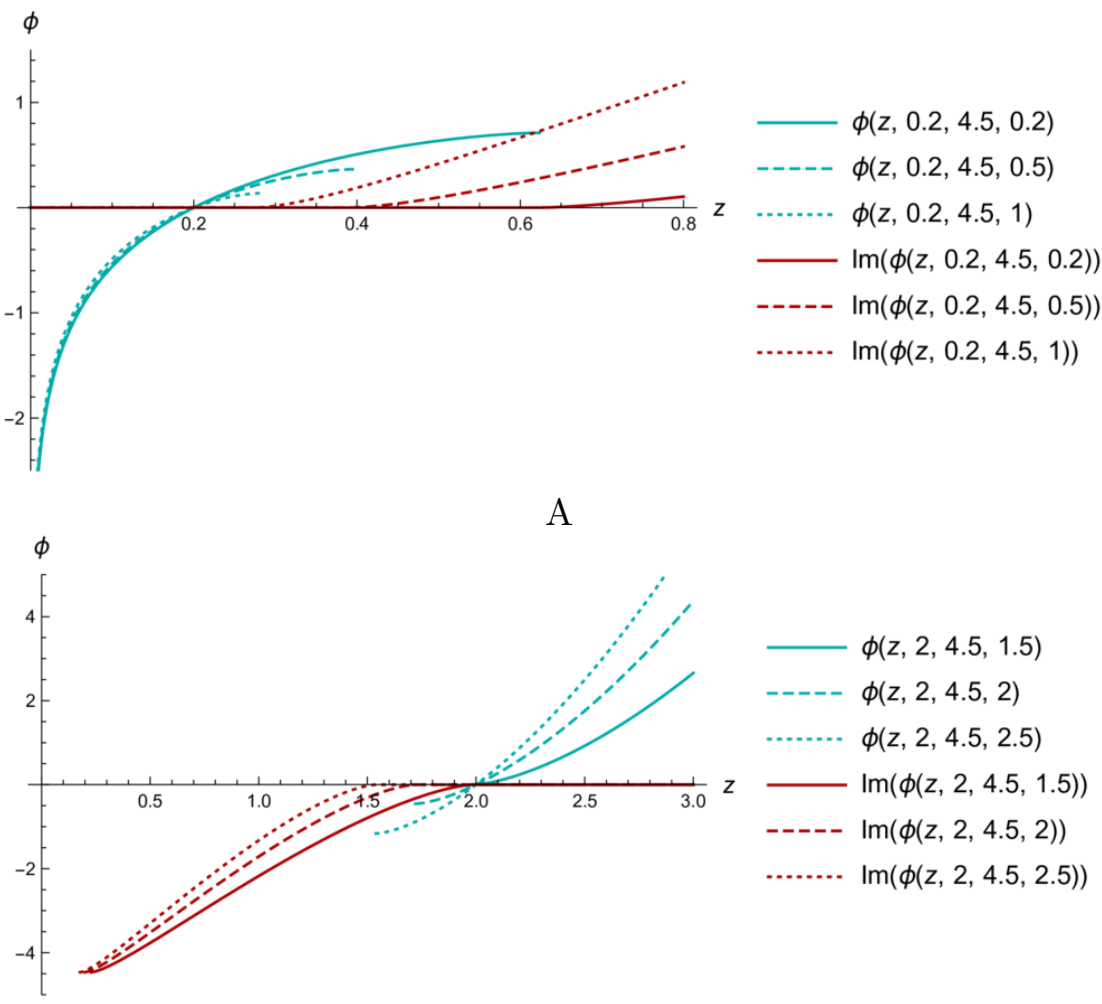

$\mathrm{B}$

Figure 4. Scalar field $\phi\left(z, z_{h}, \nu, c\right)$ and its imaginary part for $\nu=4.5$, different positive $c, z_{h}=0.2$ (A) and $z_{h}=2(\mathrm{~B})$.

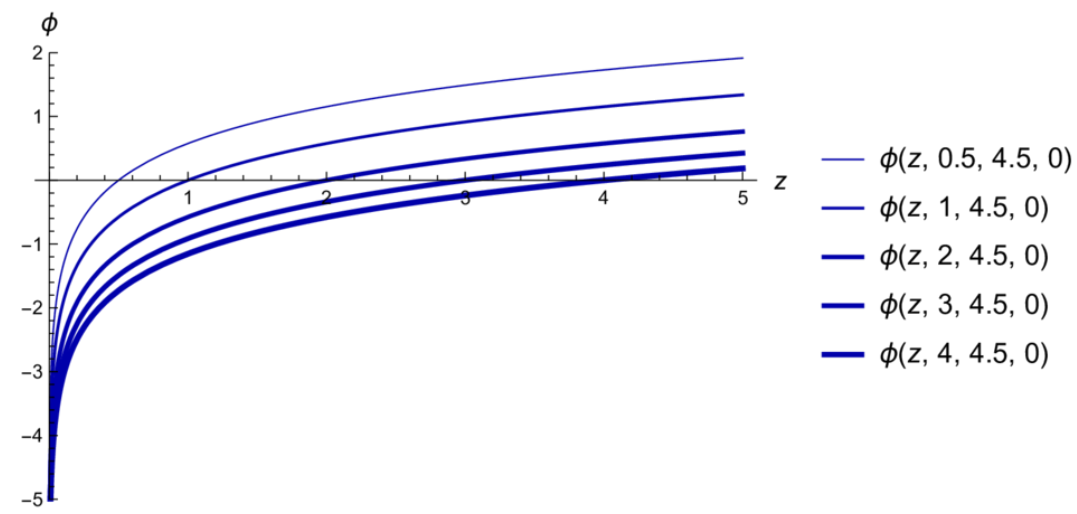

Figure 5. Scalar field $\phi\left(z, z_{h}, \nu, c\right)$ for $\nu=4.5, c=0$ and different $z_{h}$. 


\subsubsection{Scalar potential}

From equation (2.8) we get the expression for the scalar potential $V$ as a function of $z$ :

$$
\begin{aligned}
V(z)= & \frac{e^{-\frac{c z^{2}}{2}}}{8 \nu^{2}}\left\{-16\left(2 \nu^{2}+3 \nu+1\right)+12 c \nu z^{2}(2 \nu+3)+16\left(2 \nu^{2}+3 \nu+1\right) z^{2+\frac{2}{\nu}} \mathfrak{V}\right. \\
& -16 \nu(2 \nu+1) \frac{z^{2+\frac{2}{\nu}}}{z_{h}^{2+\frac{2}{\nu}}} e^{-c z^{2}}\left[\frac{e^{\frac{c z^{2}}{4}}}{\mathfrak{G}\left(\frac{3}{4} c z_{h}^{2}\right)}+\frac{\mu^{2} c z_{h}^{2+\frac{2}{\nu}} e^{\frac{c z_{h}^{2}}{2}}}{4\left(1-e^{\frac{c z_{h}^{2}}{4}}\right)^{2}}\left(1-e^{\frac{c z^{2}}{4}} \frac{\mathfrak{G}\left(c z_{h}^{2}\right)}{\mathfrak{G}\left(\frac{3}{4} c z_{h}^{2}\right)}\right)\right] \\
& -18 c^{2} \nu^{2} z^{4}-12 c \nu(2 \nu+3) z^{4+\frac{2}{\nu}} \mathfrak{V} \\
& -\nu \frac{z^{4+\frac{2}{\nu}}}{z_{h}^{2+\frac{2}{\nu}}} e^{-c z^{2}}\left[\frac{-24 c \nu e^{\frac{c z^{2}}{4}}}{\mathfrak{G}\left(\frac{3}{4} c z_{h}^{2}\right)}-\frac{5 \mu^{2} c^{2} \nu z_{h}^{2+\frac{2}{\nu}} e^{\frac{c z_{h}^{2}}{2}}}{\left(1-e^{\frac{c z_{h}^{2}}{4}}\right)^{2}}\left(1-\frac{6}{5} e^{\frac{c z^{2}}{4}} \frac{\mathfrak{G}\left(c z_{h}^{2}\right)}{\mathfrak{G}\left(\frac{3}{4} c z_{h}^{2}\right)}\right)\right] \\
& \left.+18 c^{2} \nu^{2} z^{6+\frac{2}{\nu}} \mathfrak{V}\right\},
\end{aligned}
$$

where

$$
\mathfrak{V}=\frac{1}{z_{h}^{2+\frac{2}{\nu}}} \frac{\mathfrak{G}\left(\frac{3}{4} c z^{2}\right)}{\mathfrak{G}\left(\frac{3}{4} c z_{h}^{2}\right)}+\frac{\mu^{2} c e^{\frac{c z_{h}^{2}}{2}}}{4\left(1-e^{\frac{c z_{h}^{2}}{4}}\right)^{2}}\left(\mathfrak{G}\left(c z^{2}\right)-\mathfrak{G}\left(c z_{h}^{2}\right) \frac{\mathfrak{G}\left(\frac{3}{4} c z^{2}\right)}{\mathfrak{G}\left(\frac{3}{4} c z_{h}^{2}\right)}\right)
$$

The dependence $V(\phi)$ can't be expressed explicitly due to nontrivial behavior of $\phi(z)$ (2.57), but it can be displayed graphically (figure 6). For $c=-1$ it can be approximated by a sum of two exponents and a negative constant:

$$
V_{2 E A}(\phi, \mu, \nu)=V_{0}(\nu)-C_{7}(\mu, \nu) e^{K_{1}(\nu) \phi}+C_{8}(\mu, \nu) e^{K_{2}(\nu) \phi} .
$$

The best fit is given by

$$
V_{0}(4.5)=-0.5778, \quad K_{1}(4.5)=0.7897, \quad K_{2}(4.5)=2.0995
$$

with the coefficients depending on the chemical potential $\mu$ (figure 6.A and B):

$$
C_{7}(\mu, 4.5)=23.0779+2.4236 \mu^{2}, \quad C_{8}(\mu, 4.5)=0.0575+4.9919 \mu^{2} .
$$

In isotropic case (figure 6.C and D) the approximation constants are:

$$
\begin{aligned}
& V_{0}(1)=-10.8689, \quad K_{1}(1)=1.0852, \quad K_{2}(1)=2.4103, \\
& C_{7}(\mu, 1)=27.2825+4.3749 \mu^{2}, \quad C_{8}(\mu, 1)=0.0031+5.03093 \mu^{2} .
\end{aligned}
$$

Note, that in [57] an explicit isotropic solution for the dilaton potential as a sum of two exponents and zero chemical potential has been constructed. It would be interesting to generalize this construction to the anisotropic and non-zero chemical potential cases. 


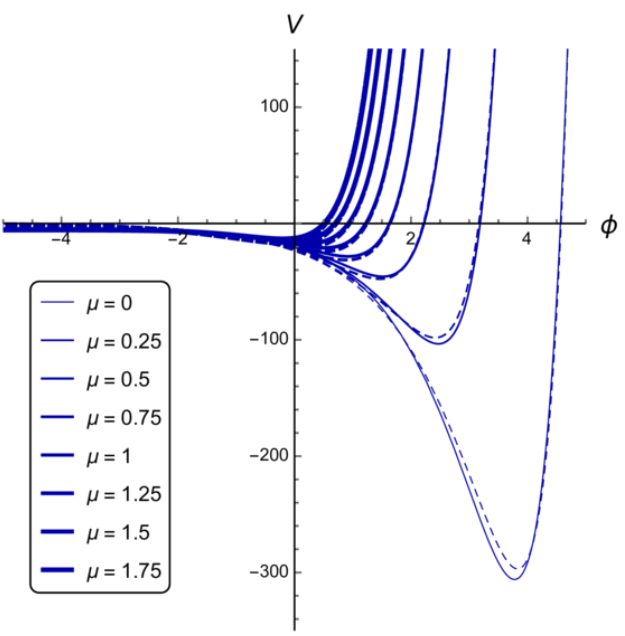

A

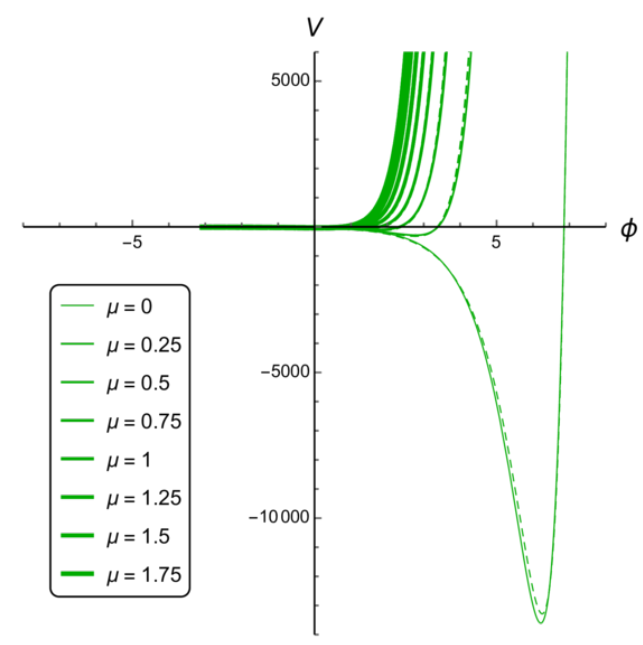

$\mathrm{C}$

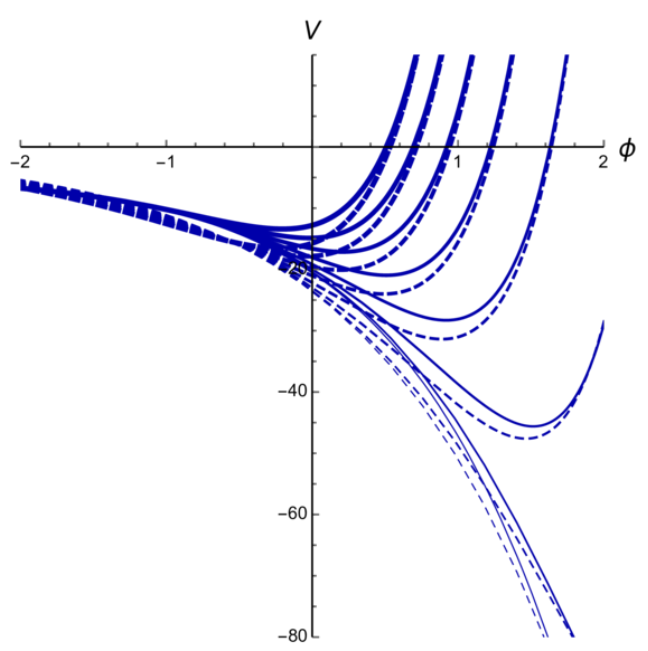

$\mathrm{B}$

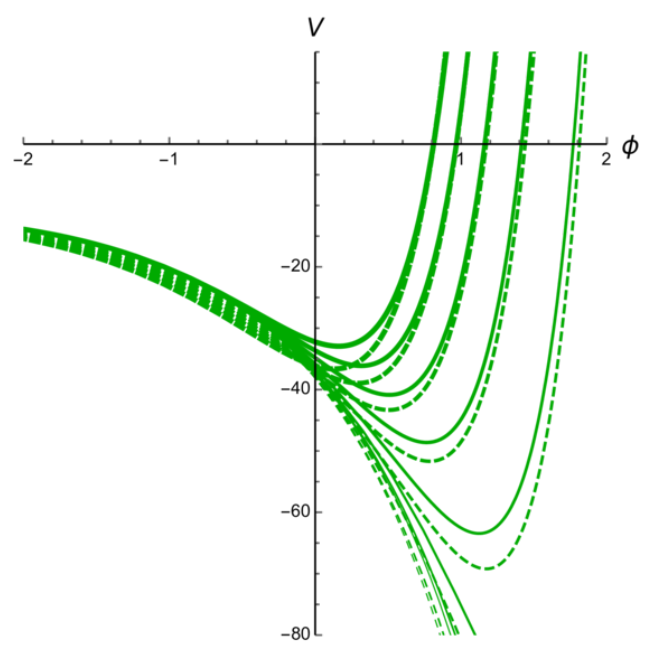

$\mathrm{D}$

Figure 6. Scalar field potential $V(\phi)$ (solid lines) and its approximation (dashed lines) as a sum of two exponents and a constant for $z_{h}=1, c=-1$ and different $\mu$ in anisotropic, $\nu=4.5,(\mathrm{~A})$ and isotropic (C) cases. The right plots (B) and (D) are zoom of the corresponding left ones.

The behavior of $V(\phi)$ for positive warp factor coefficient is quite different. Let us recall that for $c>0$ the scalar field $\phi$ becomes complex under horizon (figure 7 ). The function $V(\operatorname{Re}(\phi))$ doesn't display visible dependence on chemical potential (figure 8.A) and the function $V(|\phi|)$ stops to depend on $\mu$ rather soon (figure 8.B).

\subsection{Scalar invariants}

For completeness we present here the dependence of the scalar invariants $R, R^{2}=R_{\mu \nu} R^{\mu \nu}$ and $K=R_{\mu \nu \rho \sigma} R^{\mu \nu \rho \sigma}$ on the parameter $\mu$ for the unit horizon and negative warp factor coefficient $c$. All the invariants are smooth inside the black hole and start to diverge for $z>z_{h}$ (figure 9, 10). In isotropic case it happens earlier, means for smaller $z$, than is anisotropic one. Thus the horizons of the blackening function, depicted on figure 1, are regular. 


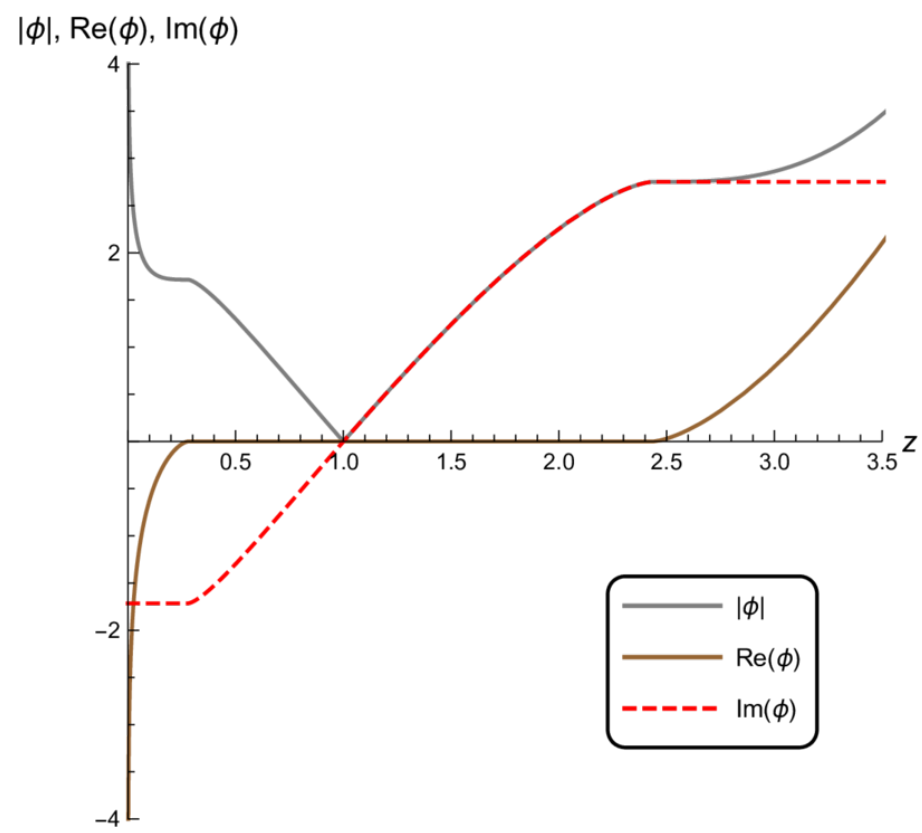

Figure 7. Module, real and imaginary parts of the scalar field $\phi$ for $\nu=4.5, z_{h}=1, c=1$.

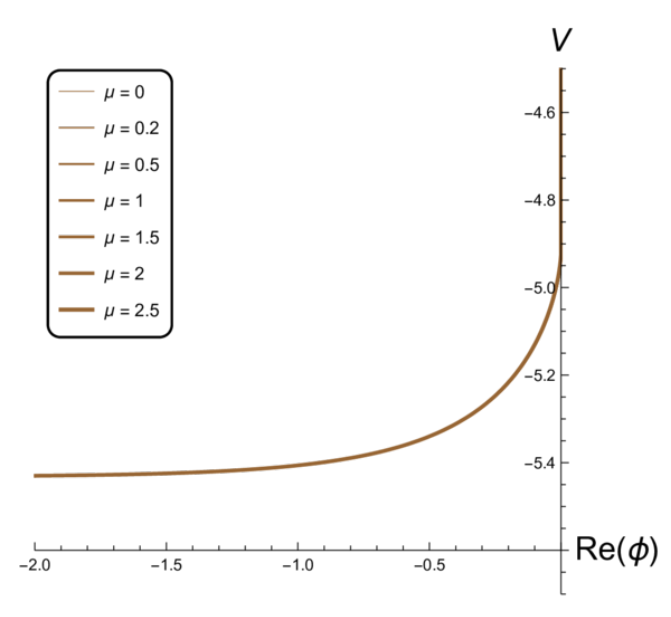

A

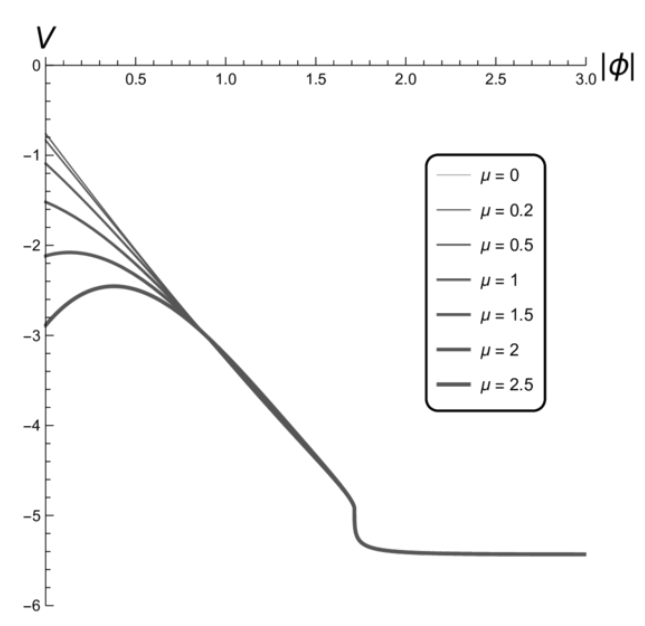

$\mathrm{B}$

Figure 8. Scalar potential $V(\operatorname{Re}(\phi))(\mathrm{A})$ and $V(|\phi|)(\mathrm{B})$ for $\nu=4.5, z_{h}=1, c=1$ and different $\mu$ under horizon, i.e. $0<z<1$.

\subsection{RG flow}

Our background is an anisotropic analog of the background used in the improved holographic QCD model [22]. The holographic coordinate $z$ corresponds to the 4D RG scale. According to holographic dictionary one identifies the $4 \mathrm{D}$ energy scale $E$ with the metric scalar factor, i.e. $E=E_{0} L \sqrt{b(z)} / z \equiv E_{0} L B(z)$, in what follows we put $E_{0} L=1$. The running 't Hooft coupling $\lambda_{t}$ is identified with the string coupling $\lambda=e^{\phi}$ up to a factor, 


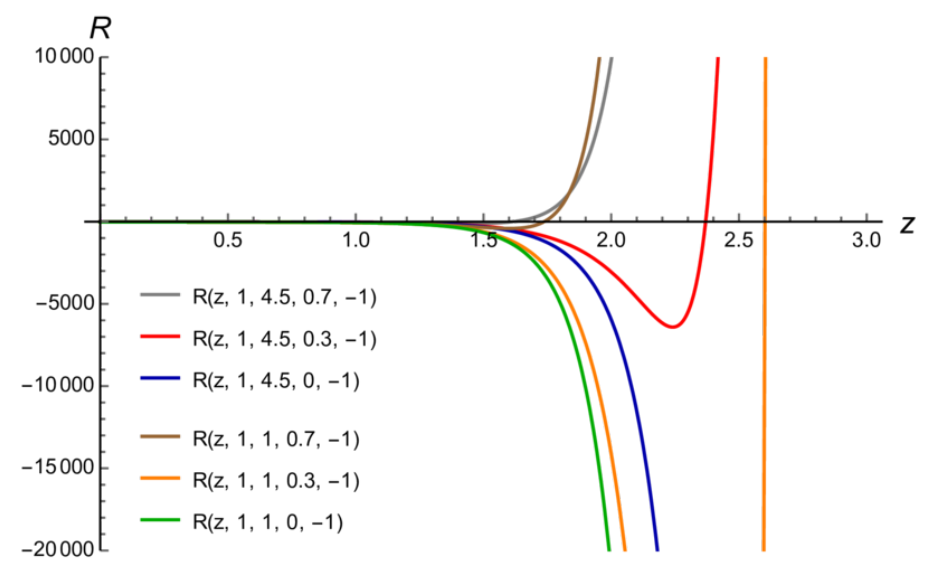

Figure 9. Ricci scalar $R(z)$ for $z_{h}=1, c=-1$ in isotropic, $\nu=1$, and anisotropic, $\nu=4.5$, cases for different $\mu$.

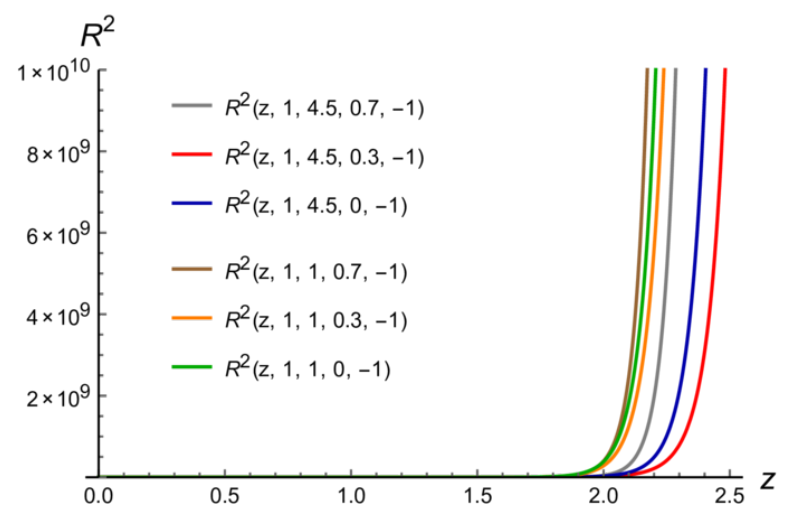

A

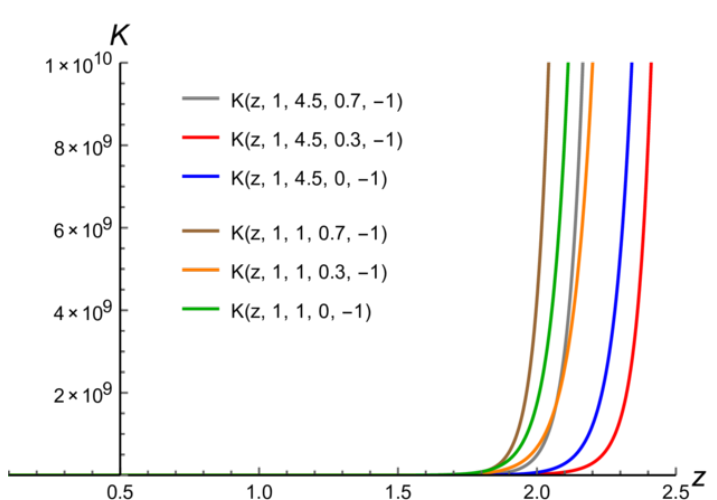

$\mathrm{B}$

Figure 10. Ricci $R^{2}(z)$ and Riemann $K(z)$ invariants for $z_{h}=1, c=-1$ in isotropic, $\nu=1$, and anisotropic, $\nu=4.5$, cases for different $\mu$.

$\lambda=\kappa \lambda_{t}$. In figure 11.A we show the dependence of coupling constant on the energy parameter for isotropic and anisotpopic cases. We see that the running coupling constant decreases from the IR region to the UV region. This behavior reproduces our expectations of the running coupling view in a nonperturbative QCD. Note, that the anisotropic case does not differ much from the isotropic one. The difference becomes more essential for small $z_{h}$.

The $\beta$-function in terms of the background is defined as $[5,58]$

$$
\beta(\lambda)=\frac{d \lambda}{d \log E}=\lambda \frac{d \phi}{d \log B}, \quad B=\frac{\sqrt{b(z)}}{z} .
$$

Introducing the function $X$, related with the $\beta$-function as

$$
X(\phi)=\frac{\beta(\lambda)}{3 \lambda}=\frac{B}{B^{\prime}} \frac{\phi^{\prime}}{3}
$$




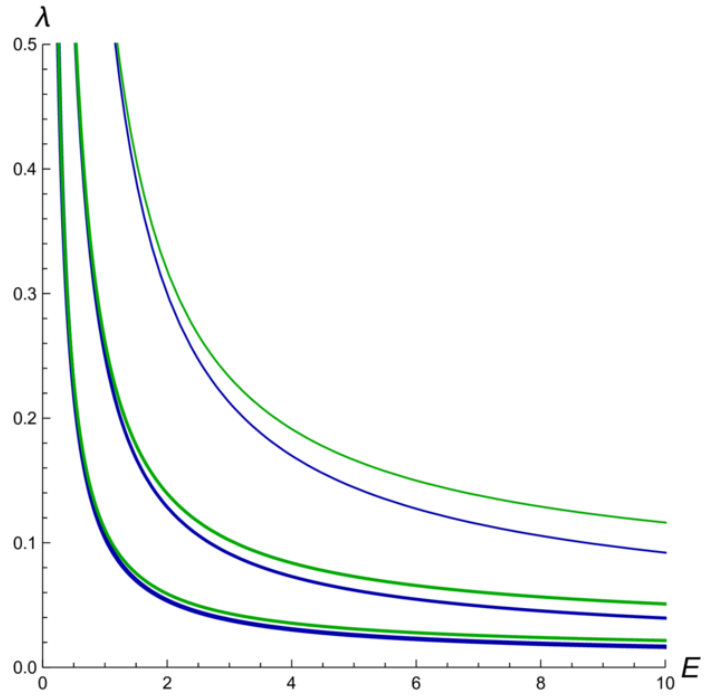

A

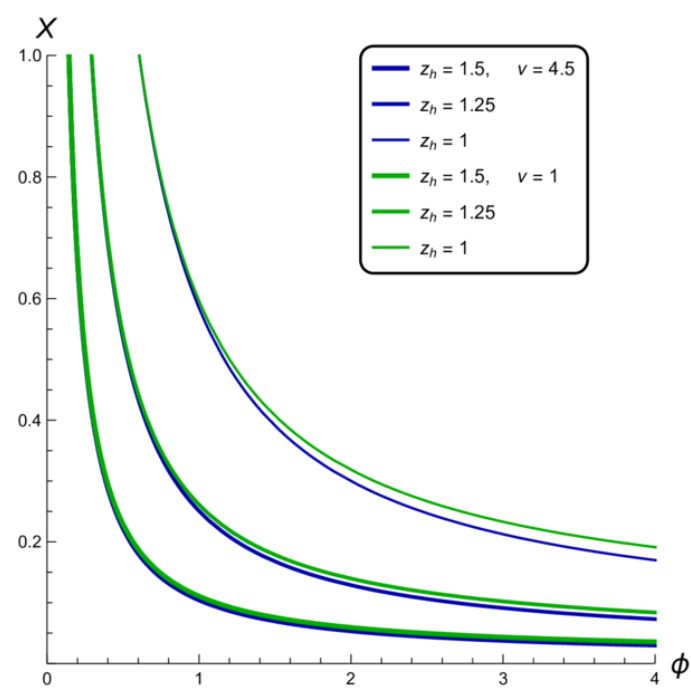

$\mathrm{B}$

Figure 11. The running coupling as function of the energy scale (A) and the RG flow (B) in isotropic and anisotropic $\nu=4.5$ case; the plot legends are the same for both panels.

the function $Y$, related with the blackening function as

$$
Y(\phi)=\frac{1}{4} \frac{g^{\prime}}{g} \frac{B}{B^{\prime}},
$$

and the function $H$, related with the vector field provided by non-zero chemical potential as

$$
H(\phi)=\frac{A_{t}^{\prime}}{B}
$$

one can check that in the isotropic case due to E.O.M. these quantities satisfy the first order differential equations

$$
\begin{aligned}
\frac{d X}{d \phi} & =-\frac{4}{3}\left(1-\frac{3}{8} X^{2}+Y\right)\left(1+\frac{1}{X} \frac{2 \partial_{\phi} V-H^{2} \partial_{\phi} f_{1}}{2 V+H^{2} f_{1}}\right), \\
\frac{d Y}{d \phi} & =-\frac{4 Y}{3 X}\left(1-\frac{3}{8} X^{2}+Y\right)\left(1+\frac{3}{2 Y} \frac{H^{2} f_{1}}{2 V+H^{2} f_{1}}\right), \\
\frac{d H}{d \phi} & =-\left(\frac{1}{X}+\frac{\partial_{\phi} f_{1}}{f_{1}}\right) H,
\end{aligned}
$$

where $\partial_{\phi}=\partial / \partial \phi$. The anisotropic case is more subtle and will be the subject of a forthcoming paper.

Figure 12 shows the $X$-flow in the anisotropic case for $\nu=4.5$ and different $z_{h}$ in the $(\phi, X)$ - and in the $(\lambda, X)$-planes. The function $X(\phi)$ decreases starting from a constant value up to a local minimum as the argument grows and is shifted to the right for larger horizon. The function $X(\lambda)$ for $z_{h}<4$ smoothly decreases with increasing $\lambda$ and for $z_{h}>4$ the dependence is more complicated.

In figure 13 the behavior of functions $\beta(\lambda)$ in anisotropic, $\nu=4.5$, (figure 13.A) and isotropic (figure 13.C) cases and the corresponding potentials $V(\phi)$ (figure 13.B and D) for 


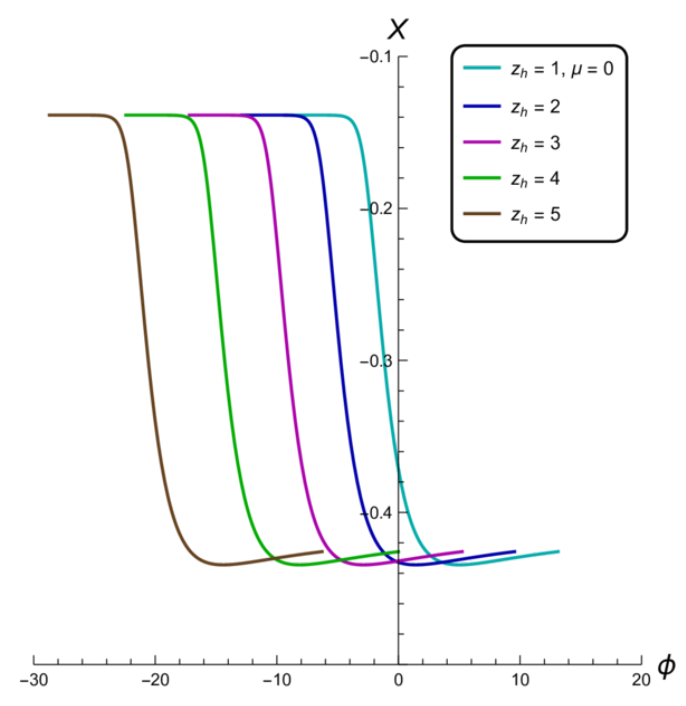

$\mathrm{A}$

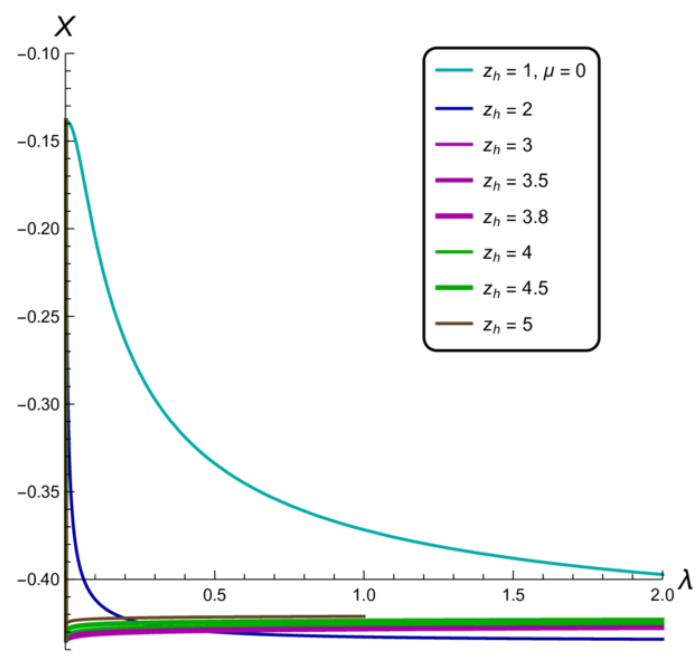

B

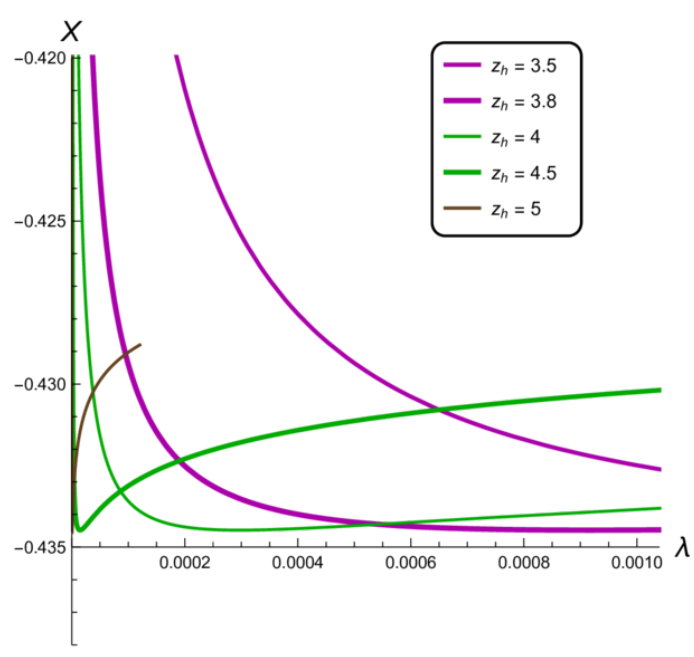

C

Figure 12. The RG flows for anisotropic case $\nu=4.5$ and different $z_{h}$ in the $(\phi, X)$-plane (A), in the $(\lambda, X)$-plane $(\mathrm{B})$ and in a small part of $(\mathrm{B})$ near the origin $(\mathrm{C})$.

the same horizon values are shown. We see, that for both cases $\beta(\lambda)<0$ in an agreement with the asymptotical freedom. For larger $z_{h}$ both functions display more non-linearity and decrease faster with the argument grow. This tendency is peculiar either for the isotropic or the anisotropic case. The only special difference is the non-zero value of $\beta(0)$ for $\nu=1$. We expect that changing the form of $P(z)$ we can, as in the isotropic case $[5,40,58]$, to recover the first orders expression of the perturbative $\beta$-function.

In figure 14 we show the dependence of the $\beta$-function on $H$ for the isotropic and anisotropic $(\nu=4.5)$ cases. We see that $\beta$ is the increasing function of $H$, approximately linear for large negative argument values and displaying its non-linearity near zero. The function values are visibly larger for larger chemical potential, while the anisotropy does not change this picture much. 


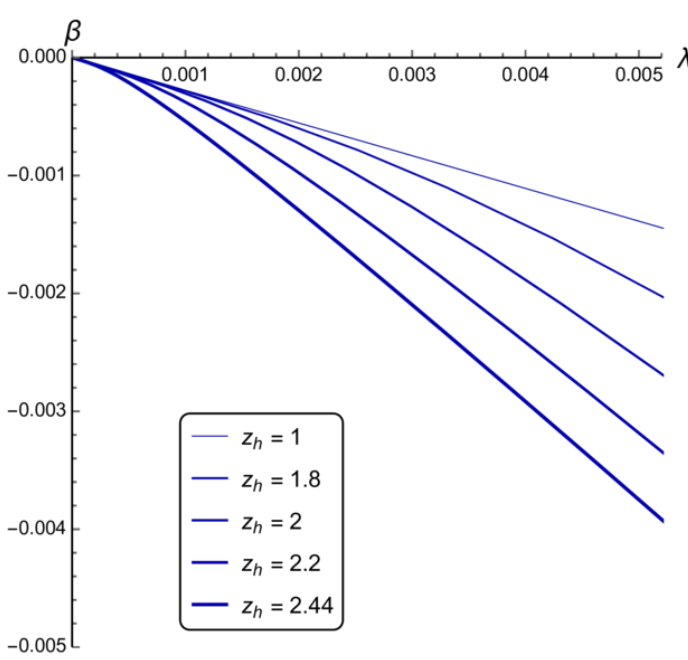

A

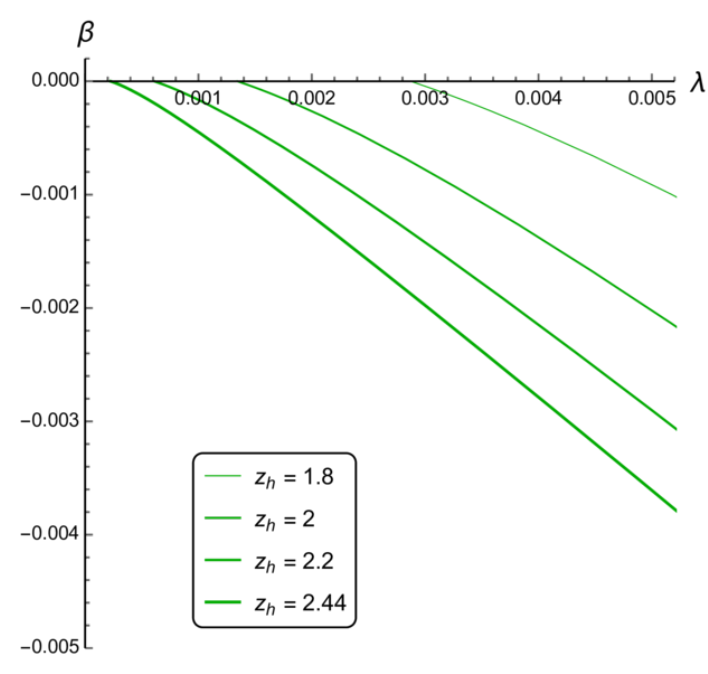

C

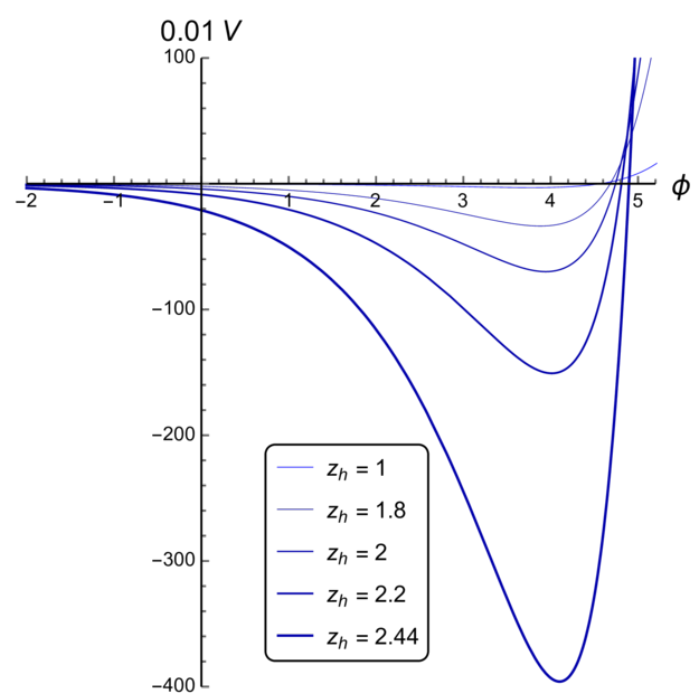

B

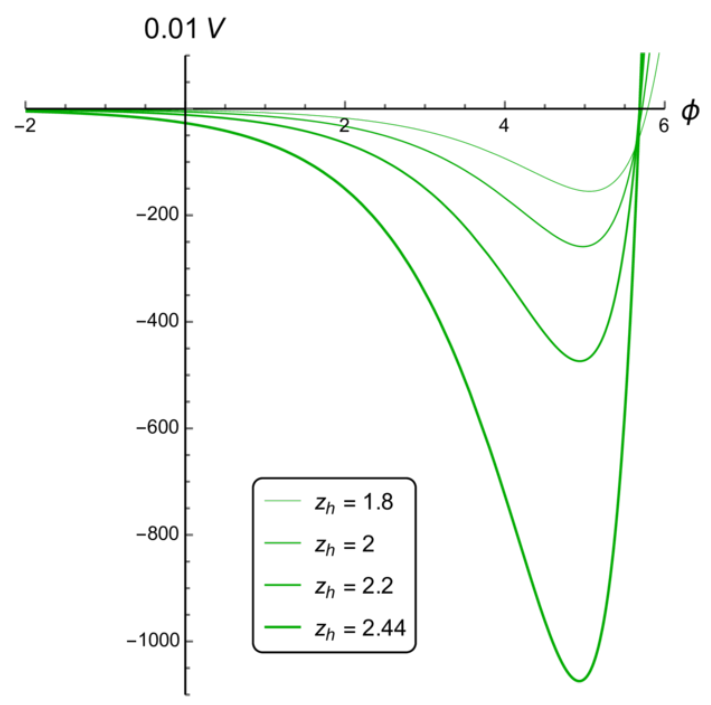

$\mathrm{D}$

Figure 13. The function $\beta(\lambda)$ in anisotropic, $\nu=4.5,(\mathrm{~A})$ and isotropic, $\nu=1,(\mathrm{C})$ cases near small $\lambda$; scalar potentials $V(\phi)$ for $\mu=0$ in anisotropic, $\nu=4.5,(\mathrm{~B})$ and isotropic, $\nu=1$, (D) cases.

In figure 15 the $Y$-flow is shown for anisotropic case with zero and non-zero chemical potential. The function grows rapidly and this growth does not essentially depend on the size of the horizon.

In figure 16 we display the RG flows in the $(X, Y)$-plane for anisotropic case $\nu=4.5$ and zero and non-zero chemical potential. The $X$ and $Y$ have the inverse ratio dependence and does not change much for different $z_{h}$.

Figure 17 shows the $\mathrm{RG}$ flows in the $(X, Y, H)$-space. We see that our anisotropy essentially changes the character of the flow. 


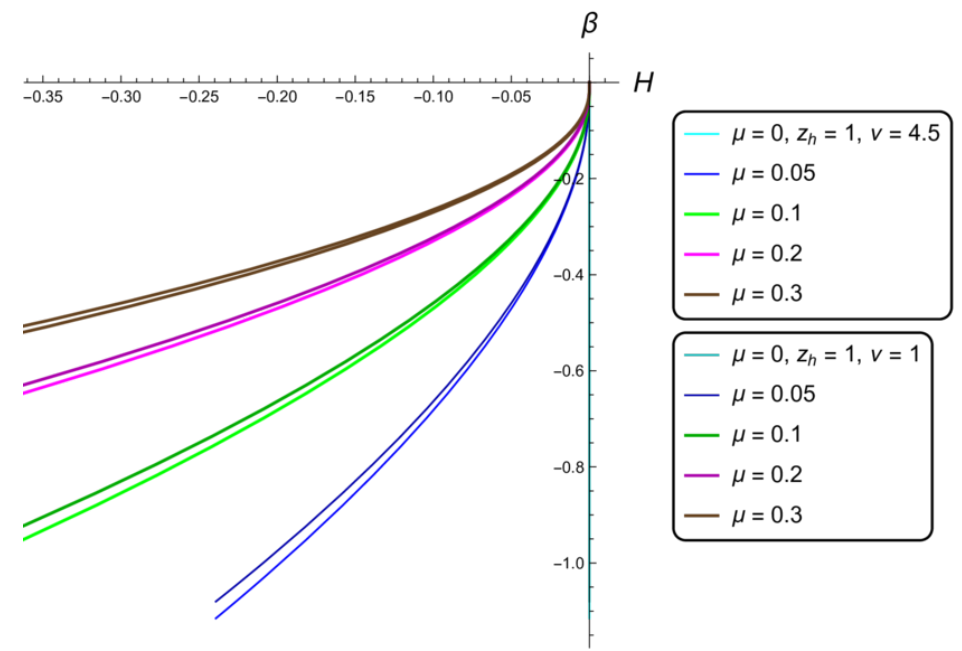

Figure 14. The function $\beta(H)$ in isotropic and anisotropic $\nu=4.5$ cases.

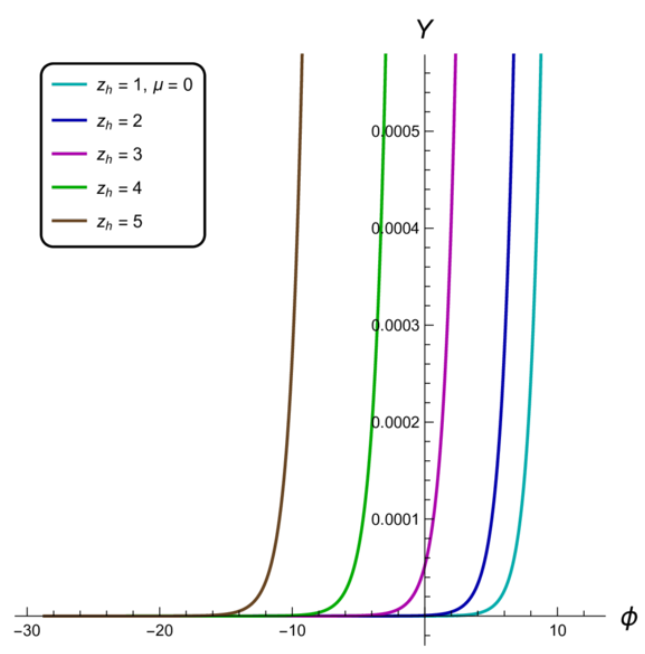

A

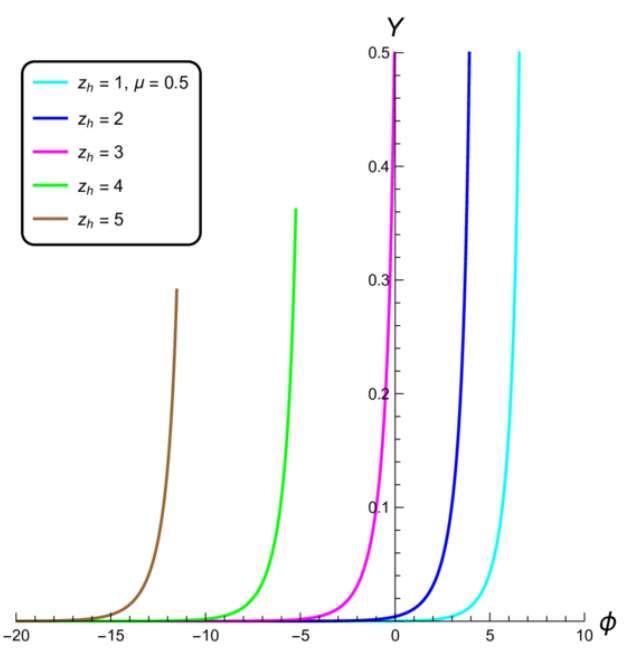

$\mathrm{B}$

Figure 15. $Y$ flows in anisotropic case $\nu=4.5$ for different $z_{h}, \mu=0$ (A) and $\mu=0.5$ (B).

\section{Thermodynamics of the background}

\subsection{Temperature}

Calculating the derivative of the blackening function (2.48) at the horizon we get the temperature

$$
T\left(z_{h}, \mu, c, \nu\right)=\frac{g^{\prime}\left(z_{h}\right)}{4 \pi}=\frac{e^{-\frac{3 c z_{h}^{2}}{4}}}{2 \pi z_{h}}\left|\frac{1}{\mathfrak{G}\left(\frac{3}{4} c z_{h}^{2}\right)}+\frac{\mu^{2} c z_{h}^{2+\frac{2}{\nu}} e^{\frac{c z_{h}^{2}}{4}}}{4\left(1-e^{\frac{c z_{h}^{2}}{4}}\right)^{2}}\left(1-e^{\frac{c z_{h}^{2}}{4}} \frac{\mathfrak{G}\left(c z_{h}^{2}\right)}{\mathfrak{G}\left(\frac{3}{4} c z_{h}^{2}\right)}\right)\right|
$$




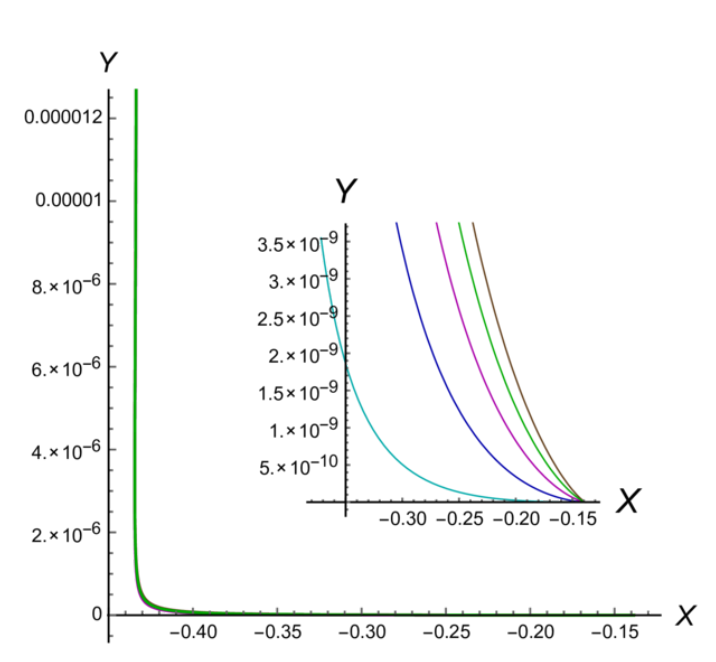

A

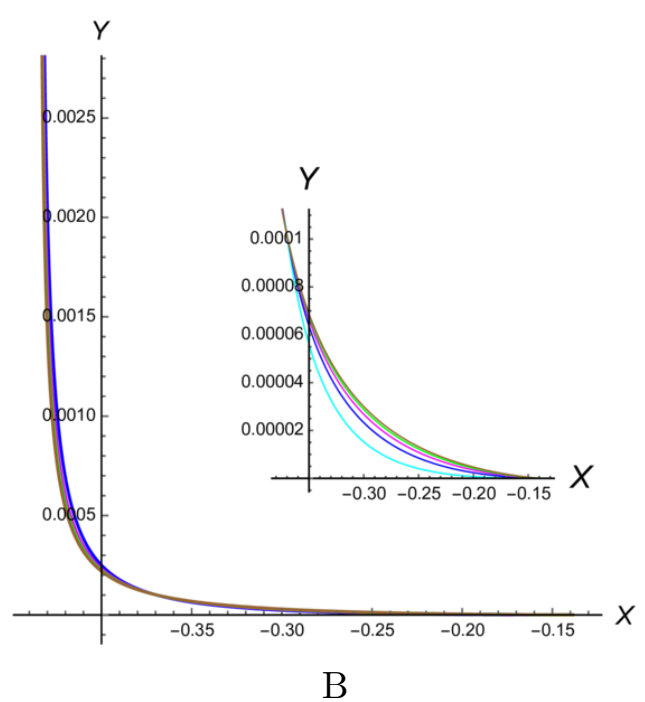

$\mathrm{B}$

Figure 16. RG flows in the (X,Y)-plane in anisotropic case $\nu=4.5$ for $\mu=0$ (A) and $\mu=0.5$ (B); the plot legends are the same as in figure 15.

Here the dependence on $\nu$ is caused by the function $\mathfrak{G}$ (2.39) in the right-hand side of (3.1). In particular, for the zero chemical potential, $\mu=0$,

$$
T\left(z_{h}, c, \nu\right) \equiv T\left(z_{h}, 0, c, \nu\right)=\frac{e^{-\frac{3 c z_{h}^{2}}{4}}}{2 \pi z_{h} \mathfrak{G}\left(\frac{3}{4} c z_{h}^{2}\right)} .
$$

For $c=0$ it reproduces the result from [33]:

$$
T\left(z_{h}, 0, \nu\right)=\lim _{c \rightarrow 0} \frac{e^{-\frac{3 c z_{h}^{2}}{4}}}{2 \pi z_{h} \mathfrak{G}\left(\frac{3}{4} c z_{h}^{2}\right)}=\frac{1}{2 \pi z_{h}} \frac{\nu+1}{\nu} .
$$

From (3.1) we get the dependence of temperature on $z_{h}, \mu, c$ and $\nu$. In figure 18.A and figure 18.B we present the dependence of $T$ on $z_{h}$ for different $\mu$ and fixed $c=-1$ for isotropic (figure 18.A) and anisotropic (figure 18.B) cases, respectively. In figure 18.C we compare the plots, presented in figure 18.A and figure 18.B. Plot in figure 18.D is a zoom of figure 18.B. In figure 19 we present the dependence of temperature on $z_{h}$ for different $\mu=0$ and $c<0$, and in figure 20 the dependence of temperature on $\mu$ keeping $z_{h}=1$ for different $c<0$ is shown. Figure 21 displays contour plots for the temperature dependence on the horizon position and chemical potential for the isotropic (A) and anisotropic (B) cases at fixed $c=-1$.

These plots show the following behavior of the temperature:

- For $\mu=0$ (dashed lines) there is one extremal point (minimum) for the temperature as a function of the horizon position; we denote the corresponding horizon $z_{h}$ as $z_{h, \min }(0)=z_{h, \min }(0, c, \nu)$, and we get the following picture:

- for $0<z_{h}<z_{h, \min }(0)$ (large black holes) the temperature drops as $z_{h}$ grows and for $z_{h, \min }(0)<z_{h}$ (small black holes) the temperature increases with the growth of $z_{h}$; 


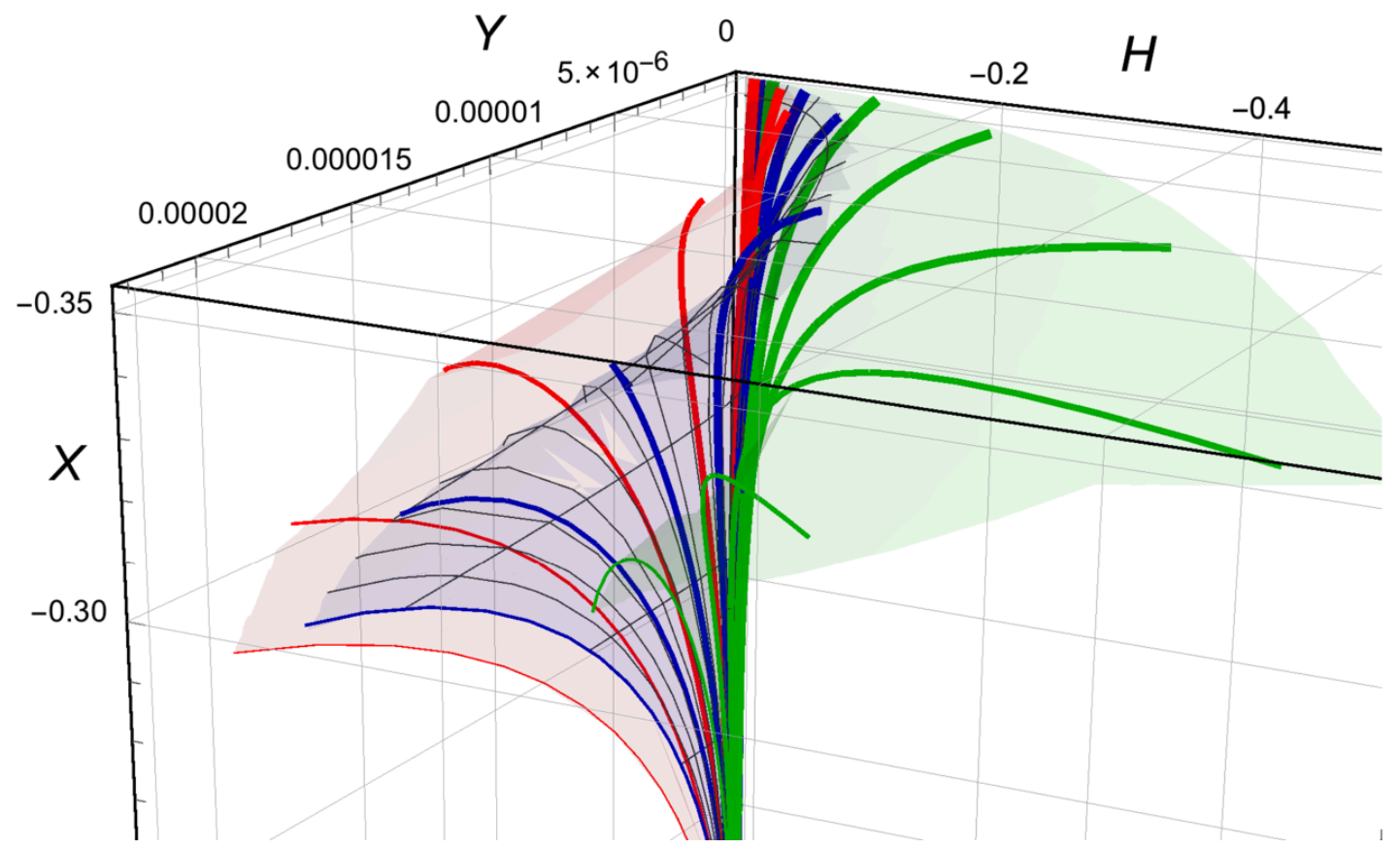

A

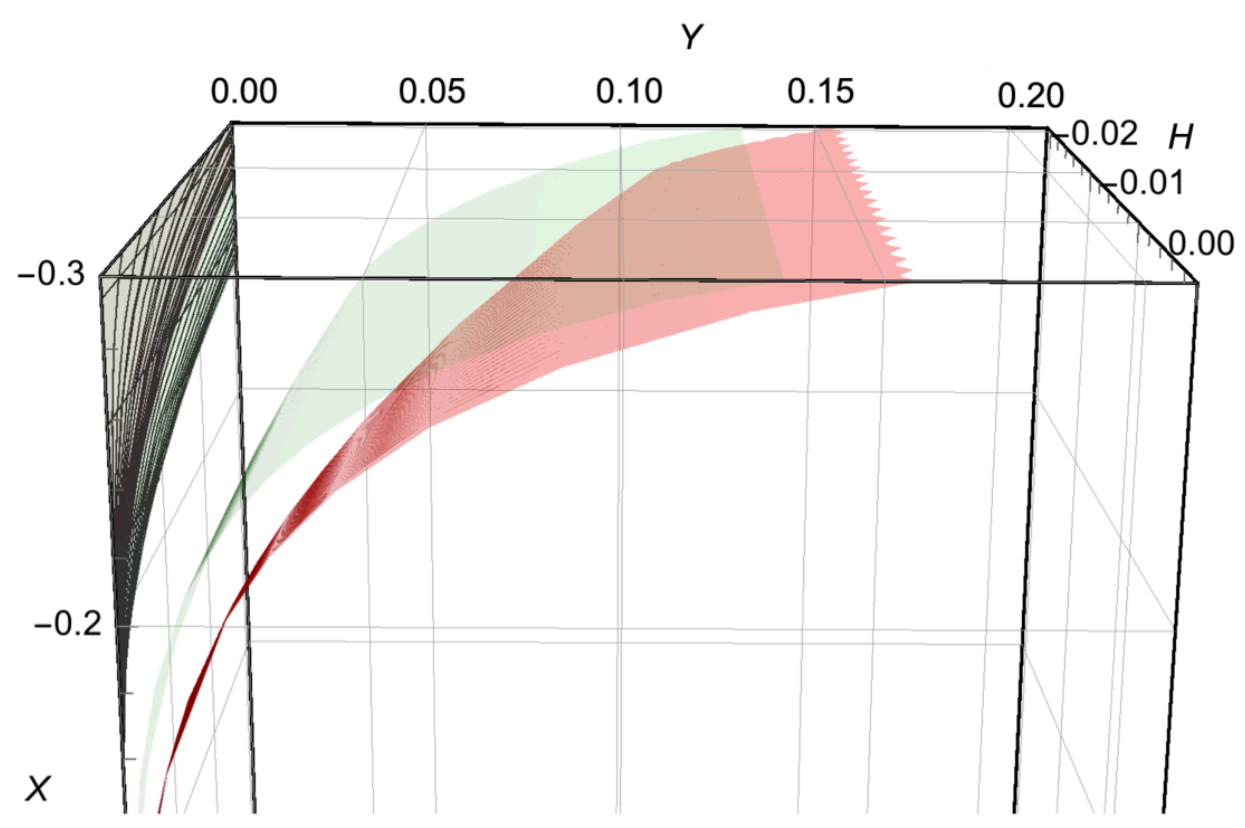

B

Figure 17. RG flows in the $(X, Y, H)$-space in anisotropic case $\nu=4.5$ for $z_{h}=2$ (light red), $z_{h}=1.5$ (light blue) and $z_{h}=1$ (lightgreen) (A); RG flows in the $(X, Y, H)$-space in anisotropic, $\nu=4.5$, (meshed lines) and isotropic cases (not meshed lines) for $z_{h}=1$ (lightgreen) and $z_{h}=2$ (lightred) (B). 
- the minimal isotropic horizon $z_{\min }^{\text {(iso) }}(c) \equiv z_{h, \min }(0, c, 1)$ is larger than the minimal anisotropic horizon $z_{\min }^{(\nu)}(c) \equiv z_{\min }(0, c, \nu)$, i.e. $z_{\min }^{(\text {iso })}(c)>z_{\min }^{(\nu)}(c)$ and the corresponding critical temperature $T_{\min }(0)$ is higher for the isotropic case, i.e. $T\left(z_{\min }^{\text {(iso) }}(c), c, 1\right) \equiv T_{\min }^{(\text {iso })}>T_{\min }^{(\nu)} \equiv T\left(z_{\min }^{(\nu)}(c), c, \nu\right)$; one can read these inequalities from the plot in figure 18.C and D;

- for negative $c$ with decreasing $|c|$ the temperature $T_{\min }^{(\text {iso) }}$ (the brown dashed line) is below the green one and its minimum is shifted to the right from the minimum of the green one (figure 19.A); $T_{\text {min }}^{(\nu)}$ (the cyan dashed line) is below the blue one and its minimum is shifted to the right from that of the blue one figure 18.B);

- for $c=-1$ the values of the minimal horizons $z_{\min }(\nu)$ are $z_{\min }^{\text {(iso) }}=1.547$ and $z_{\min }^{(4.5)}=1.168$ for the isotropic and anisotropic cases; the corresponding temperatures are $T_{\min }^{(\text {iso })}=0.355$ and $T_{\min }^{(4.5)}=0.245$ (figure 19.A and B).

- For $0<\mu<\mu_{\mathrm{cr}}$ there are two extremal points $z_{h, \min }(\mu)=z_{h, \min }(\mu, c, \nu)$ and $z_{h, \max }=$ $z_{h, \max }(\mu, c, \nu)$; corresponding $T_{\min }(\mu)$ and $T_{\max }(\mu)$ are shown in figure 18:

- for $0<z<z_{h, \text { min }}$ the temperature drops with the growth of $z_{h}$;

- for $z_{h, \min }<z<z_{h, \max }$ the temperature increases with the growth of $z_{h}$;

- for $z_{h, \text { max }}<z<z_{h_{0}}$ the temperature decreases again with the growth of $z_{h}$; here $z_{h_{0}}$ is the position of the new horizon, $T\left(z_{h_{0}}\right)=0, z_{h_{0}}=z_{h_{0}}(\mu, c, \nu)$;

$-\mu_{\mathrm{cr}}, z_{h, \min }$ and $z_{h, \max }$ depend on the warp factor coefficient $c$ and the anisotropic parameter $\nu$;

- the anisotropy increases the size of the new horizon, $z_{h_{0}}(\mu, c, 1)<z_{h_{0}}(\mu, c, \nu)$.

- For $\mu=\mu_{\mathrm{cr}}$ (dotted lines in figure 18) there is no extremal point, but there is an inflection point $\left(z_{h, \mathrm{cr}}, T_{\mathrm{cr}}\right), z_{h, \mathrm{cr}}=z_{h, \mathrm{cr}}\left(\mu_{\mathrm{cr}}\right), T_{\mathrm{cr}}=T_{\mathrm{cr}}\left(\mu_{\mathrm{cr}}\right)$, therefore

- for all values of $z_{h}$ with its growth the temperature decreases;

- the temperature becomes equal to zero at a new horizon.

- For $\mu>\mu_{\mathrm{cr}}$ increasing $z_{h}$ we decrease the temperature and there is a point $z_{h_{0}}=$ $z_{h_{0}}(\mu, c, \nu)$ where $T\left(z_{h_{0}}\right)=0$, i.e. a new horizon appears;

- for negative $c$ with the growth of $|c|$ the values of $z_{h_{0}}(\mu, \nu)(c)$ decrease, $z_{h_{0}}(\mu, \nu)\left(c_{1}\right)<z_{h_{0}}(\mu, \nu)\left(c_{2}\right)$ for $\left|c_{1}\right|>\left|c_{2}\right|$ and all $\nu \geq 1$ (figure 19).

We can also investigate the behavior of $T(\mu)$ using expression (3.1) and taking some fixed values of the horizon. In figure 20 we plot the curves for different negative values of the warp factor coefficient $c$ in isotropic (A) and anisotropic (B) cases for $z_{h}=1$. In figure 20.C we compare these cases plotting them together. Figure 20.D and E display $T(\mu)$ for large black holes with $z_{h}=0.5$ and small black holes with $z_{h}=1.5$. The function $T(\mu)$ decreases faster for smaller $c$. The isotropic case curves lie higher than the anisotropic ones and reach zero temperature at larger chemical potential values (figure 20.C). For smaller horizons we have the same picture (figure 20.D and E). 


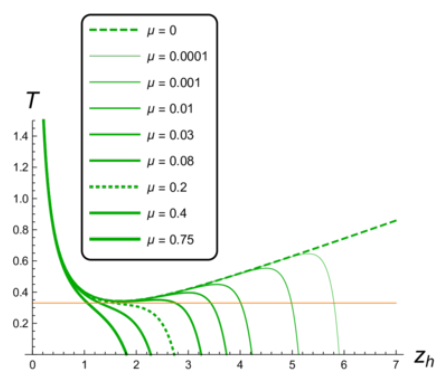

$\mathrm{A}$

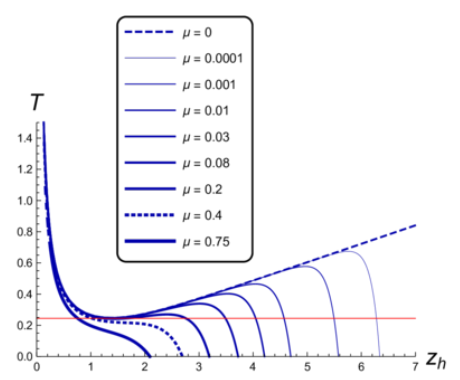

$\mathrm{B}$

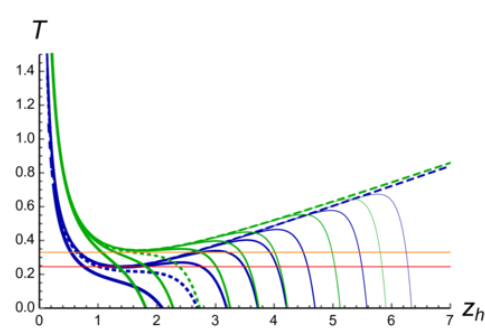

C

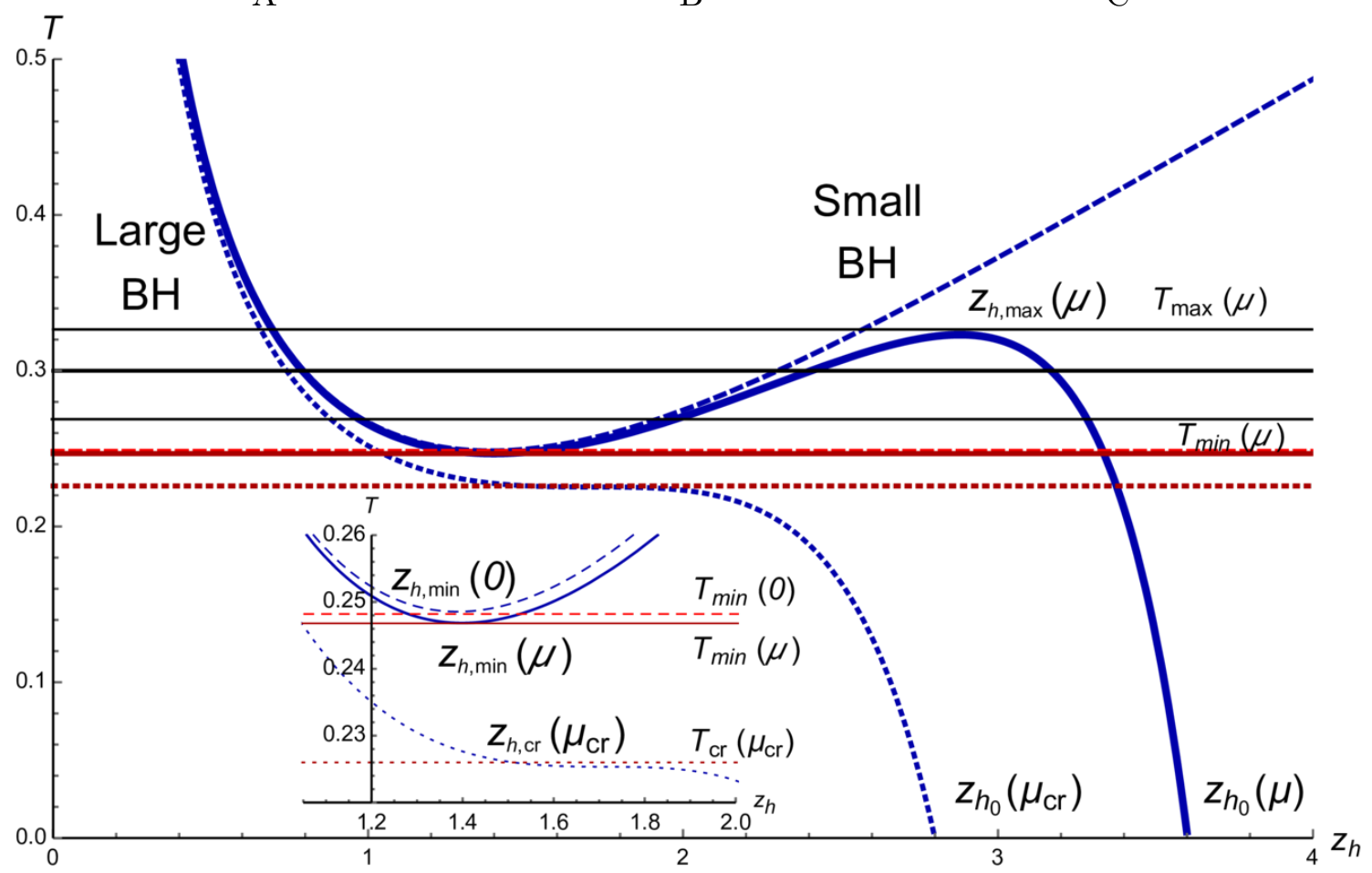

$\mathrm{D}$

Figure 18. The dependence of temperature $T\left(z_{h}, \mu, c, \nu\right)$ on $z_{h}$ for $c=-1$ and different $\mu$ for isotropic (A) and anisotropic, $\nu=4.5$, (B) cases, their comparison with the same line labels (C); anisotropic case for $c=-1$ and different $\mu$ in details (D); the horizontal orange and red lines show locations of the global minima in isotropic and anisotropic cases, respectively.

To summarize, note that the Van der Waals type of the temperature-horizon dependence $T\left(z_{h}\right)$ observed in $[45,46]$ for isotropic case, also takes place in the anisotropic one (see figure 21). In both cases this behavior becomes more pronounced with decreasing of negative $c$, see figure 19. The approximate solution considered in [35] does not inherit this property.

\section{$3.2 \quad$ Entropy}

The entropy is given by formula

$$
s\left(z_{h}, c, \nu\right)=\frac{e^{\frac{3}{4} c z_{h}^{2}}}{4} z_{h}^{-\frac{(\nu+2)}{\nu}}
$$

and is plotted in figure 22 . 


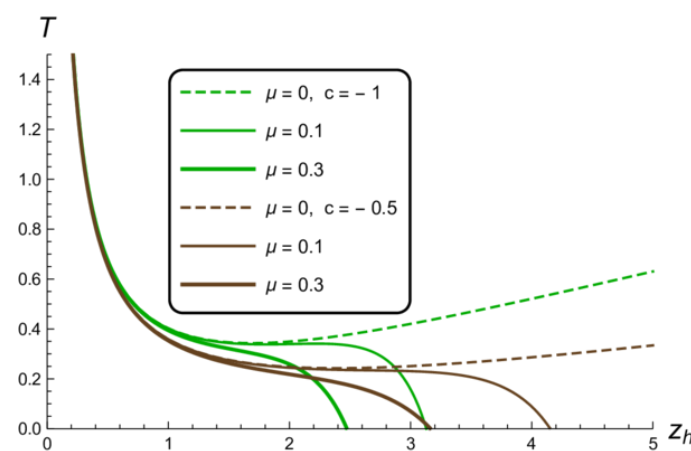

A

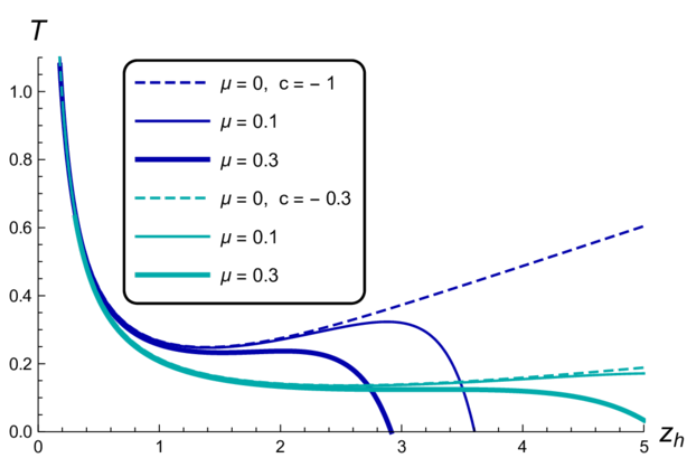

$\mathrm{B}$

Figure 19. File: Termo-mu-c-zh-original.nb+. The dependence of temperature $T\left(z_{h}, \mu, c, \nu\right)$ on $z_{h}$ for different $c$ and $\mu$ in isotropic (A) and anisotropic, $\nu=4.5,(\mathrm{~B})$ cases.

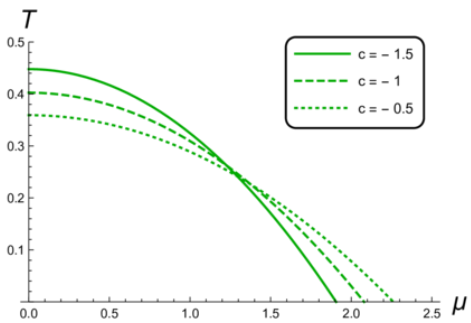

A

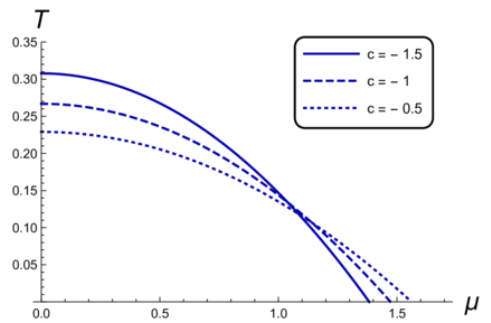

$\mathrm{B}$

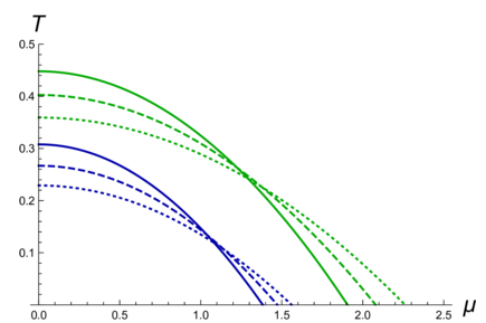

$\mathrm{C}$

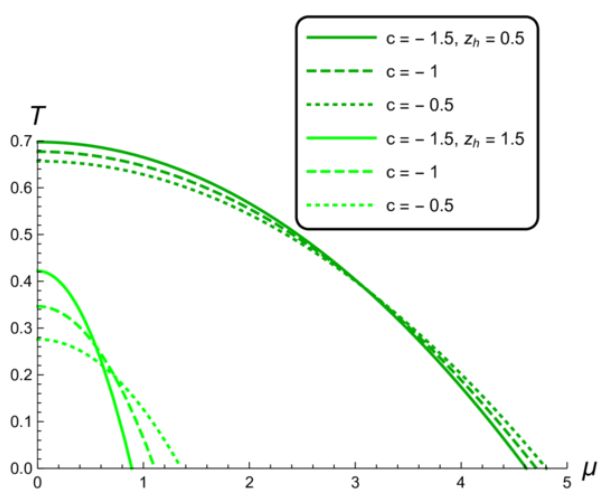

$\mathrm{D}$

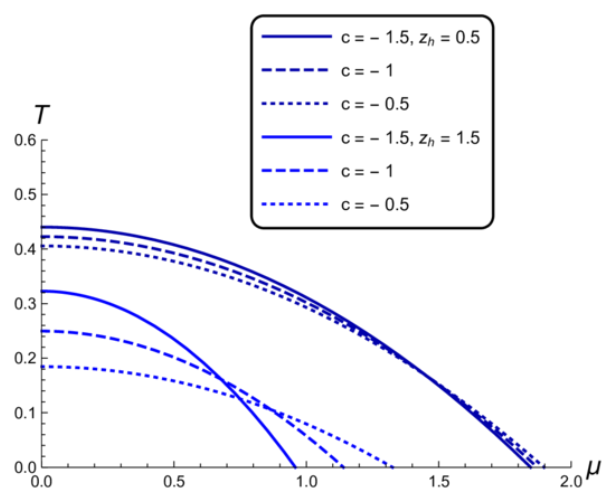

$\mathrm{E}$

Figure 20. The dependence of temperature $T\left(z_{h}, \mu, c, \nu\right)$ on $\mu$ for $z_{h}=1$ and different $c$ in isotropic (A) and anisotropic, $\nu=4.5$, (B) cases, their the comparison with the same line labels (C); for small black holes with $z_{h}=1.5$ and large black holes with $z_{h}=0.5$ and different $c$ in the isotropic (D) and anisotropic, $\nu=4.5,(\mathrm{E})$ cases.

Figure 22.A shows that the entropy is a monotonously decreasing function of the horizon $z_{h}$ both for the isotropic and the anisotropic cases, in other words the entropy values are bigger for larger black holes, whose horizons are smaller. As we see from figure 22.B, the velocity of the entropy decreasing depends on parameters $c$ and $\nu$. It is interesting to note that absolute value of this velocity for the same $c$ is bigger in the isotropic case for 


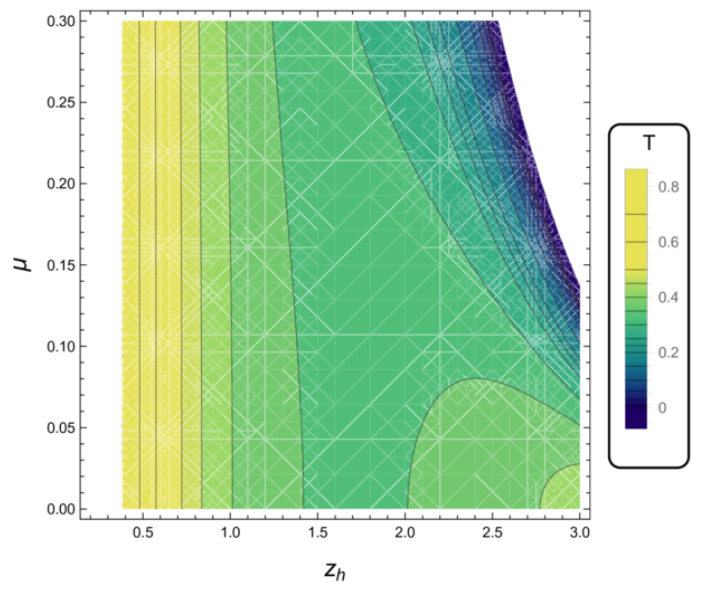

A

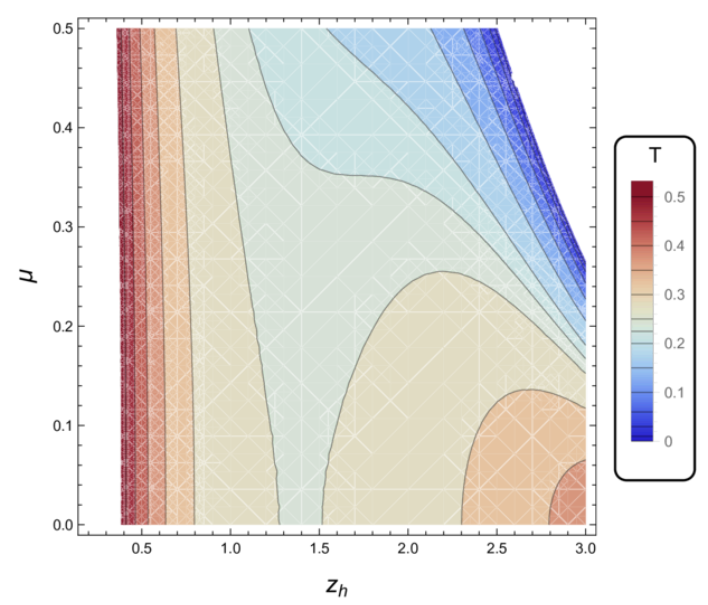

$\mathrm{B}$

Figure 21. Contour lines of the temperature $T=T\left(z_{h}, \mu, c, \nu\right)$ for $c=-1$ in the isotropic (A) and anisotropic $\nu=4.5$ (B) cases.

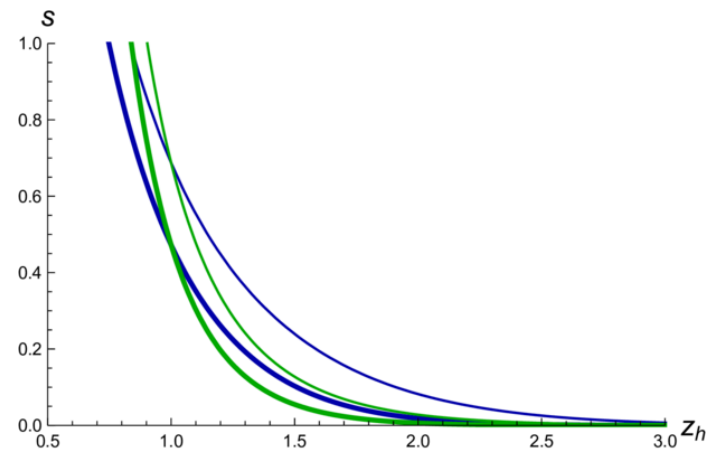

A

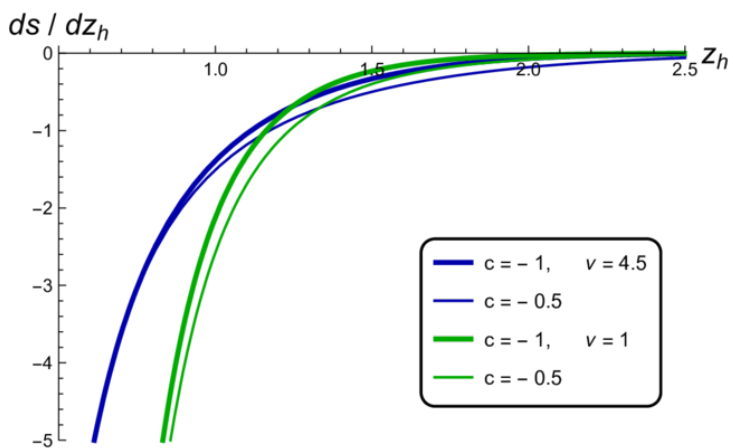

B

Figure 22. The black hole entropy $s\left(z_{h}\right)$ for $\mu=0$ and different $c$ in isotropic (green lines) and anisotropic (blue lines) (A) cases; the velocity of the entropy decreasing $\partial s\left(z_{h}\right) / \partial z_{h}$ (B); the plot legends are the same for both panels.

large black holes and is smaller for small black holes. More precisely, for $c=-1$

$$
\begin{array}{ll}
s_{z_{h}}^{\prime}\left(z_{h},-1,1\right)<s_{z_{h}}^{\prime}\left(z_{h},-1,4.5\right)<0 & \text { for } \quad z_{h}<1.248 \\
s_{z_{h}}^{\prime}\left(z_{h},-1,4.5\right)<s_{z_{h}}^{\prime}\left(z_{h},-1,1\right)<0 & \text { for } \quad z_{h}>1.248
\end{array}
$$

and for $c=-0.5$

$$
\begin{array}{lll}
s_{z_{h}}^{\prime}\left(z_{h},-0.5,1\right)<s_{z_{h}}^{\prime}\left(z_{h},-0.5,4.5\right)<0 & \text { for } \quad z_{h}<1.331 \\
s_{z_{h}}^{\prime}\left(z_{h},-0.5,4.5\right)<s_{z_{h}}^{\prime}\left(z_{h},-0.5,1\right)<0 & \text { for } \quad z_{h}>1.331 .
\end{array}
$$

Here $s_{z_{h}}^{\prime}\left(z_{h}, c, \nu\right)=\partial s\left(z_{h}, c, \nu\right) / \partial z_{h}$.

In figure 23 we present the entropy dependence on temperature $T$ for $\mu=0$ and different $c$ for isotropic (green lines) and anisotropic (blue lines) cases. The plots in figure 23 show that in both cases there are minimal temperatures for which the black holes exist. 


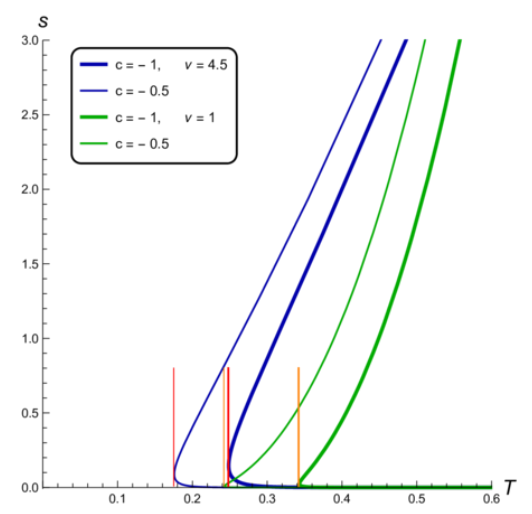

A
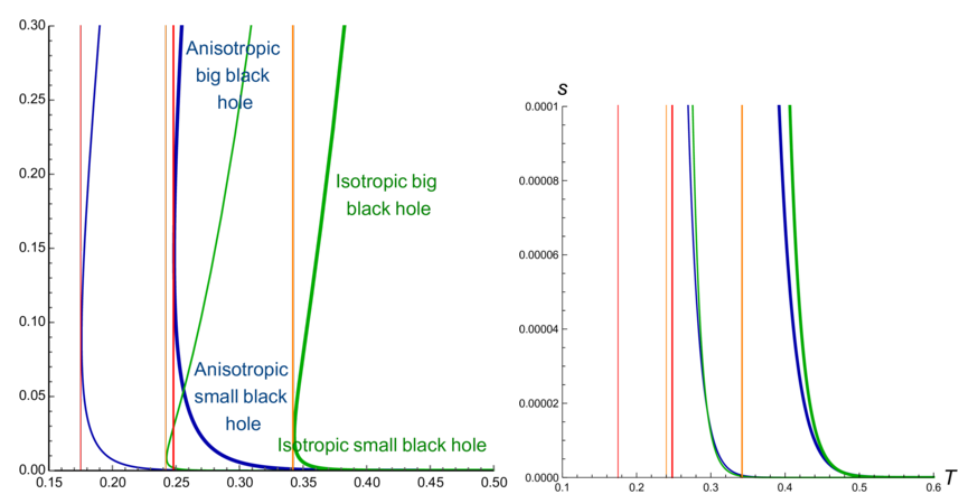

$\mathrm{B}$

Figure 23. The black hole entropy $s(T)$ for $\mu=0$ and different $c$ in isotropic (green lines) and anisotropic (blue lines) cases $(\mathrm{A})$; regions located near the minimum temperatures $T_{\min }(c, \nu)$ with an increased scale on the right $(\mathrm{B})$; the red vertical lines indicate $T_{\min }(c, 4.5)$ and the orange ones indicate $T_{\min }(c, 1)$ for $c=-0.5$ and $c=-1$.

The minimal temperature in the isotropic case is higher then in the anisotropic one for the same value of $c<0, T_{\min }(c, 4.5)<T_{\min }(c, 1)$, that agrees with the plots in figure 18. In both cases the entropy is a double-valued function and has a large black holes branches and a small black holes one. For the small black holes the entropy increases with decreasing $T$ thus leading to the negativity of the specific heat $c_{v}=T d s / d T$. Therefore small black holes are thermodynamically unstable, whereas entropy of the large black holes grows while temperature increases and therefore large black holes are thermodynamically stable.

In figure 24 we present the entropy dependence on temperature $T$ for $c=-1$ and different $\mu \geq 0$ for isotropic (green lines) and anisotropic (blue lines) cases. The plots in figure 24 show that in both cases for fixed $\mu, 0 \leq \mu \leq \mu_{\mathrm{cr}}(c, \nu)$, there are minimal $T_{\min }(\mu, \nu)$ and maximal $T_{\max }(\mu, \nu)$ temperatures, between which the entropy is a multivalued function of $T$ with three branches. We see well only two braches in figure 24.A, to see the third one has to draw the picture figure 24.B for small values of $s(T)$. The schematic picture of three branches is presented in figure 24.C. When we decrease the temperature, the entropy decreases along the first branch $\left(T_{\min }(\mu, \nu)<T<\infty\right)$. Then the entropy decreases along the second branch with an increase of temperature from $T_{\min }(\mu, \nu)$ to $T_{\max }(\mu, \nu)$, i.e. here the black holes are unstable. Finally the entropy increases along the third branch with an increase of temperature for $0 \leq T<T_{\max }(\mu, \nu)$, see also figure 18.D. In plots figure 25.A and figure 25 .B we show the unstable second branches as well as the transition of the threebranch solution to the unified one-branch solutions at $\mu=\mu_{\mathrm{cr}}(-1, \nu)$ (dotted green and blue lines for the isotropic and anisotropic cases). The entropy dependence on the temperature at unified branches corresponding to $\mu \geq \mu_{\mathrm{cr}}(c, \nu)$ is presented in plots figure 25 by lines for $\mu \geq 0.119$ in isotropic case and $\mu \geq 0.3$ in anisotropic case. 


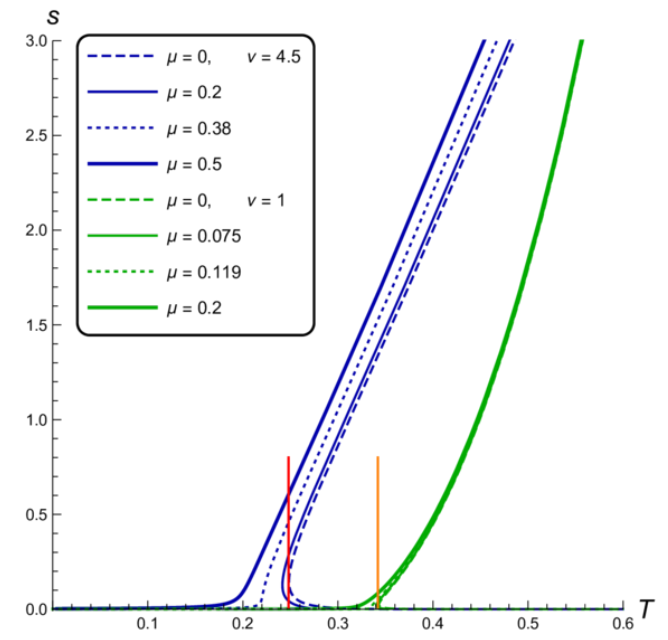

A

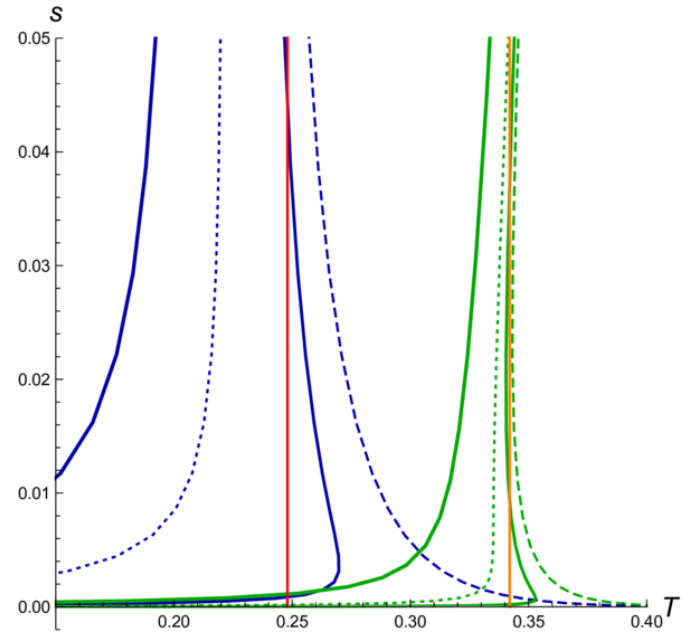

$\mathrm{B}$

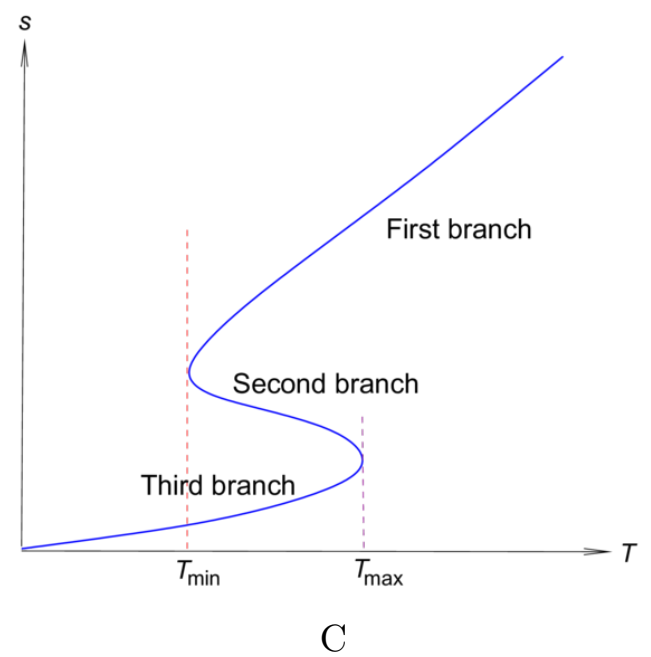

Figure 24. The black hole entropy $s(T)$ for different $\mu$ and $c=-1$ in isotropic (green lines) and anisotropic case (blue lines) (A); a region of (A), located near the minimum temperatures $T_{\min }(c, \nu)$, in increased scale (B), where the red vertical line indicates $T_{\min }(-1,4.5)=0.245$ and the orange one indicates $T_{\min }(-1,1)=0.355$ for $\mu=0$; the schematic view of $s(T)$ three-branches behavior $(\mathrm{C})$.

\subsection{Free energy}

To study transitions between different branches in more detail it is reasonable to consider the free energy behavior of the corresponding solutions. The free energy for a given chemical potential and fixed volume is related to the entropy as

$$
d F=-s d T
$$

and can be found by integration of (3.9) that gives

$$
F\left(z_{h}, c, \nu\right)=\int s d T=\int_{z_{h}}^{\infty} s\left(z_{h}, c, \nu\right) T^{\prime}\left(z_{h}, c, \nu\right) d z_{h} .
$$

The dependence of the free energy on the horizon position $z_{h}$ is presented in figure 26 , the dependence on $T$ is presented in figure 27 and figure 28. In figure 26. A, which corresponds 


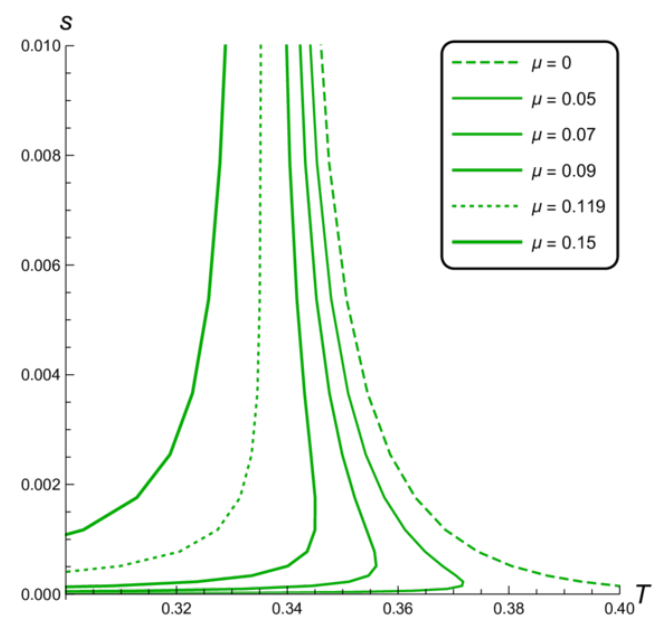

A

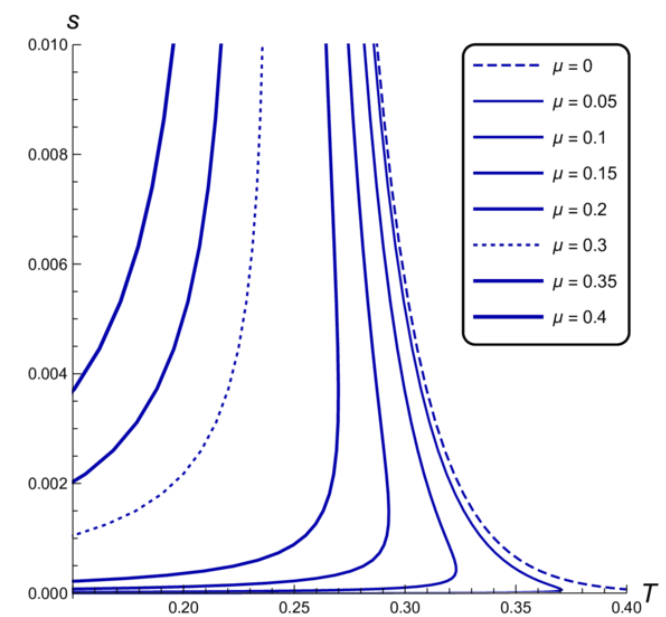

$\mathrm{B}$

Figure 25. Unstable second branches of $s(T)$ in isotropic (A) and anisotropic case (B) cases for $c=-1$ and different $\mu$.

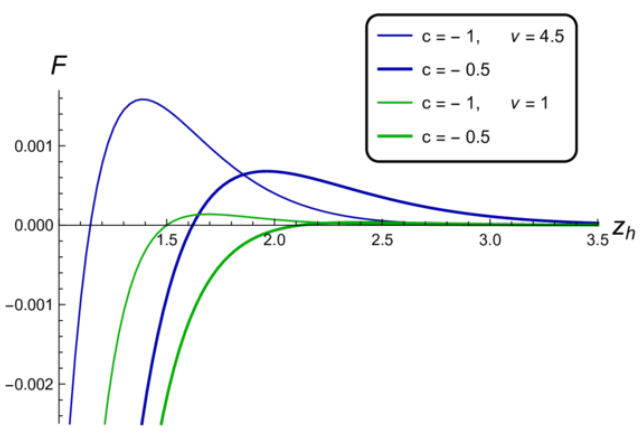

A

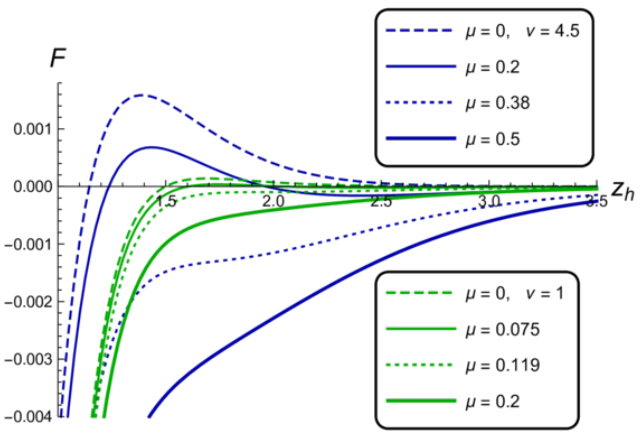

$\mathrm{B}$

Figure 26. The black hole free energy $F\left(z_{h}\right)$ in isotropic (green lines) and anisotropic (blue lines) cases for different $c, \mu=0(\mathrm{~A})$ and different $\mu \geq 0, c=-1(\mathrm{~B})$. The intersections with the horizontal axis give the values of the Hawking-Page horizons $z_{h, H P}(\mu, c, \nu)$.

to $\mu=0$, we can see that the free energy as the function of $z_{h}$ is equal to zero at $z_{h}=$ $z_{h, H P}(0, c, \nu)$, and at this point the Hawking-Page phase transition takes place. The value $z_{h, H P}(0, c, \nu)$ depends on $c$ and $\nu$, and $z_{h, H P}\left(0, c_{1}, \nu\right)<z_{h, H P}\left(0, c_{2}, \nu\right)$ for $c_{1}<c_{2}<0$. For the anisotropic background the Hawking-Page horizon is less than for the isotropic one with the same $c<0$. In particular,

$$
1.138=z_{h, H P}(0,-1,4.5)<z_{h, H P}(0,-1,1)=1.505 .
$$

Note that the position of horizon, where the temperature gets its local minimum, exceed the position of the Hawking-Page horizon $z_{h, H P}(0,-1, \nu)<z_{h, \min }(0,-1, \nu)$.

As we can see from the plots in figure 26.B, for $0<\mu<\mu_{\mathrm{cr}, H P}(c, \nu)$ the free energy as the function of the horizon position keeps the same behavior as for $\mu=0$. At $\mu=$ $\mu_{\mathrm{cr}, H P}(c, \nu)$ the free energy becomes non-positive and for $\mu>\mu_{\mathrm{cr}, H P}(c, \nu)$ the Hawking- 


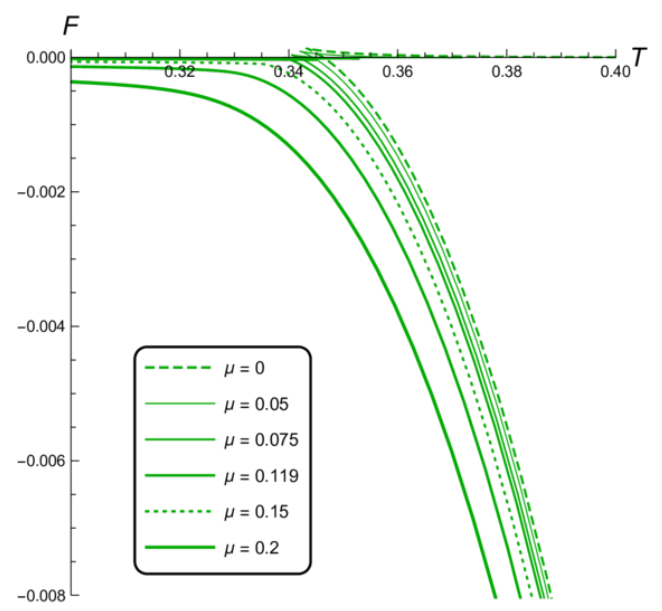

A

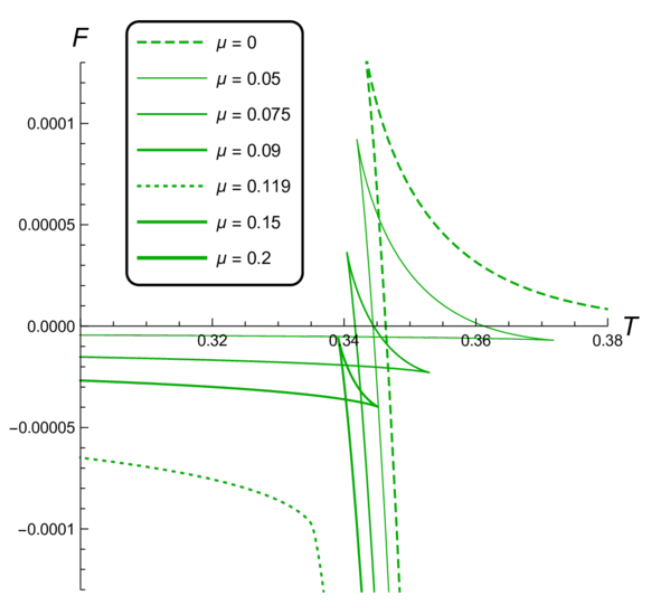

$\mathrm{B}$

Figure 27. The black hole free energy $F(T)$ for $c=-1$ and different $\mu$ in isotropic case (A) and its zoom near $F=0(\mathrm{~B})$.

Page horizon disappears. But for the chemical potential values in the interval $\mu_{\mathrm{cr}, H P}(c, \nu)<$ $\mu<\mu_{\mathrm{cr}}(c, \nu)$, the free energy still is double-valued what causes the black hole to black hole phase transition (see below).

In figure 27 and figure 28 we show the behavior of the free energy as function of the temperature. At $\mu=0$, as we can see in figure 27.B and figure 28.D, the free energy plots intersect the horizontal axis at $T_{H P}(c, \nu)$, where the Hawking-Page phase transitions take place, $T_{H P}(0,-1,1)=0.347$ and $T_{H P}(0,-1,4.5)=0.256$. At $T_{H P}$ black holes dissolve to thermal gas states with $F_{\text {gas }}=0$. We note that $T_{H P}(0,-1,4.5)<T_{H P}(0,-1,1)$, and comparing to $T_{\min }(0,-1,4.5)=0.255$ and $T_{\min }(0,-1,1)=0.345$ we conclude that in both isotropic and anisotropic cases,

$$
\begin{gathered}
0.347=T_{H P}(0,-1,1)>T_{\min }(0,-1,1)=0.345 \\
0.256=T_{H P}(0,-1,4.5)>T_{\min }(0,-1,4.5)=0.255 .
\end{gathered}
$$

From figure 26 we see that for zero chemical potential the free energy increases with $z_{h}$ growth for large black holes, i.e. for $z_{h}<z_{h_{\mathrm{cr}}}(c, \nu)$, and decreases for small black holes.

For $0<\mu<\mu_{\mathrm{cr}}(c, \nu)$ the dependence of the free energy from the temperature looks like the swallow-tailed shape both in isotropic and anisotropic cases. When we decrease the temperature from very large values up to $T_{\min }(\mu)\left(T_{\min }(\mu)=T_{\min }(\mu, c, \nu)\right.$, see figure 18), the free energy riches its maximum value, then goes down to its local minimum at $T_{\max }(c, \nu)$ and turns back to increase. It intersects itself at $T=T_{B B}(\mu)$, where a large black hole transits to a small one. Since both free energy values at $T=T_{B B}$ are equal and negative, meanwhile the free energy of the thermal gas is zero, the system undergoes the phase transition not to a thermal gaz, but to small black hole background. When we increase the chemical potential $\mu$ from zero to $\mu_{\mathrm{cr}}$, the loop of the swallow-tailed shape shrinks 


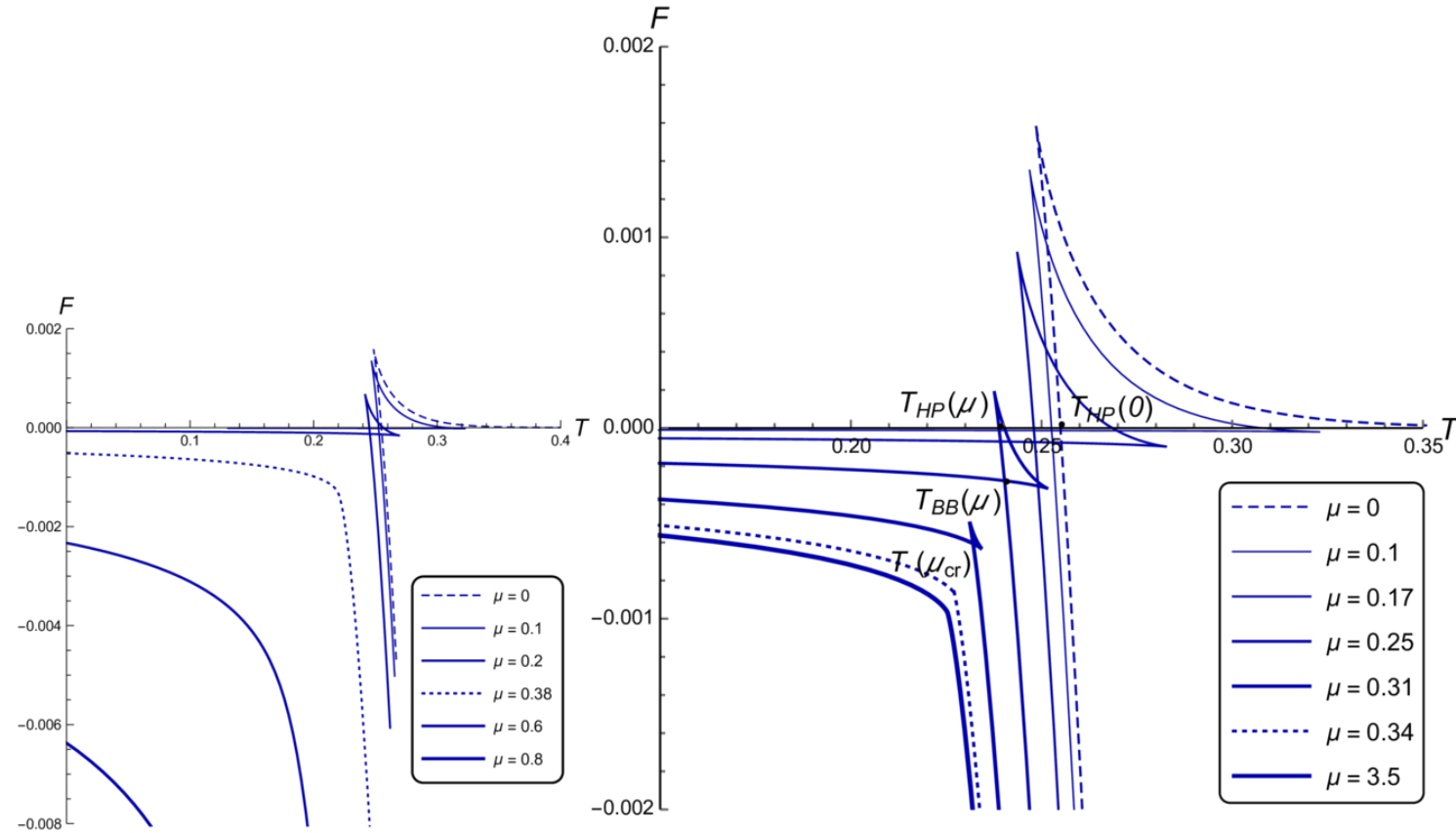

A

$\mathrm{B}$

Figure 28. The black hole free energy $F(T)$ for $c=-1$ and different $\mu$ in anisotropic case, $\nu=4.5$, (A) and its zoom near $F=0$ (B).

to disappear at $\mu=\mu_{\mathrm{cr}}(c, \nu)$. For $\mu>\mu_{\mathrm{cr}}(c, \nu)$, the curve of the free energy increases smoothly from higher to lower values of temperature.

It is interesting to compare the phase diagrams corresponding to isotropic and anisotropic backgrounds, see figure 29. We see that the first order phase transitions start at $\left(0, T_{H P}(0,1)\right)$ and $\left(0, T_{H P}(0,4.5)\right)$, so that $T_{H P}(0,4.5)<T_{H P}(0,1)$ and the transition lines describing transitions from large black holes to small ones stop at points $\left(\mu_{\mathrm{cr}}^{(\text {iso })}, T_{\mathrm{cr}}^{\text {(iso) }}\right.$ ) and $\left(\mu_{\mathrm{cr}}^{(\text {aniso })}, T_{\mathrm{cr}}^{(\text {aniso })}\right)$, herewith $\mu_{\mathrm{cr}}^{(\text {(iso })}<\mu_{\mathrm{cr}}^{(\text {aniso })}$ and $T_{\mathrm{cr}}^{(\text {iso })}>T_{\mathrm{cr}}^{(\text {aniso })}$.

It is also important for us to know the position of the large black holes to small black holes transition points at $\left(z_{h}, T\right)$-plane, see figure 30 . In these plots the horizontal arrows show transitions from the large black holes to small black holes for the anisotropic $\nu=4.5$ and isotropic cases. The shaded by these arrow areas define the instability zones.

Figure 31 summarizes our discussion of the phase transitions of our black hole isotropic and anisotropic backgrounds. In the next section we put probe strings in these backgrounds to find out information about the confinement/deconfinement phase transition.

\section{Confinement-deconfinement phase transition}

\subsection{Equation for the dynamical wall}

To guarantee the confinement-deconfinement phase transition one has to check the existence of the dynamical wall (DW). The dynamical wall position $z_{D W}$ is defined by the minimal 


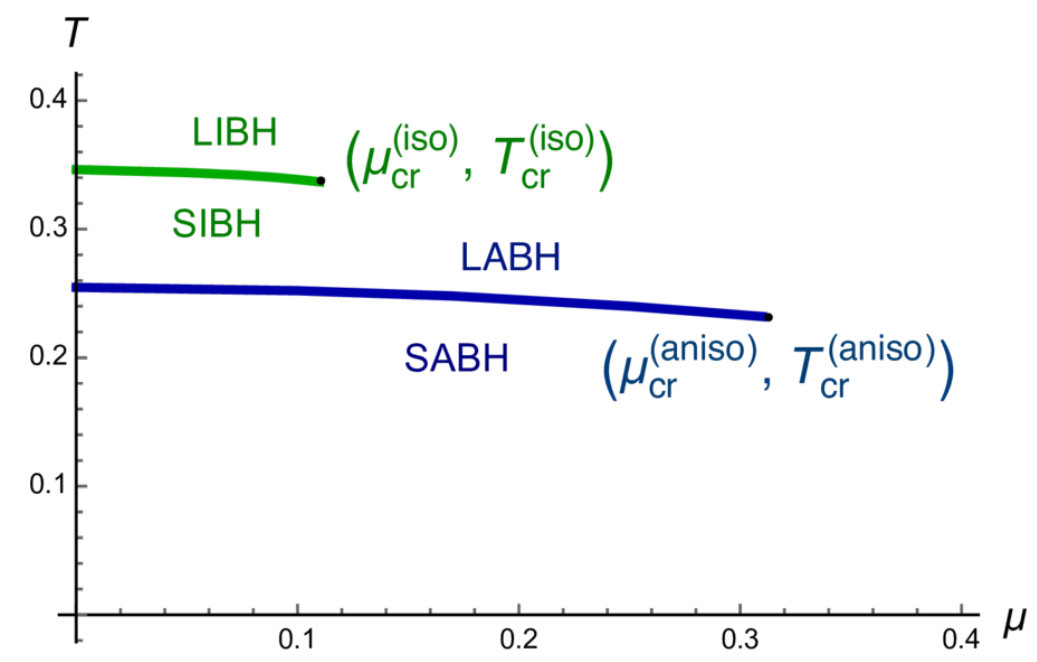

Figure 29. The phase diagrams in the $(T, \mu)$-plane for the isotropic background (green line) and for anisotropic $\nu=4.5$ background (blue line). LIBH and SIBH (LABH and SABH) indicate the regions of small and large isotropic (anisotropic) black holes.

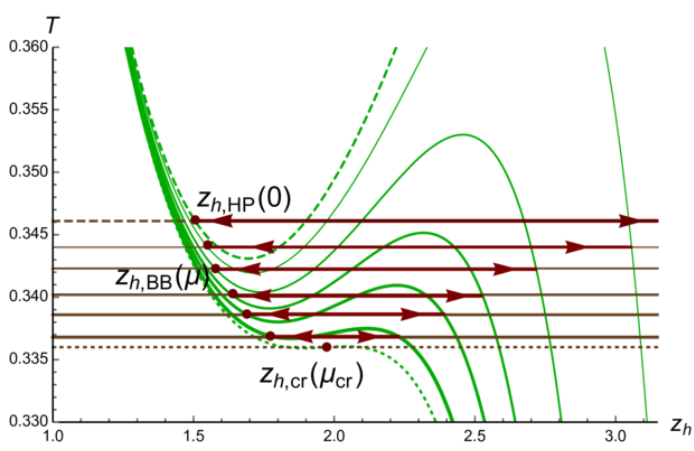

A

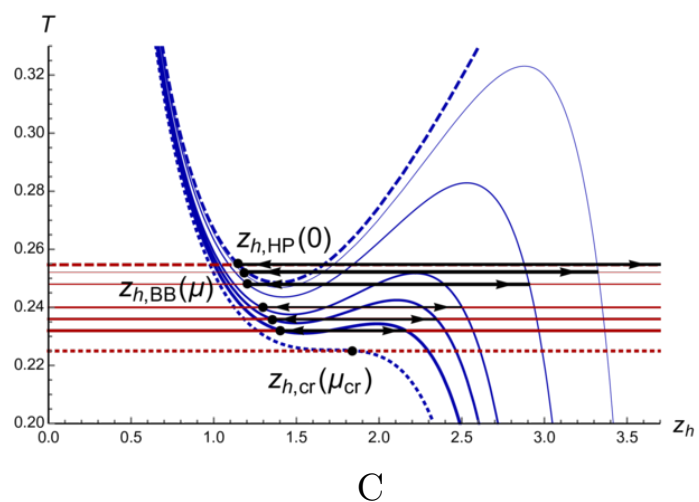

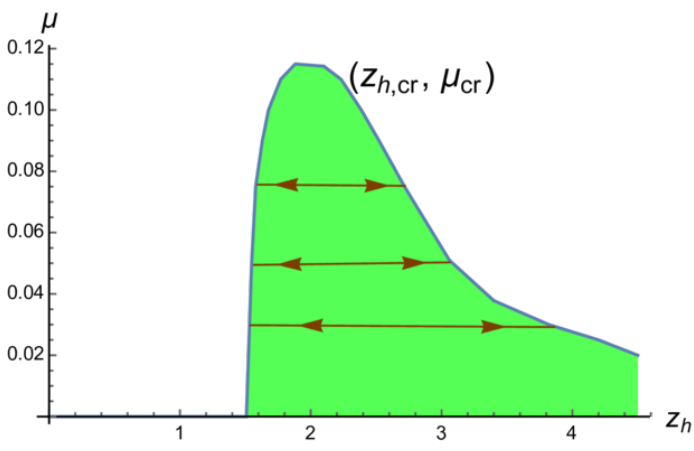

$\mathrm{B}$

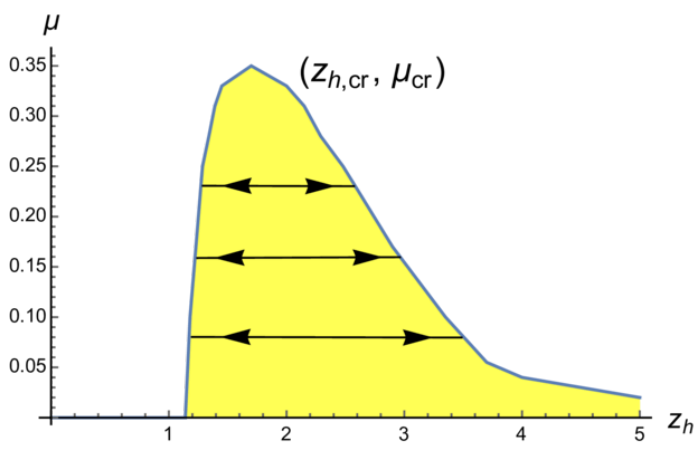

$\mathrm{D}$

Figure 30. The arrows show transitions large/small black holes for the isotropic (A) and anisotropic $\nu=4.5(\mathrm{C})$ cases in the $\left(z_{h}, T\right)$-plane; the large/small holes transitions in the $\left(z_{h}, \mu\right)$-plane are shown by arrows for isotropic (B) and anisotropic (D) cases. 


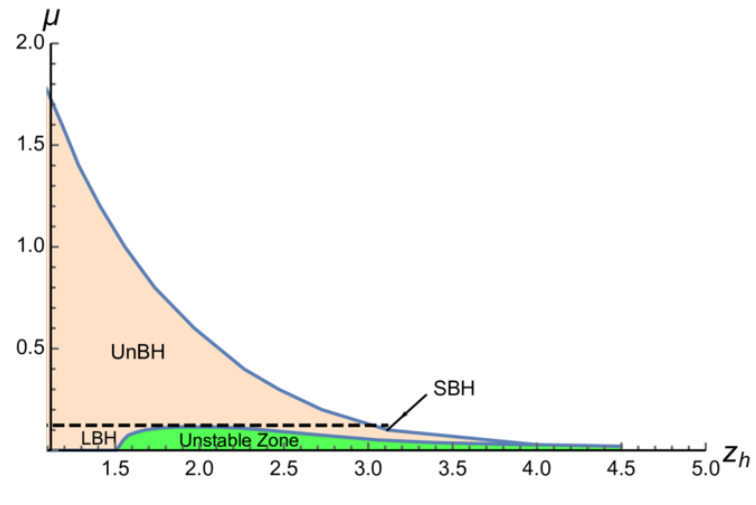

A

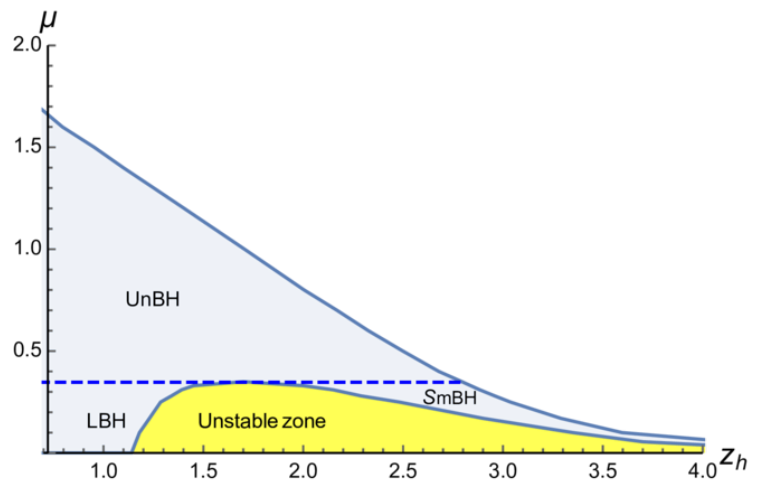

$\mathrm{B}$

Figure 31. Stability zones at the $\left(z_{h}, \mu\right)$-plane of the blackhole isotropic (A) and anisotropic (B) backgrounds. Here SBH indicates the regions of small black holes, LBH indicates the regions of large black holes, and UnBH indicates the regions of parameters where is no separation on the small and large black holes.

extremal point of the effective potential, that depends on the orientation [35] and is related to the warp factor power $P(z)$ and the scalar field $\phi$ :

$$
\begin{aligned}
& \mathcal{V}_{x}=\frac{e^{P(z)+\sqrt{\frac{2}{3}} \phi(z)}}{z^{2}} \sqrt{g(z)}, \\
& \mathcal{V}_{y}=\frac{e^{P(z)+\sqrt{\frac{2}{3}} \phi(z)}}{z^{1 / \nu+1}} \sqrt{g(z)} .
\end{aligned}
$$

Here the subscribte indexes show the orientation of the Wilson loop. Therefore the dynamical wall position is given by equations:

$$
\begin{aligned}
& \mathcal{V}_{x}^{\prime}\left(z_{D W x}\right)=0 \Rightarrow P^{\prime}(z)+\sqrt{\frac{2}{3}} \phi^{\prime}(z)-\frac{2}{z}+\left.\frac{g^{\prime}(z)}{2 g(z)}\right|_{z=z_{D W x}}=0 \\
& \mathcal{V}_{y}^{\prime}\left(z_{D W y}\right)=0 \Rightarrow P^{\prime}(z)+\sqrt{\frac{2}{3}} \phi^{\prime}(z)-\frac{\nu+1}{\nu z}+\left.\frac{g^{\prime}(z)}{2 g(z)}\right|_{z=z_{D W y}}=0 .
\end{aligned}
$$

For zero temperature we have

$$
\begin{array}{r}
P^{\prime}(z)+\sqrt{\frac{2}{3}} \phi^{\prime}(z)-\left.\frac{2}{z}\right|_{z=z_{D W x}} 0, \\
P^{\prime}(z)+\sqrt{\frac{2}{3}} \phi^{\prime}(z)-\left.\frac{\nu+1}{\nu z}\right|_{z=z_{D W y}}=0 .
\end{array}
$$

Substituting $\phi^{\prime}(z)$ from (2.6) we get the following equations for the positions of the dynamical wall corresponding to $\mathrm{x}$ - and $\mathrm{y}$-directions of the quark orientations:

$$
\begin{gathered}
{\left.\left[P^{\prime}(z)+\sqrt{\frac{2}{3}} \sqrt{-3 P^{\prime \prime}(z)+\frac{3}{2} P^{\prime 2}(z)-\frac{6}{z} P^{\prime}(z)+\frac{4}{z^{2}} \frac{\nu-1}{\nu^{2}}}-\frac{2}{z}\right]\right|_{z=z_{D W x}}=0} \\
{\left.\left[P^{\prime}(z)+\sqrt{\frac{2}{3}} \sqrt{-3 P^{\prime \prime}(z)+\frac{3}{2} P^{\prime 2}(\xi)-\frac{6}{z} P^{\prime}(\xi)+\frac{4}{z^{2}} \frac{\nu-1}{\nu^{2}}}-\frac{\nu+1}{\nu z}\right]\right|_{z=z_{D W y}}=0}
\end{gathered}
$$




\section{$4.2 \quad P(z)=c z^{2} / 2$}

We take the simplest case (2.31) again. Therefore we choose the expression with positive sign in (2.56), and equations (4.3) and (4.4) become:

$$
\begin{aligned}
& \mathcal{D} \mathcal{W}_{x} \equiv c z+\frac{1}{\nu z} \sqrt{\frac{2}{3}} \sqrt{3 c \nu^{2} z^{2}\left(\frac{c z^{2}}{2}-3\right)+4 \nu-4}-\frac{2}{z}+\left.\frac{g^{\prime}}{2 g}\right|_{z=z_{D W x}}=0 \\
& \mathcal{D} \mathcal{W}_{y} \equiv c z+\frac{1}{\nu z} \sqrt{\frac{2}{3}} \sqrt{3 c \nu^{2} z^{2}\left(\frac{c z^{2}}{2}-3\right)+4 \nu-4}-\frac{\nu+1}{\nu z}+\left.\frac{g^{\prime}}{2 g}\right|_{z=z_{D W y}}=0 .
\end{aligned}
$$

Note that in both equations there is also a dependence of the corresponding blackening functions on $\nu$. The phase transition from confinement to deconfinement occurs when the corresponding equations loss solutions.

To see the dependence of the dynamical wall position on the parameters of the metric, it is useful to study the details of dependence of the different terms defined $\mathcal{D} \mathcal{W}_{x}, \mathcal{D W}_{y}$ and $\mathcal{D} \mathcal{W}_{\text {iso }}$ on these parameters. Here $\mathcal{D} \mathcal{W}_{\text {iso }}$ denotes the left-hand side of the equation, similar to (4.8), in the isotropic case.

\subsubsection{Zero temperature}

Let us first consider the case of the zero temperature, i.e. $g=1$. In this case we deal with equations

$$
\begin{aligned}
& z=z_{D W x}: \quad \sigma(z, \nu, c)=\frac{2}{z} \quad \text { or } \quad \sigma_{x}(z, \nu, c)=0, \\
& z=z_{D W y}: \quad \sigma(z, \nu, c)=\frac{\nu+1}{\nu z} \quad \text { or } \quad \sigma_{y}(z, \nu, c)=0 \text {, }
\end{aligned}
$$

where

$$
\sigma(z, c, \nu) \equiv c z+\frac{1}{\nu z} \sqrt{\frac{2}{3}} \sqrt{3 c \nu^{2} z^{2}\left(\frac{c z^{2}}{2}-3\right)+4 \nu-4} .
$$

We also use notations:

$$
\begin{aligned}
& \sigma_{x}(z, c, \nu) \equiv c z+\frac{1}{\nu z} \sqrt{\frac{2}{3}} \sqrt{3 c \nu^{2} z^{2}\left(\frac{c z^{2}}{2}-3\right)+4 \nu-4}-\frac{2}{z} \\
& \sigma_{y}(z, c, \nu) \equiv c z+\frac{1}{\nu z} \sqrt{\frac{2}{3}} \sqrt{3 c \nu^{2} z^{2}\left(\frac{c z^{2}}{2}-3\right)+4 \nu-4}-\frac{\nu+1}{\nu z} .
\end{aligned}
$$

Behavior of $\sigma_{x}(z, c, \nu)$ and $\sigma_{y}(z, c, \nu)$ as functions of $z$ are shown in figure 32. The positions of the dynamical walls are defined by the intersections of the solid blue and magenta lines representing $\sigma_{x}(z, c, \nu)$ and $\sigma_{y}(z, c, \nu)$ with the horizontal line $\sigma=0$.

To show that the presence of the dilaton field supports the appearance of the DW, we display parts of the expressions $\sigma_{x}(z, c, \nu)$ and $\sigma_{y}(z, c, \nu)$ without the square roots, that are originated from the dilaton fields, by the dashed lines in figure 32.B and C. We see that these dashed lines never intersect horizontal axis, therefore in these cases there are no DW solutions. 


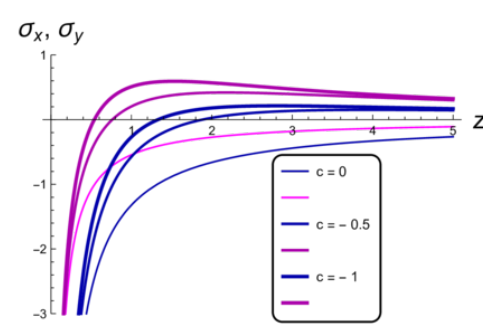

A

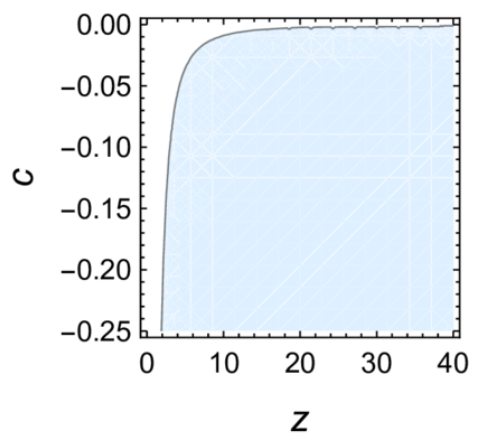

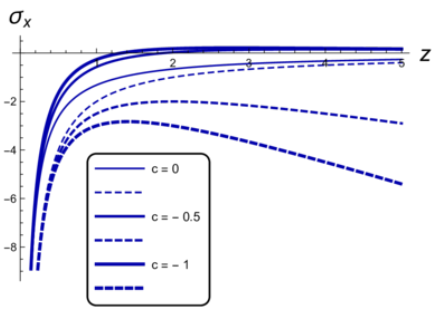

$\mathrm{B}$

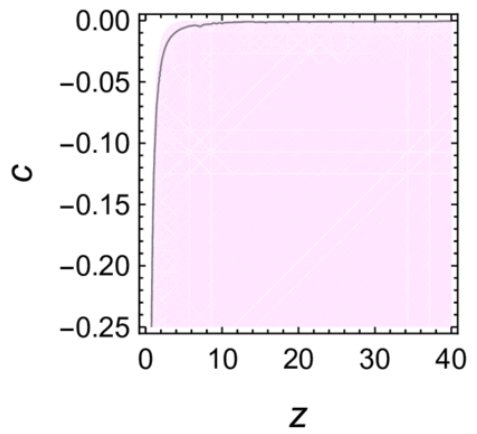

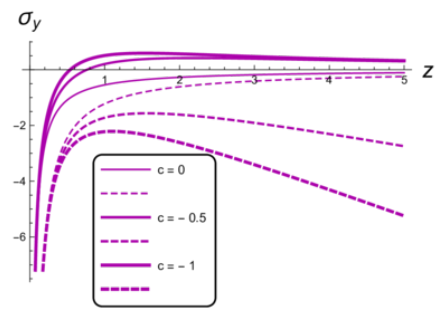

C

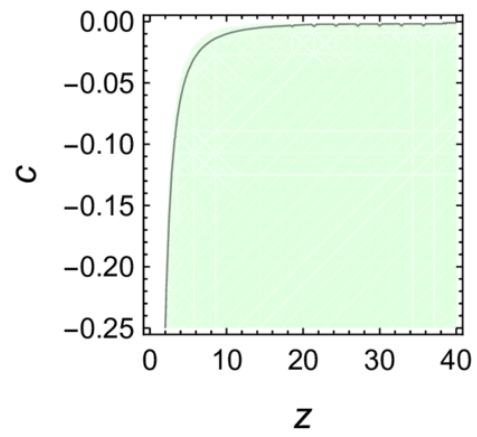

$\mathrm{D}$

Figure 32. Functions $\sigma_{x}(z, c, \nu)$ (blue lines) and $\sigma_{y}(z, c, \nu)$ (magenta lines) for different $c$ with dilaton contributions (solid lines) and without them (dashed lines) together (A) and separately (B, C); solutions to (4.9) (boundary of blue and white areas), to (4.10) (boundary of magenta and white areas) and for the isotropic case (boundary of green and white areas) (D).

Solutions to equations (4.9) and (4.10) can also be represented as the boundary between positive and negative values of functions $\sigma_{x}(z, c, \nu)$ and $\sigma_{y}(z, c, \nu)$. Taking $\nu=1$ provides us with the isotropic case result (figure 32.D). We see that the critical $c=c_{\mathrm{cr}}$, above which there are no solutions in all cases, is $c_{\mathrm{cr}}=0$. For $c>0$ our consideration is not valued, since the scalar field becomes complex.

\subsubsection{Non-zero temperature, zero chemical potential}

Let us take the nontrivial blackening function. The blackening function modifies the DW equations. It is convenient to present these equations in the form

$$
\begin{aligned}
& z=z_{D W x}: \quad \Sigma\left(z, z_{h}, \nu, c\right)=\frac{2}{z}, \\
& z=z_{D W y}: \quad \Sigma\left(z, z_{h}, \nu, c\right)=\frac{\nu+1}{\nu z}
\end{aligned}
$$

where

$$
\Sigma\left(z, z_{h}, c, \nu\right) \equiv \sigma(z, c, \nu)+G\left(z, z_{h}, c, \nu\right) .
$$

Function $\sigma(z, \nu, c)$ is given by (4.11) and

$$
G\left(z, z_{h}, c, \nu\right) \equiv \frac{g^{\prime}}{2 g} .
$$




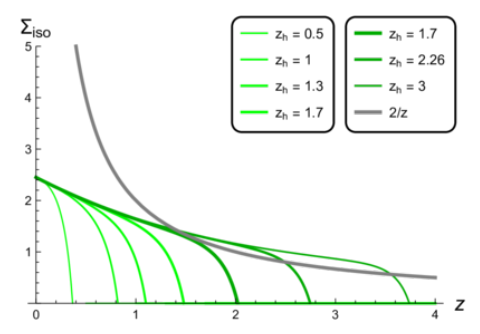

A

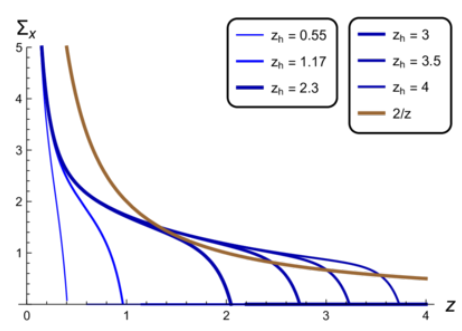

$\mathrm{B}$

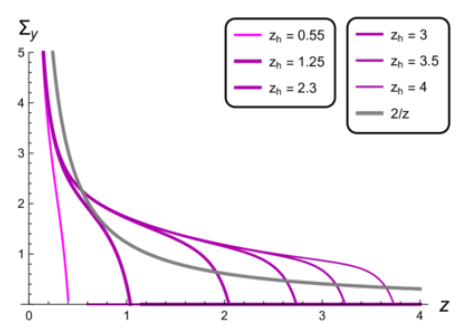

C

Figure 33. A) Dynamical walls' positions in isotropic case are given by the intersections of green lines representing $\Sigma_{\text {iso }}(z)$ and the grey line representing $2 / z ; \mathrm{B}$ ) dynamical walls' positions, corresponding to the Wilson loop $W_{x}$ in the anisotropic case $\nu=4.5$, are given by intersections of blue lines representing $\Sigma(z)$ and the brown line representing $2 / z$; C) dynamical walls' positions, corresponding to the Wilson loop $W_{y}$ in the anisotropic case $\nu=4.5$ are given by the intersections of magenta lines representing $\Sigma(z)$ and the grey line representing $(\nu+1) /(\nu z)$. Here we vary $z_{h}$ and $c$. In all cases to get the DW position we take the minimal intersection point.

We find the solutions of equations (4.14) and (4.15) numerically. To visualize the location of these solutions we plot $\Sigma\left(z, z_{h}, c, \nu\right)$ as a function of $z$ for different values of parameters $z_{h}, \nu, c$ and find its intersection with $2 / z$ for the Wilson loop $W_{x}$ and with $(\nu+1) /(\nu z)$ for the Wilson loop $W_{y}$ in figure 33. If there are two intersection points, we take the minimal one and we call it the minimal intersection point. In all cases to get the corresponding DW position we take the minimal intersection point. From figure 33.A we see that for $z_{h}>z_{h, \text { cr }}$ the dynamical wall always appears, as there are intersections of the grey and dark green lines. At the critical horizon (the thick dark green line) there is a touch of these two lines and for $z<z_{h, \text { cr }}$ (lighter green lines) there is no intersection at all, therefore confinement disappears.

The light blue curves in figure 33.B do not cross the grey line and for these cases there are no dynamical walls. The dark blue lines cross the brown one and for the corresponding temperature there is the quark confinement, meanwhile the thick dark blue line just touches the grey line and at this temperature the phase transition occurs. The similar picture can be seen at figure 33.C, corresponding to different orientation of quark pairs.

Therefore the dynamical wall always appears for $z_{h}>z_{h, \mathrm{cr}}$. The particular values of $z_{h, \mathrm{cr}}$ are different for isotropic (A) and anisotropic (B, C) cases and depend on the quark orientations. This appearance/disappearance of dynamical walls corresponds to confinement and deconfinement phases. The phase transition between these two regimes occurs at $z=z_{h, \mathrm{cr}}$.

In figure 34 solutions to equations (4.14), (4.15) are located on the boundary of the colored and white areas. Since to find the dynamical walls' positions we have to take the minimal solutions, the dynamical walls' positions are located on the left parts of boundaries between the colored and white areas. 


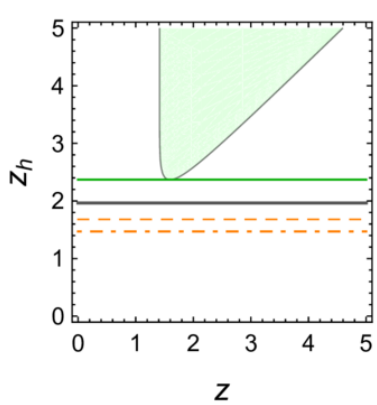

A

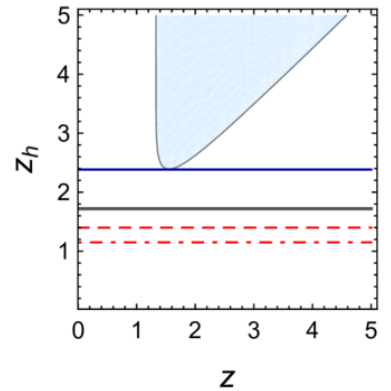

$\mathrm{B}$

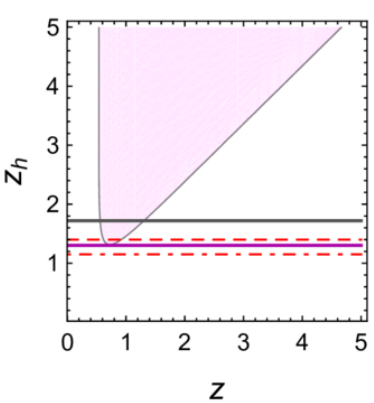

C

Figure 34. A) Dynamical walls' positions in isotropic case are located on the left part of the boundary of the green and white areas. We see that for given $z_{h}>2.304$ there are two solutions and for $z_{h}=2.304$ there is only one $z_{D W i s o}=1.455$. B) Dynamical walls' positions corresponding to the Wilson loop $W_{x}$ are located on the left part of the boundary of the blue and white ares. We see that for given $z_{h}>2.376$ there are two solutions and for $z_{h}=2.376$ only one $z_{D W x}=1.503$. C) Dynamical walls' positions corresponding to the Wilson loop $W_{y}$ are located on the left part of the boundary of the magenta and white ares. We see that for given $z_{h}>1.326$ there are two solutions and for $z_{h}=1.326$ only one $z_{D W i s o}=0.6871$. Here $\nu=4.5$ and $c=-1$. Dashed orange and red lines show positions of the critical $z_{h, \min }(0)$ in the corresponding isotropic and anisotropic backgrounds, and point-dashed lines show positions of the horizons $z_{h, H P}(0)$, where the HP transitions take place at zero chemical potential in the isotropic and anisotropic cases. The dark grey lines show $z_{h, \mathrm{cr}}=z_{h, \mathrm{cr}}\left(\mu_{\mathrm{cr}}\right)$ the value of the horison at the inflection points. These plots show that all dynamical walls appear below the corresponding horizons, i.e. $z_{D W}\left(z_{h}, \nu\right)<z_{h}$ and these horizons $z_{h}$ are larger than $z_{h \text {,min }}(0)$ and $z_{h, \text { cr }}$ for the isotropic case and for $W_{x}$ case, but they can be smaller than $z_{h, \min }(0)$ and $z_{h, \mathrm{cr}}$ in the anisotropic $W_{y}$ case. The positions of minimal $z_{h}$ admitted the DW, $z_{h, D W}$ are indicated by dark green, dark blue and dark magenta lines.

\subsubsection{Non-zero chemical potential}

We can also study how these plots look for non-zero chemical potential. The positions of the dynamical walls for non-zero temperature and non-zero chemical potential in isotropic and anisotropic cases are presented in figure 35.A and figure 35.B correspondingly.

It is convenient to write equations (4.9) and (4.10) in the form

$$
\begin{aligned}
& z=z_{D W x}: \quad \Sigma\left(z, z_{h}, \mu, c, \nu\right)=\frac{2}{z} \\
& z=z_{D W y}: \quad \Sigma\left(z, z_{h}, \mu, c, \nu\right)=\frac{\nu+1}{\nu z}
\end{aligned}
$$

where

$$
\begin{aligned}
\Sigma\left(z, z_{h}, \mu, c, \nu\right) & \equiv \sigma(z, c, \nu)+G\left(z, z_{h}, \mu, c, \nu\right), \\
G\left(z, z_{h}, \mu, c, \nu\right) & \equiv \frac{g^{\prime}}{2 g}
\end{aligned}
$$

and $\sigma(z, c, \nu)$ is defined by (4.11).

The light blue and dark blue curves in figure 35.A represent the function $G\left(z, z_{h}, \mu, c, \nu\right)$ for $\nu=4.5, c=-1, z_{h}=1.5$ and different $\mu$. The light magenta and dark magenta 


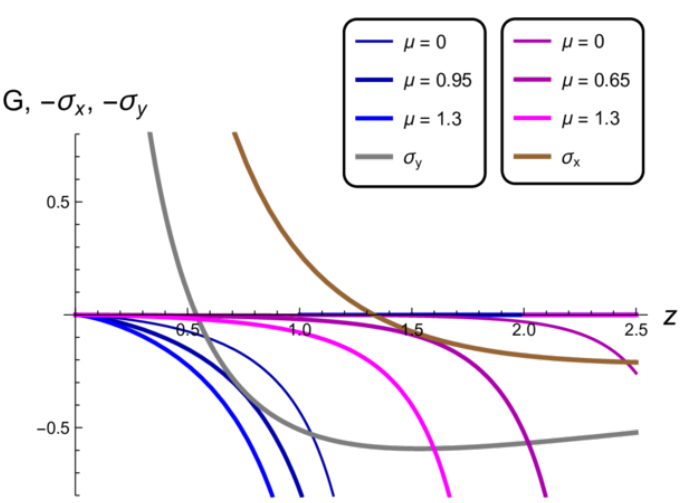

A

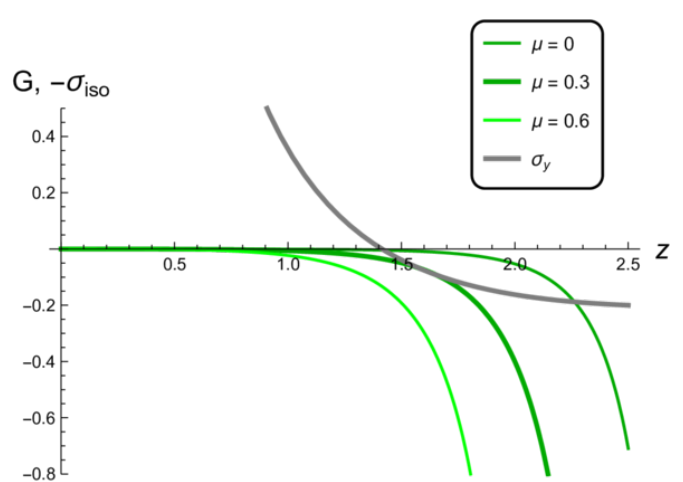

$\mathrm{B}$

Figure 35. The positions of the dynamical walls $G=-\sigma_{x}$ for $z_{h}=1.5$ and $G=-\sigma_{y}$ for $z_{h}=3$ in anisotropic $\nu=4.5(\mathrm{~A})$ and isotropic $G=-\sigma_{\text {iso }}(z)$ (B) cases, $c=-1$.

lines correspond to $G\left(z, z_{h}, \mu, c, \nu\right)$ for the same set of parameters and $z_{h}=3$. The thick lines, that touch $\sigma_{x}$ (grey line) and $\sigma_{y}$ (brown line), depict the critical values of chemical potential $\mu$. Thus the presence and the particular position of the horizon modifies the position of dynamical walls as compare with the zero temperature case $(g=1)$ presented in figure 32. Anisotropy also influences on the dynamical walls' position. This can be seen from comparing of figure 35.A and figure 35.B, where the isotropic case is pictured.

To find the phase transition line we have to determine $\mu\left(z_{h}, c, \nu, W_{i}\right)$, here $W_{i}$ indicates the orientation of the Wilson line, $W_{x}$ or $W_{y}$, for given $z_{h}$ such that for any $\mu>\mu\left(z_{h}, c, \nu, W_{i}\right)$ there is no real solution of equations (4.7) and (4.19). To find these points, it is convenient to draw the contour plots for functions $\mathcal{D} \mathcal{W}_{x}$ and $\mathcal{D} \mathcal{W}_{y}$ near zero. They are presented in figure 36 and figure 37 correspondingly. For comparison we present in figure 38 the contour plots for $\mathcal{D} \mathcal{W}_{\text {iso }}$ near 0.

\subsubsection{The dynamical wall position}

All previous considerations can be summarized in contour plots. Namely, we can draw the contours for the locations of the effective potentials' derivatives $\mathcal{V}_{x y}^{\prime}=0$ in the $\left(z, z_{h}\right)$-plane, keeping $c=-1$ and considering two cases $\nu=1$ (figure 39) and $\nu=4.5$ (figure 40). Different contours correspond to different values of $\mu$. Dynamical walls' positions correspond to the minimal $z$ for fixed horizon $z_{h}$ and chemical potential $\mu$ (figure 39.A). The positions of the dynamical walls in the anisotropic case differ for longitudinal $x$ and transversal $y_{1,2}$ quark pair orientation (figure 40.A and C, correspondingly). Transversal case is characterized by smaller values of $z_{h}$ and minimal $z$ for the same chemical potential $\mu$.

On figure 39.B and figure 40.B and D the instability regions of the background for $\mu=0$ are indicated by black arrows. For fixed $0<\mu<\mu_{\mathrm{cr}}$ they are shown as domains between corresponding brown solid and dashed lines of the same thickness. The thick magenta line on figure 40.D shows the value of $z_{h}$, at which the transition between large and small black holes disappears and the "removed zone" shrinks to $z_{h, \mathrm{cr}}$ for the curve $\mu=\mu_{\mathrm{cr}}$. For isotropic case $\mu_{\mathrm{cr}}^{(\mathrm{iso})}=0.119$ and for anisotropic one at $\mu_{\mathrm{cr}}^{(\nu)}=0.34$ and it 

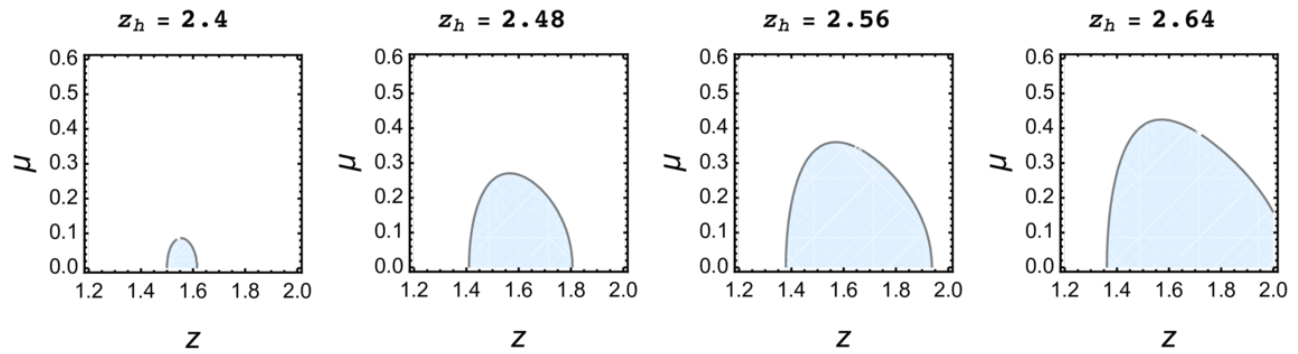

Figure 36. Contour plots for $\mathcal{D} \mathcal{W}_{x}$ near zero for different $z_{h}$ (labels above).
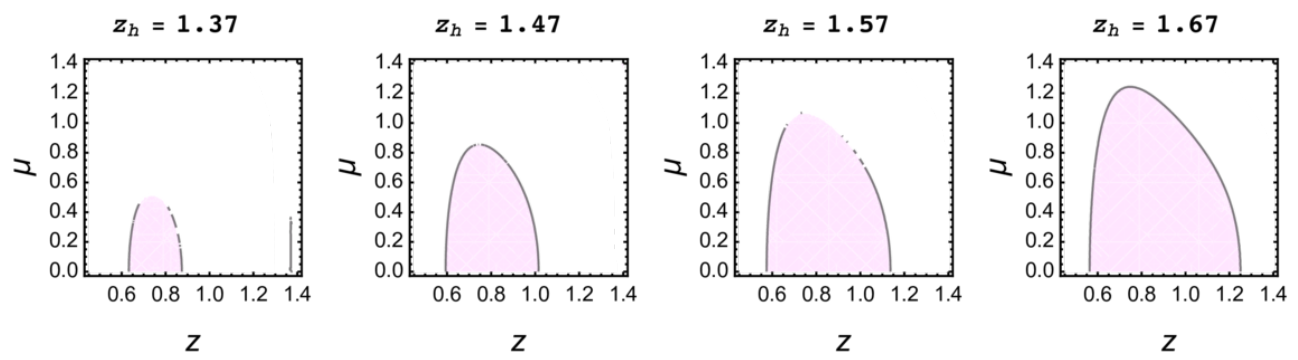

Figure 37. Contour plots for $\mathcal{D} \mathcal{W}_{y}$ near zero for different $z_{h}$ (labels above).
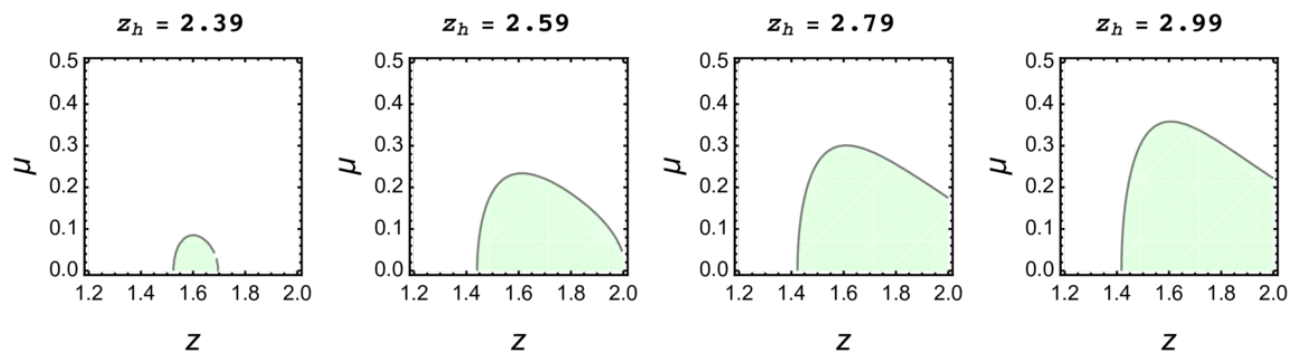

Figure 38. Contour plots for $\mathcal{D} \mathcal{W}_{\text {iso }}$ near zero value.

happens at $z_{h}=z_{h, \mathrm{cr}}^{\text {(iso) }}=1.97$ and at $z_{h}=z_{h, \mathrm{cr}}^{\text {(aniso) }}=1.70$ in the isotropic and anisotropic cases, respectively. Note, the line $z_{h}=z_{h, \mathrm{cr}}^{\text {(iso) }}$ is below $z_{h, D W}^{\text {(iso) }}$, the value of horizon for which the DW can appear in the isotropic case. In the anisotropic case the line $z_{h}=z_{h, \text { cr }}^{\text {(aniso) is }}$ below values of $z_{h, D W x}$, for which the DW for $W_{x}$ can appear, $z_{h, \mathrm{cr}}^{\text {(aniso }}<z_{h, D W x}$, but above the values of horizons, for which the DW can appear for $W_{y}$, i.e. $z_{h, \text { cr }}^{(\text {aniso })}>z_{h, D W y}$ (see figure 41 and discussion below).

\subsubsection{Phase transition lines and critical points}

Confinement/deconfinement phase transition for the isotropic case $\nu=1$ is shown on figure 42. Note that for zero chemical potential the Hawking-Page temperature is less than the temperature of the confinement/deconfinement transition temperatute, $T_{H P}(0)<$ $T_{C D}(0)$. The temperature of the black hole to black hole transition $T_{B B}(\mu)$ is less than the temperature of the confinement/deconfinement transition $T_{C D}(\mu)$ for $0<\mu<\mu_{b}^{(\text {iso })}$, i.e. 


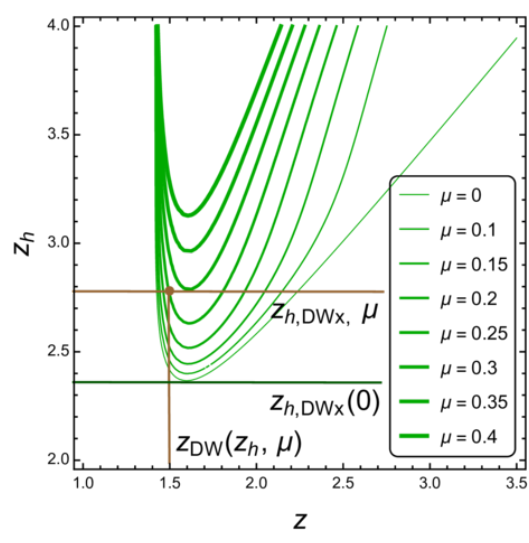

$\mathrm{A}$

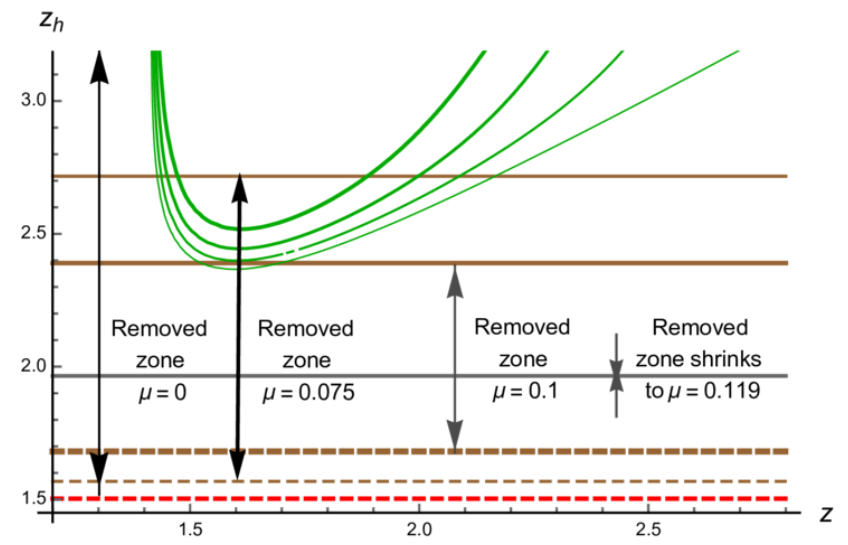

B

Figure 39. A) The location of extreme points of the effective potential in the isotropic case $\nu=1$, $c=-1$ for different $z_{h}$ and $\mu$ are shown by green lines with different thickness, depending on the values of the chemical potential. Dynamical walls' positions are located on the left parts of these curves. B) Locations of instability zones corresponding to different $\mu$ are shown by the black lines with arrows.

$T_{B B}(\mu)<T_{C D}(\mu)$, and $T_{B B}(\mu)>T_{C D}(\mu)$ for $\mu_{b}^{\text {(iso) }}<\mu<\mu_{\mathrm{cr}}^{\text {(iso) }}$ (let us remind that the background transition line stops at $\left.\left(\mu_{\mathrm{cr}}^{\text {(iso) }}, T_{\mathrm{cr}}^{(\text {iso })}\right)\right)$. Therefore, the phase transition line for $0<\mu<\mu_{b}^{\text {(iso) }}$ is determined by the background transition line (the orange lines at figure 42) and for $\mu \geq \mu_{b}^{\text {(iso) }}$ by the isotropic confinement/deconfinement transition line (the green lines at figure 42). This is in agreement with results of the previous studies [46] and refs. therein, where it has been argued that the transition for $\mu<\mu_{b}^{\text {(iso) }}$ is the first-order phase transition (FOPT) and for $\mu>\mu_{b}^{\text {(iso) }}$ is a smooth one.

The plots in figure 41 explain the difference between phase diagrams of Wilson lines $W_{x}$ and $W_{y}$ for $\nu=4.5$. In this plot we see that for $\mu=0$ the minimal value of the horizon $z_{h, D W x}$, for which the DW can appears for $W_{x}$, corresponds to small black hole, meanwhile the same horizon for $W_{y}, z_{h, D W y}$, corresponds to large black hole. By this reason we have

$$
T_{D W y}(0)<T_{H P}(0)<T_{D W x}(0) .
$$

Explicit numerical calculations show that

$$
T_{C D y}(\mu)<T_{B B}(\mu)<T_{C D x}(\mu) \text { for } 0<\mu<\mu_{x b}
$$

The confinement/deconfinement transition for the anisotropic case $\nu=4.5$ is shown on figure 43.A and B. The phase diagram for the longitudinal Wilson line $W_{x}$ is depicted by the blue lines, for transversal lines $W_{y}$ by the magenta lines and for the anisotropic background by the cyan lines. Wilson lines can also have arbitrary orientations, that corresponds to a modification of blue and magenta lines to some intermediate configuration.

The case $\nu=2$ can be considered as intermediate between the isotropic one and our main case $\nu=4.5$ (figure 43.C and D). Figure 44 combines all the three cases $\nu=1,2,4.5$ thus showing isotropization. 


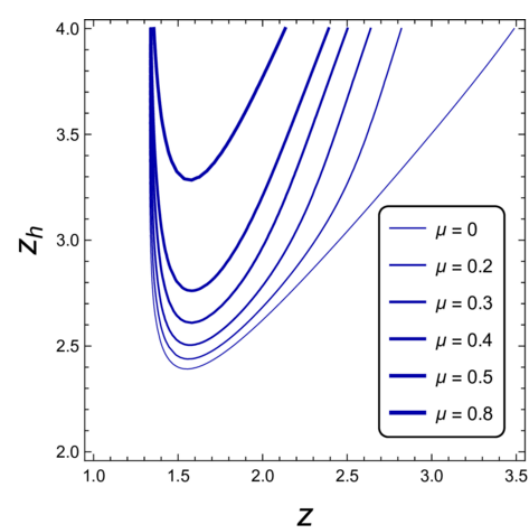

A

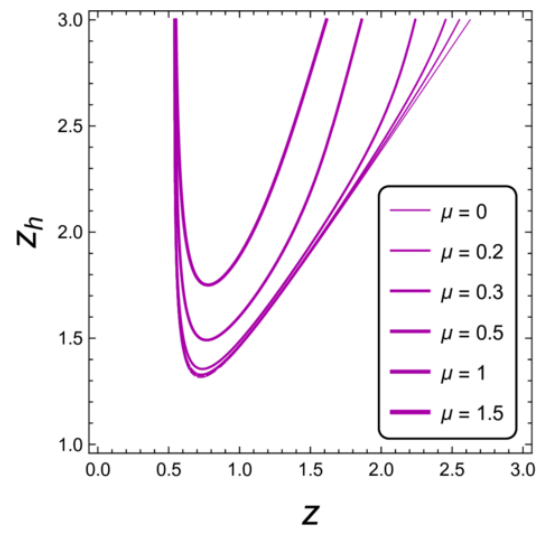

$\mathrm{C}$

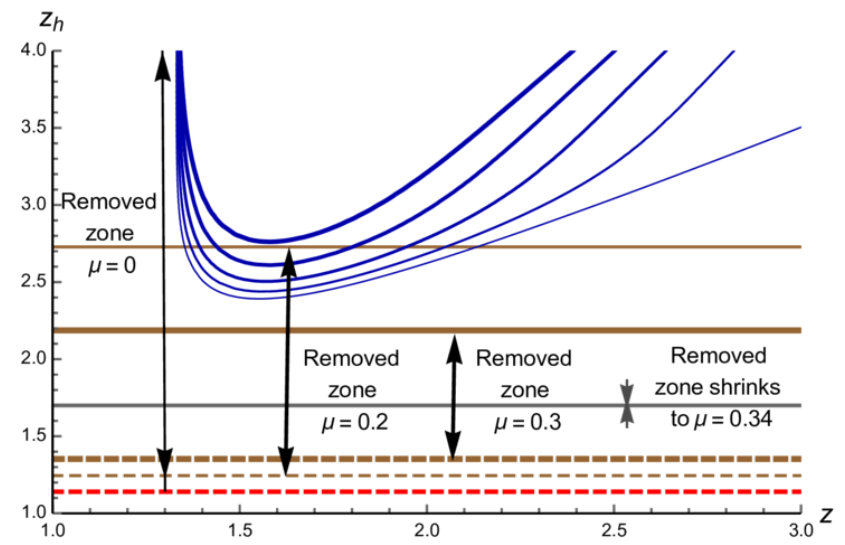

$\mathrm{B}$

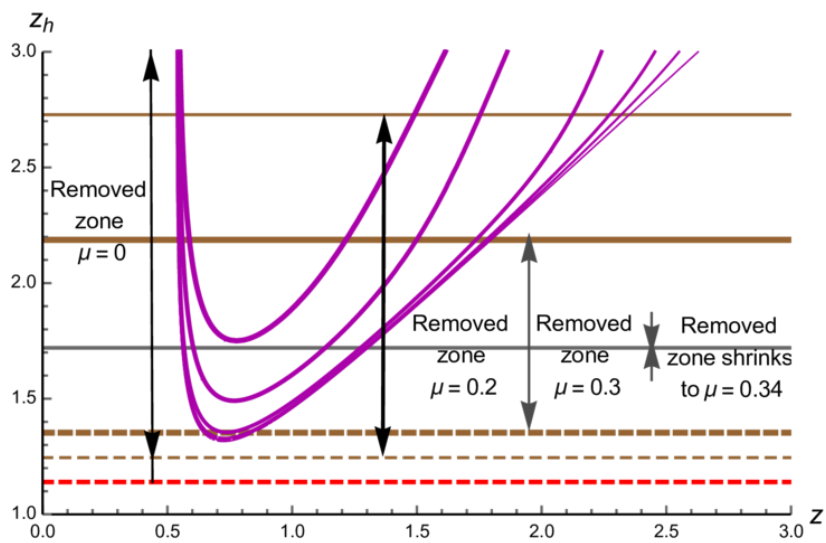

$\mathrm{D}$

Figure 40. The location of extreme points of effective potential in the anisotropic case $\nu=4.5$, $c=-1$ for different $z_{h}, \mu$ and different orientations of quark pairs are shown by lines of different thickness depending on the values of the chemical potential, and colors, for the Wilson longitudinal line $W_{x}\left(\mathrm{~A}\right.$ and $\mathrm{B}$ ) with dark blue and for the Wilson transverse line $W_{y}$ with dark purple lines (B and $\mathrm{D})$. B) and D). The location of the instability zones corresponding to different $\mu$ is shown by black lines with arrows.

\section{Conclusion and discussion}

We have considered 5-dimensional Einstein-dilaton-two-Maxwell-scalar system. We have found anisotropic solutions for this system by using the potential reconstruction method, i.e. choosing the corresponding dilaton and Maxwell potentials for the given background. This method has been used for isotropic cases in [45, 46, 49] and refs therein.

Our anisotropic background is the deformed $\mathrm{AdS}_{5}$ that has the UV boundary with two suppressed transversal coordinates and the IR boundary with the suppressed time and longitudinal coordinates. One can say that in this background two different 3-dimensional reductions, one in the UV domain and the other in the IR domain, are realized. The UV reduction is realized by the suppression of the original transversal coordinates, meanwhile 


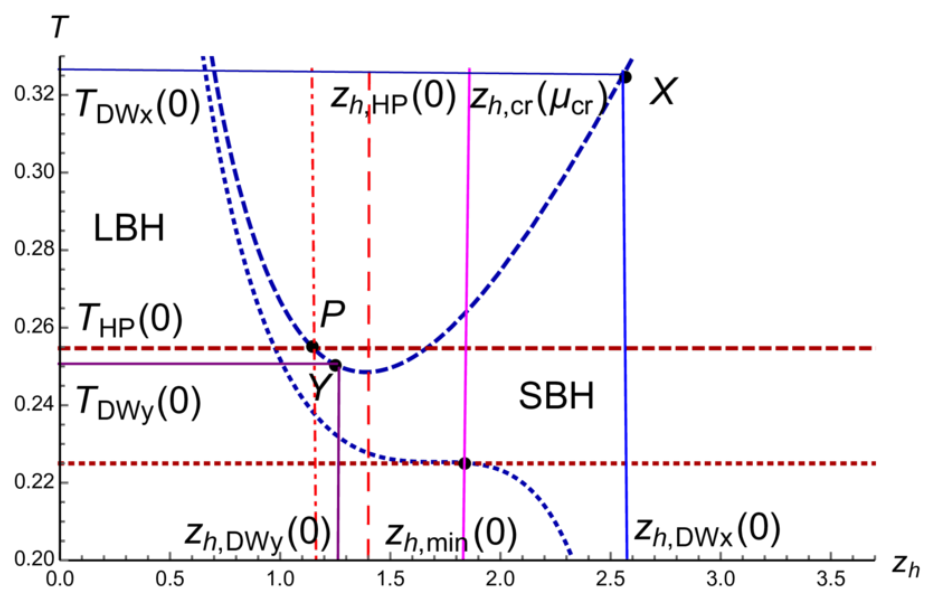

Figure 41. The plot explains the difference between phase diagrams for Wilson lines $W_{x}$ and $W_{y}$ for $\nu=4.5$. The coordinates of points $\mathrm{X}, \mathrm{Y}$ and $\mathrm{P}$ are $\left(z_{h, D W x}(0), T_{D W x}(0)\right),\left(z_{h, H P}(0), T_{H P}(0)\right)$ and $\left(z_{h, D W x}(0), T_{D W x}(0)\right)$. We see that $\mathrm{P}$ is in the SBH region, and $\mathrm{X}$ in the $\mathrm{LBH}$ region. The red dashed and point-dashed lines, and the magenta line are the same as at figure 34 .
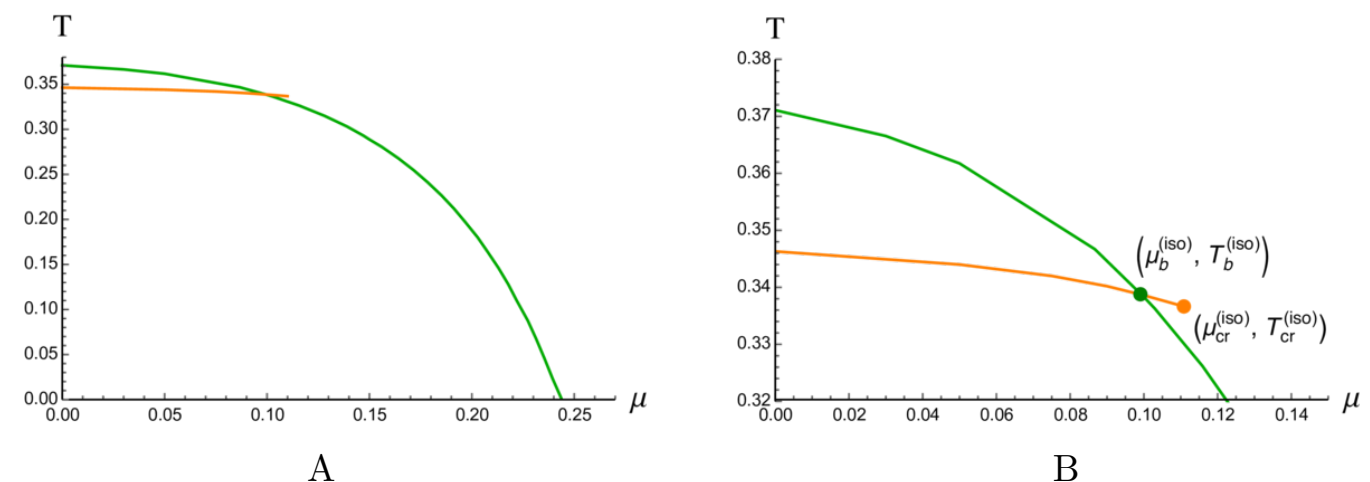

Figure 42. Confinement-deconfinement phase transition in the isotropic case, $\nu=1, c=-1$ for the Wilson line $W_{\text {iso }}$ (green) and the background (orange) (A). Plot (B) is a zoom of (A) near the intersection point $\left(\mu_{b}^{(\text {iso) }}, T_{b}^{\text {(iso) }}\right)$ and critical point $\left(\mu_{\mathrm{cr}}^{\text {(iso) }}, T_{\mathrm{cr}}^{\text {(iso) }}\right)$.

the IR reduction is obtained by the suppression of the longitudinal and temporal coordinates. For the corresponding isotropic solution there is no 3-dim reduction neither in UV nor in IR regions.

In our calculations the warped factor is chosen in such a way that the explicit analytical calculations can be performed. This solution can be generalized to provide a more realistic model. In this case, similar to the isotropic case, the solution can be given only in terms of quadratures. We solved the equations of motion to obtain a family of the black hole solutions by modifying the initial potential corresponding to zero temperature. In this construction the special boundary conditions for the dilaton field are chosen, namely we have required that the dilaton field is zero at the horizon.

We have also studied the thermodynamical properties of the constructed black hole background and found the large/small black hole phase transitions at the temperature 

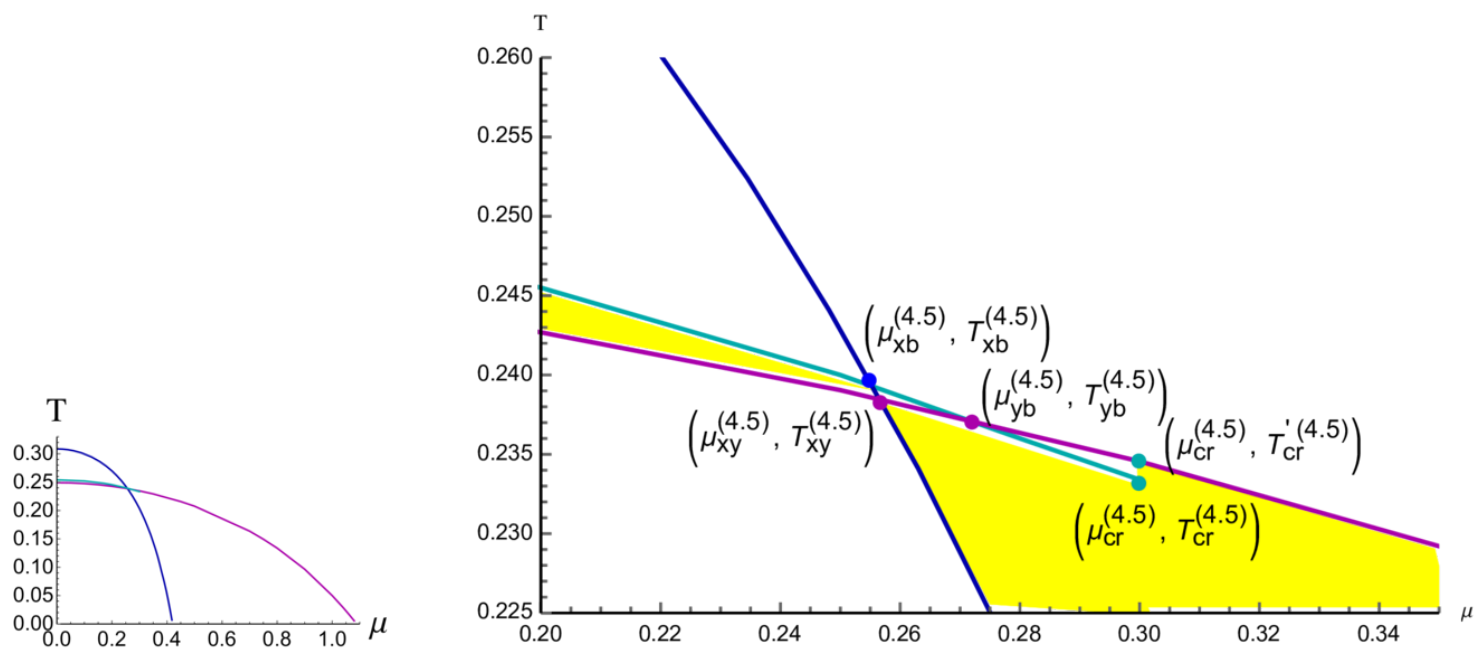

A

$\mathrm{B}$
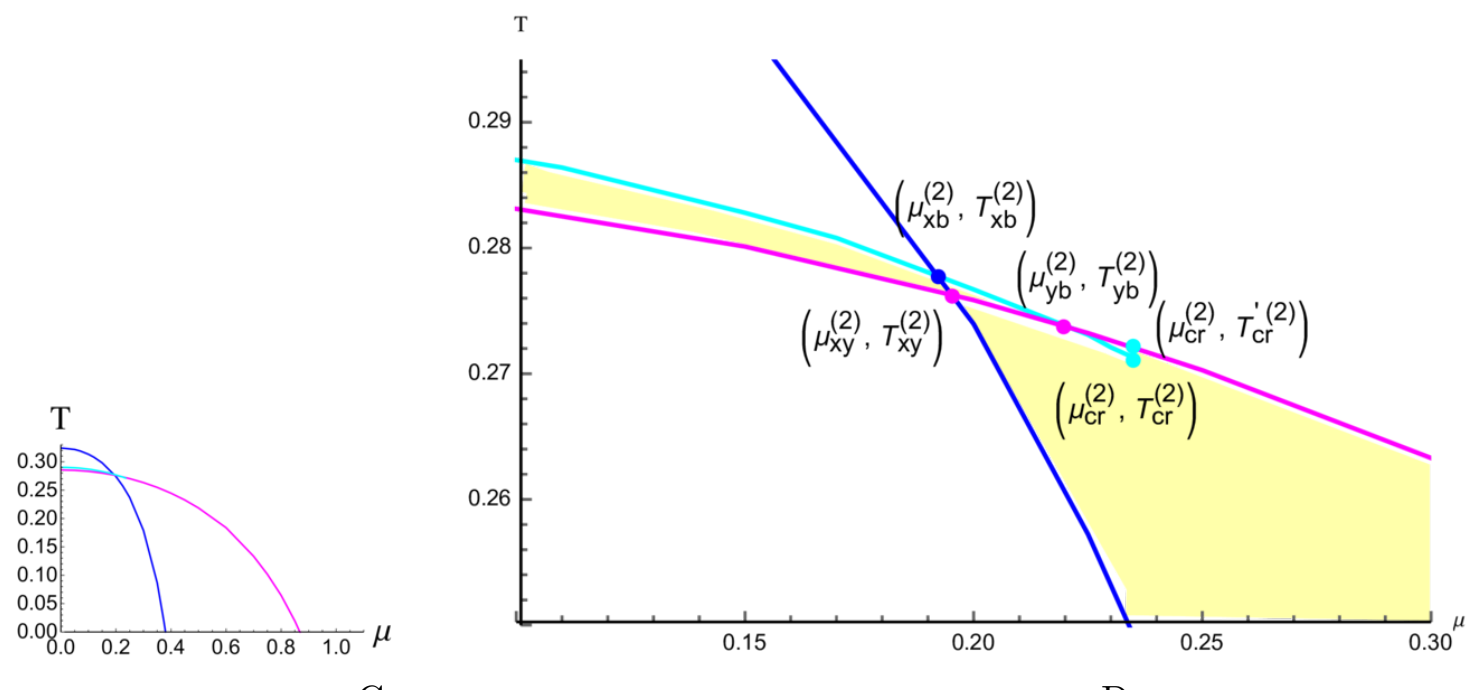

$\mathrm{C}$

$\mathrm{D}$

Figure 43. Confinement-deconfinement phase transition in the anisotropic case, $\nu=4.5, c=-1$, for the Wilson line $W_{x}$ (blue), the Wilson line $W_{y}$ (magenta) and the background (cyan) (A). Plot (B) is a zoom of (A) near the critical points $\left(\mu_{x b}, T_{x b}\right),\left(\mu_{y b}, T_{y b}\right)$ and FOPT $\left(\mu_{b}, T_{b}\right) \rightarrow\left(\mu_{b}, T_{b}^{\prime}\right)$. Plots (C) and (D) are the analogues of (A) and (B) for $\nu=2$.

magenta $T_{B B}(\mu)$. This result is presented in figure 29. At $\mu=0$ and for $T<T_{H P}(0)$, the black hole dissolves to thermal gas which is thermodynamically stable for $T<T_{H P}(0)$. When the system cools down with the chemical potential less than the critical value $\mu_{\mathrm{cr}}$, the background undergoes the phase transition from a large to a small black hole. This is a generalization of the corresponding effect in the isotropic case [45, 46, 50-52]. We have found that $T_{B B}^{(\text {aniso) }}(\mu)<T_{B B}^{\text {(iso) }}(\mu)$ and the value of the critical chemical potential, value up to which this phase transition exists, is bigger in the anisotropic case as the compare to the isotropic one, $\mu_{\mathrm{cr}}^{(\nu)}>\mu_{\mathrm{cr}}^{(\text {iso })}$, see figure 44 . Also, we have found that the point $\left(\mu_{\mathrm{cr}}^{(\nu)}, T_{\mathrm{cr}}^{(\nu)}\right)$ for $\nu \rightarrow 1$ goes smoothly to $\left(\mu_{\mathrm{cr}}^{\text {(iso) }}, T_{\mathrm{cr}}^{\text {(iso) }}\right)$. 


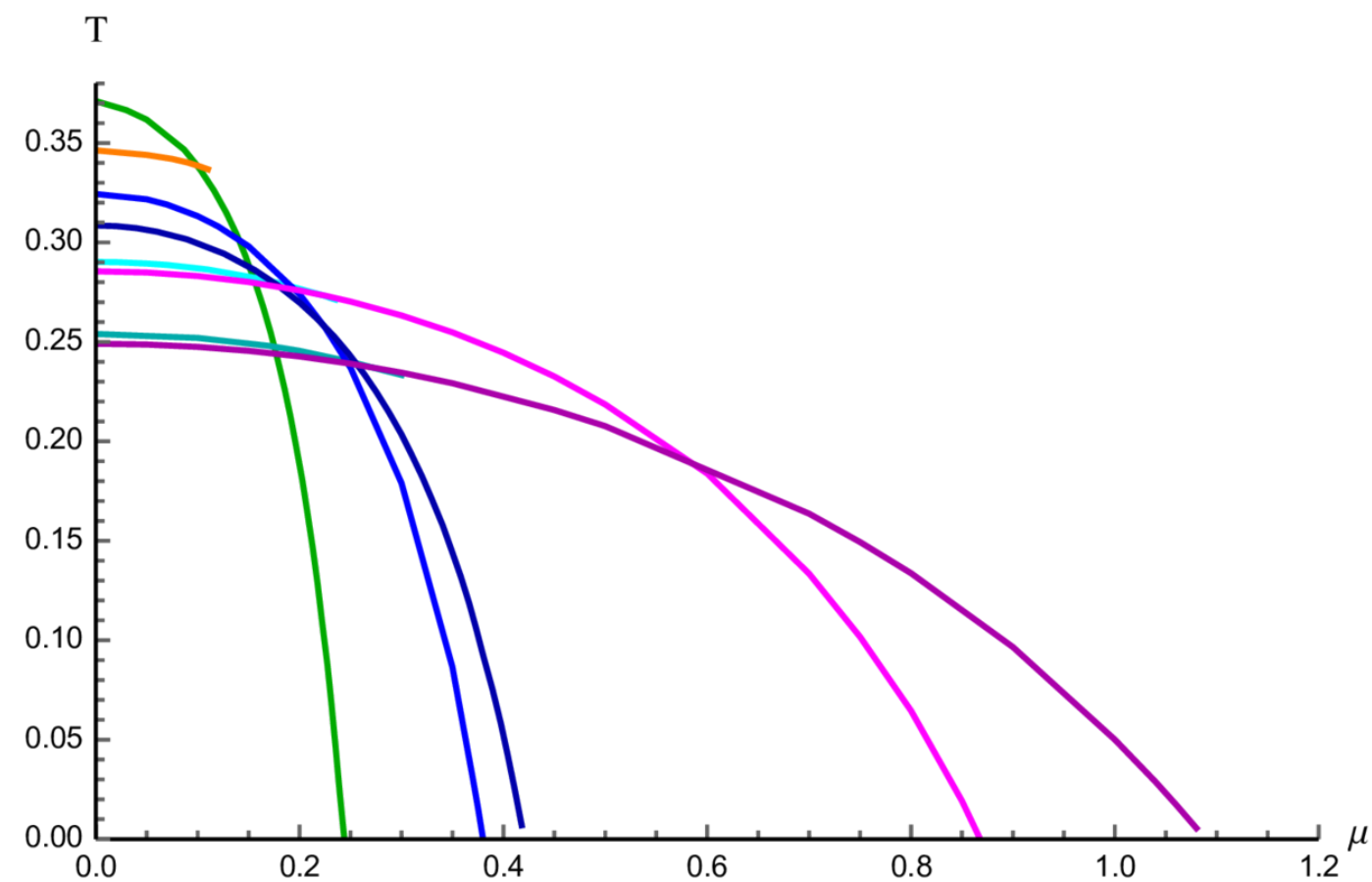

Figure 44. Confinement-deconfinement phase transitions of Wilson lines $W_{\text {iso }}$ for $\nu=1$ (green), $W_{x}$ and $W_{y}$ for $\nu=2$ (blue and magenta) and $\nu=4.5$ (dark blue and dark magenta); the background transition lines for $\nu=1$ (orange), $\nu=2$ (cyan) and $\nu=4.5$ (dark cyan).

We have studied the behavior of the temporal Wilson loops in the constructed background. For this purpose we have considered open strings in these backgrounds and identified the two ends of an open string as a quark and antiquark pair in the dual holographic QCD. As in the isotropic case, the equations of motion for this open string support two configurations - U-shape and straight-shape of the open strings. The U-shape for large distances between quarks provides the quark confinement and is realized in the presence of the dynamical wall (DW). We have found the domains in the $\left(z_{h}, \mu\right)$ planes, where the DW can appear for the longitudinal and transversal orientation of the temporal Wilson loops. In these regions the open strings cannot exceed the DW, even the separation of the quark and antiquark goes to infinite and the quark confinement takes place. We have found that the phase diagram depends on the orientation, cf. [35]. Taking into account the instability zones of the anisotropic background, we have found more complicated confinement/deconfinement phase diagrams for different oriented temporal Wilson loops and the details are the following:

- In the case of the longitudinal orientation, $W_{T x}$, parts of regions near zero values of the chemical potential, $\mu<\mu_{x b}$, enter to the instability regions of our background, where the small black holes collapse to large ones. Here the horizon suddenly blows up to pass the critical value $z_{h, D W x}(\mu)$ (see figure 18 and figure 40 , at the last plots these jumps are indicated by the arrows), so that the confinement phase transforms to the deconfinement one by a phase transition. While the chemical potential is greater than 
the critical value $\mu>\mu_{x b}$, the black hole horizon grows gradually and continuously passes the critical horizon, corresponding to $\left(\mu_{\mathrm{cr}}, T_{\mathrm{cr}}\right)$, so that the confinement phase transforms to the deconfinement phase smoothly. In other words, the confinementdeconfinement line is determined by the probe string behavior itself. It is worth to notice that the similar situation takes place in the isotropic case.

- In the case of the transversal orientation, $W_{y}$, situation is more interesting. It happens, that the background phase transition line for small $\mu, \mu<\mu_{y b}$, is located above the phase transition line for the Wilson line, and for small $\mu$ we have a smooth confinement-deconfinement phase transition. For $\mu_{y b}<\mu<\mu_{\text {cr }}$ we fall in the zone of instability of the background and the first order phase transition takes place.

As to the future investigations, the following natural questions to static and non-static properties of our model are worth noting. As to static properties, it is natural to

- investigate the opportunity to fix our holographic QCD model by a suitable choice of the function $P(z)$ in (2.16), so that in the isotropic limit it would fit the Cornell potential known by lattice QCD; it would be interesting to perform calculations on an anisotropic lattice and compare these future results with our model;

- consider more general anisotropic backgrounds and derive the corresponding anisotropic RG flows;

- study the Regge spectrum for mesons, adding the probe gauge fields to the backgrounds; we expect that similarly to the isotropic case, the gauge potential can be fixed requiring the linear Regge spectrum for mesons;

- consider estimations for direct photons;

- evaluate transport coefficients and their dependence on the anisotropy.

As to the thermalization processes, which are the main motivations of our considered of the anisotropic background, we suppose to reexamine

- the shock wave collisions in the anisotropic background with the warped factor;

- thermalization times for 2-point correlators; for the no-dilaton case this question has been addressed in [31, 34];

- time dependence of the transport coefficients, for the no-dilaton model see [35].

In this paper we have studied a particular anisotropic model specified by the anisotropy parameter $\nu$ and in all plots we take $\nu=4.5$, since just this case reproduces the total multiplicity dependence on energy, $\mathcal{M} \sim s^{0.155}$. It would be interesting to find isotropization of our solution and it is natural to expect that in this case the both phase transition lines, the large/small black hole transition and the string confinement/deconfinement transition, will smoothly move to their isotropic parters. We leave these matters to future works. 


\section{Acknowledgments}

This work was presented at the Helmholtz International Summer School "Hadron Structure and Hadronic Matter, and Lattice QCD", 20.08.2017-2.09.2017, BLTP JINR, Dubna, Russia, the 9th mathematical physics meeting: "Summer School and Conference on Modern Mathematical Physics", 18.09.2017-23.09.2017, Belgrade, "IV Russian-Spanish Congress Particle and Nuclear Physics at all Scales and Cosmology", 4.09.2017-8.09,2017, Dubna.

\section{A Equations of motion}

$00: \quad b^{\prime \prime}-\frac{\left(b^{\prime}\right)^{2}}{2 b}+b^{\prime}\left(\frac{g^{\prime}}{2 g}-\frac{2}{\nu z}\right)+\frac{2 b}{z^{2}}\left(\frac{1}{3}+\frac{2}{3 \nu}+\frac{1}{\nu^{2}}\right)-\frac{b g^{\prime}}{3 z g}\left(1+\frac{2}{\nu}\right)+\frac{b}{6}\left(\phi^{\prime}\right)^{2}+\frac{b^{2} V}{3 z^{2} g}+$

$$
+\frac{z^{2} f_{1}\left(A_{t}^{\prime}\right)^{2}}{6 g}+\frac{q^{2} z^{-2+\frac{4}{\nu}} f_{2}}{6 g}=0
$$

11: $\quad g^{\prime \prime}+\frac{3 g b^{\prime \prime}}{b}-\frac{3 g\left(b^{\prime}\right)^{2}}{2 b^{2}}-\frac{6 g b^{\prime}}{b \nu z}-g^{\prime}\left(\frac{2}{z}+\frac{4}{\nu z}-\frac{3 b^{\prime}}{b}\right)+\frac{2 g}{z^{2}}\left(1+\frac{2}{\nu}+\frac{3}{\nu^{2}}\right)+\frac{g}{2}\left(\phi^{\prime}\right)^{2}+\frac{b V}{z^{2}}+$

$$
-\frac{z^{2} f_{1}\left(A_{t}^{\prime}\right)^{2}}{2 b}+\frac{q^{2} z^{-2+\frac{4}{\nu}} f_{2}}{2 b}=0
$$

$22: \quad g^{\prime \prime}+\frac{3 g b^{\prime \prime}}{b}-\frac{3 g\left(b^{\prime}\right)^{2}}{2 b^{2}}-3 \frac{g b^{\prime}}{z b}\left(1+\frac{1}{\nu}\right)-g^{\prime}\left(\frac{4}{z}+\frac{2}{\nu z}-\frac{3 b^{\prime}}{b}\right)+\frac{2 g}{z^{2}}\left(3+\frac{2}{\nu}+\frac{1}{\nu^{2}}\right)+\frac{g}{2}\left(\phi^{\prime}\right)^{2}+$

$$
+\frac{b V}{z^{2}}-\frac{z^{2} f_{1}\left(A_{t}^{\prime}\right)^{2}}{2 b}-\frac{q^{2} z^{-2+\frac{4}{\nu}} f_{2}}{2 b}=0
$$

44: $\quad-\frac{6\left(b^{\prime}\right)^{2}}{b^{2}}+\frac{12 b^{\prime}}{z b}\left(1+\frac{1}{\nu}\right)+\frac{g^{\prime}}{g}\left(\frac{2}{z}+\frac{4}{\nu z}-\frac{3 b^{\prime}}{b}\right)-\frac{4}{z^{2}}\left(1+\frac{4}{\nu}+\frac{1}{\nu^{2}}\right)+\left(\phi^{\prime}\right)^{2}-\frac{2 b V}{z^{2} g}-$

$$
-\frac{z^{2} f_{1}\left(A_{t}^{\prime}\right)^{2}}{b g}-\frac{q^{2} z^{-2+\frac{4}{\nu}} f_{2}}{b g}=0
$$

where ${ }^{\prime}=\partial / \partial z$.

These equations can be transformed to the following equations

$$
\begin{array}{r}
g^{\prime \prime}+g^{\prime}\left(\frac{3 b^{\prime}}{2 b}-\frac{1}{z}-\frac{2}{\nu z}\right)-\frac{z^{2}}{b} f_{1}\left(A_{t}^{\prime}\right)^{2}=0, \\
2 g^{\prime}\left(1-\frac{1}{\nu}\right)+g\left(1-\frac{1}{\nu}\right)\left(\frac{3 b^{\prime}}{b}-\frac{4}{z}-\frac{4}{\nu z}\right)+\frac{q^{2} z^{-1+\frac{4}{\nu}} f_{2}}{b}=0 \\
b^{\prime \prime}-\frac{3\left(b^{\prime}\right)^{2}}{2 b}+\frac{2 b^{\prime}}{z}-\frac{4 b}{3 \nu z^{2}}\left(1-\frac{1}{\nu}\right)+\frac{b}{3}\left(\phi^{\prime}\right)^{2}=0 \\
\frac{b^{\prime \prime}}{b}+\frac{\left(b^{\prime}\right)^{2}}{2 b^{2}}+\frac{3 b^{\prime}}{b}\left(\frac{g^{\prime}}{2 g}-\frac{1}{z}-\frac{1}{\nu z}\right)-\frac{g^{\prime}}{3 z g} \frac{5 \nu+4}{\nu}+\frac{4\left(2 \nu^{2}+3 \nu+1\right)}{3 z^{2} \nu^{2}}+\frac{g^{\prime \prime}}{3 g}+\frac{2 b V}{3 z^{2} g}=0
\end{array}
$$


Substituting expressions for $g^{\prime \prime}$ from (A.2) and $b^{\prime \prime}$ from (A.4) into (A.5) we get the expression for $V$ without $g^{\prime \prime}$ and $b^{\prime \prime}$

$$
V=-\frac{z^{4} A_{t}^{\prime 2} f_{1}}{2 b^{2}}-\frac{3 z^{2} b^{\prime} g^{\prime}}{2 b^{2}}-\frac{3 z^{2} g b^{\prime 2}}{b^{3}}+\frac{9 z g b^{\prime}}{2 \nu b^{2}}+\frac{15 z g b^{\prime}}{2 b^{2}}+\frac{z g^{\prime}}{\nu b}+\frac{2 z g^{\prime}}{b}+\frac{z^{2} g \phi^{\prime 2}}{2 b}-\frac{8 g}{\nu b}-\frac{4 g}{b},
$$

that is nothing but the constraint equation.

One can check explicitly, that from eqs. (A.2)-(A.4) and (A.6) follows the field equation for the scalar field (2.9). Indeed, differentiating (A.6) we get

$$
\begin{aligned}
\frac{b}{z^{2} g} \frac{\partial V}{\partial \phi}= & \frac{1}{2 \nu z^{2} b^{3} g \phi^{\prime}} \\
& \left\{2 z b b^{\prime}\left(\nu z^{3} A_{t}^{\prime 2} f_{1}-3 g\left(2 \nu z b^{\prime \prime}+(7 \nu+3) b^{\prime}\right)\right)-\right. \\
& -b^{2}\left[z\left(\nu z\left(z^{2} A_{t}^{\prime 2} \phi^{\prime} f_{1}^{\prime}+3 b^{\prime \prime} g^{\prime}\right)+b^{\prime}\left(3 \nu z g^{\prime \prime}+(-5 \nu-7) g^{\prime}\right)\right)+\right. \\
& \left.+2 \nu z^{3} A_{t}^{\prime} f_{1}(\phi)\left(z A_{t}^{\prime \prime}+2 A_{t}^{\prime}\right)+g\left(b^{\prime}\left(-23 \nu+\nu z^{2} \phi^{2}-25\right)-3(5 \nu+3) z b^{\prime \prime}\right)\right]+ \\
& \left.+18 \nu z^{2} g b^{\prime 3}+b^{3}\left[2 z\left((2 \nu+1) g^{\prime \prime}+\nu g \phi^{\prime}\left(z \phi^{\prime \prime}+\phi^{\prime}\right)\right)+g^{\prime}\left(-4 \nu+\nu z^{2} \phi^{2}-14\right)\right]\right\} .
\end{aligned}
$$

Substituting in the R.H.S. of (A.7) $g^{\prime \prime}$ and $b^{\prime \prime}$ from (A.2) and (A.4), respectively, we get the following expression for $\frac{\partial V}{\partial \phi}$ without $g^{\prime \prime}$ and $b^{\prime \prime}$ :

$$
\begin{aligned}
\frac{b}{z^{2} g} & \frac{\partial V}{\partial \phi}=\frac{1}{4 \nu^{3} z^{3} b^{2} g \phi^{\prime}}\left\{2 \nu z b \left[\nu z\left(\nu z^{3} A_{t}^{\prime 2} \phi^{\prime} f_{1}^{\prime}+2(\nu-1) b^{\prime} g^{\prime}\right)+\right.\right. \\
& \left.+2(\nu-1) \nu z^{3} A_{t}^{\prime 2} f_{1}+g b^{\prime}\left(-7 \nu^{2}-9 \nu+3 \nu^{2} z^{2} \phi \prime 2+16\right)\right]+9(\nu-1) \nu^{2} z^{2} g b^{\prime 2}+ \\
& \left.+2 b^{2}\left[2 \nu z g^{\prime}\left(-4 \nu+\nu^{2} z^{2} \phi \prime 2+4\right)+g\left(4\left(5 \nu^{2}-2 \nu-3\right)+2 \nu^{3} z^{3} \phi^{\prime} \phi^{\prime \prime}-3(\nu+1) \nu^{2} z^{2} \phi \prime 2\right)\right]\right\} .
\end{aligned}
$$

In a similar way we get the expression for $f_{2}^{\prime}$ without $g^{\prime \prime}$ and $b^{\prime \prime}$. Indeed, we differentiate (A.3) and obtain

$$
\begin{aligned}
\frac{q^{2} z^{\frac{4}{\nu}}-2}{2 b g} \frac{\partial f_{2}}{\partial \phi}=\frac{q^{2} z^{\frac{4}{\nu}-2} f_{2}^{\prime}}{2 b g \phi^{\prime}}= & -\frac{(\nu-1)}{2 \nu^{3} z^{3} b g \phi^{\prime}}\left\{\nu z\left(5 \nu z b^{\prime} g^{\prime}-g\left[(\nu+16) b^{\prime}-3 \nu z b^{\prime \prime}\right)\right]+\right. \\
& \left.+2 b\left[\nu z\left(\nu z g^{\prime \prime}-(\nu+6) g^{\prime}\right)+8(\nu+1) g\right]\right\} .
\end{aligned}
$$

Then we substitute there (A.2) and (A.4) and get

$$
\begin{aligned}
\frac{q^{2} z^{\frac{4}{\nu}-2}}{2 b g} \frac{\partial f_{2}}{\partial \phi}= & \frac{(\nu-1)}{4 \nu^{3} z^{3} b^{2} g \phi^{\prime}}\left\{2 \nu z b\left(b^{\prime}\left((7 \nu+16) g-2 \nu z g^{\prime}\right)-2 \nu z^{3} A_{t^{\prime}} 2 f_{1}(\phi)\right)-\right. \\
& \left.-9 \nu^{2} z^{2} g b^{\prime 2}+2 b^{2}\left(8 \nu z g^{\prime}+g\left(-20 \nu+\nu^{2} z^{2} \phi^{\prime 2}-12\right)\right)\right\} .
\end{aligned}
$$

From (A.8) and (A.10) we get

$$
\frac{q^{2} z^{-2+\frac{4}{\nu}}}{8 b g} \frac{\partial f_{2}}{\partial \phi}+\frac{b}{z^{2} g} \frac{\partial V}{\partial \phi}=\phi^{\prime \prime}+\phi^{\prime}\left(\frac{g^{\prime}}{g}+\frac{3 b^{\prime}}{2 b}-\frac{\nu+2}{\nu z}\right)+\frac{z^{2}\left(A_{t}^{\prime}\right)^{2}}{2 b g} \frac{\partial f_{1}}{\partial \phi} .
$$

This is nothing but E.O.M. for the scalar field. 


\section{B Simplest solutions to system (2.5)-(2.10)}

\section{B.1 Solutions for anisotropic metric $\nu \neq 1$ and $c=0, A_{t} \neq 0$}

The system (2.5)-(2.10) for anisotropic metric $\nu \neq 1$ and $b(z)=1, A_{t} \neq 0$ has the form

$$
\begin{aligned}
g^{\prime \prime}+g^{\prime}\left(-\frac{1}{z}-\frac{2}{\nu z}\right)-z^{2} f_{1} A_{t}^{\prime 2} & =0, \\
-\frac{4}{\nu z^{2}}\left(1-\frac{1}{\nu}\right)+\phi^{\prime 2} & =0 \\
2 g^{\prime}\left(1-\frac{1}{\nu}\right)+g\left(1-\frac{1}{\nu}\right)\left(-\frac{4}{z}-\frac{4}{\nu z}\right)+q^{2} z^{-1+\frac{4}{\nu}} f_{2} & =0 \\
-V-\frac{z^{4}}{2} A_{t}^{\prime 2} f_{1}+\frac{z g^{\prime}}{\nu}+2 z g^{\prime}+\frac{z^{2} g \phi^{\prime 2}}{2}-\frac{8 g}{\nu}-4 g & =0 .
\end{aligned}
$$

Substituting $\phi^{\prime 2}$ from (B.2) into (B.4) we get

$$
-V-\frac{z^{4}}{2} A_{t}^{\prime 2} f_{1}+\frac{z g^{\prime}}{\nu}+2 z g^{\prime}-\frac{2 g}{\nu^{2}}-\frac{6 g}{\nu}-4 g=0
$$

The variation of the action (2.1) over scalar field $\phi$ and components $A_{\mu}^{(1)}$ of the first Maxwell fields' leads to the following EOM:

$$
\begin{aligned}
\phi^{\prime \prime}+\phi^{\prime}\left(\frac{g^{\prime}}{g}-\frac{\nu+2}{\nu z}\right)+\frac{z^{2} A_{t}^{\prime 2}}{2 g} \frac{\partial f_{1}}{\partial \phi}-\frac{q^{2} z^{-2+\frac{4}{\nu}}}{2 g} \frac{\partial f_{2}}{\partial \phi}-\frac{b}{z^{2} g} \frac{\partial V}{\partial \phi} & =0 \\
A_{t}^{\prime \prime}+A_{t}^{\prime}\left(\frac{f_{1}^{\prime}}{f_{1}}-\frac{2-\nu}{\nu z}\right) & =0 .
\end{aligned}
$$

The last equation (B.7) gives

$$
\log A_{t}^{\prime}=-\log f_{1}+\frac{2-\nu}{\nu} \log z+\log C_{1} \Rightarrow A_{t}^{\prime}=C_{1} \frac{z^{\frac{2-\nu}{\nu}}}{f_{1}} .
$$

If we take $f_{1}$ as in (2.17) we get

$$
\begin{aligned}
A_{t}^{\prime} & =C_{1} \frac{z^{\frac{2-\nu}{\nu}}}{z^{-2+\frac{2}{\nu}}}=C_{1} z, \\
A_{t}(z) & =\frac{C_{1}}{2} z^{2}+C_{2} .
\end{aligned}
$$

Taking into account

$$
\begin{aligned}
A_{t}(0) & =C_{2}=\mu, \\
A_{t}\left(z_{h}\right) & =\frac{C_{1}}{2} z_{h}^{2}+\mu=0,
\end{aligned}
$$

we get

$$
A_{t}(z)=\mu\left(1-z^{2} / z_{h}^{2}\right)
$$


Substituting this to the blacking function equation (B.1) we get

$$
g^{\prime \prime}+g^{\prime}\left(-\frac{1}{z}-\frac{2}{\nu z}\right)-4 \mu^{2} \frac{z^{2+\frac{2}{\nu}}}{z_{h}^{4}}=0 .
$$

From (B.2) we get for the scalar field

$$
\phi^{\prime}= \pm \sqrt{\frac{4}{\nu z^{2}}\left(1-\frac{1}{\nu}\right)}=\frac{2}{\nu z} \sqrt{\nu-1}
$$

and

$$
\phi=\frac{2}{\nu} \sqrt{\nu-1} \log z+C_{5}
$$

From (B.3) we get $f_{2}$

$$
f_{2}=-q^{2} z^{-1+\frac{4}{\nu}}\left[2 g^{\prime}\left(1-\frac{1}{\nu}\right)+g\left(1-\frac{1}{\nu}\right)\left(-\frac{4}{z}-\frac{4}{\nu z}\right)\right]
$$

and from (B.4) we get

$$
V=-\frac{z^{4}}{2} A_{t}^{\prime 2} f_{1}+\frac{z g^{\prime}}{\nu}+2 z g^{\prime}+\frac{z^{2} g \phi^{\prime 2}}{2}-\frac{8 g}{\nu}-4 g
$$

\section{B.2 Solutions for anisotropic metric $\nu \neq 1$ and $c=0, A_{t}=0$}

From the previous consideration it is easy to reproduce the solution has been found in [33].

From (2.5) we find

$$
g(z)=1-\frac{z^{2+\frac{2}{\nu}}}{z_{h}^{2+\frac{2}{\nu}}},
$$

Second, we substitute (B.19) into (2.6) and get

$$
f_{2}(z)=\frac{4 z^{-4 / \nu}}{q^{2}} \frac{(\nu-1)\left(1+3 \nu+2 \nu^{2}\right)}{\nu^{2}(1+2 \nu)},
$$

Then we solve (2.6):

$$
\phi(z)=C_{5} \pm 2 \frac{\sqrt{\nu-1}}{\nu} \log (z)
$$

Finally, we have to find $V$ from (2.8) and check the scalar equation (2.9). Substituting (B.19) into (2.8) we obtain

$$
V(z)=-2 \frac{(1+\nu)(1+2 \nu)}{\nu^{2}}
$$

Hence we get the solution given by (B.19), (B.20), (B.21) and (B.22), that coincides with [33]. 


\section{B.3 Vacuum solutions}

Without black brane, i.e. for $g=1$ the EOM (2.5)-(2.10) transform to:

$$
\begin{aligned}
\frac{z^{2}}{b} f_{1} A_{t}^{\prime 2} & =0, \\
b^{\prime \prime}-\frac{3\left(b^{\prime}\right)^{2}}{2 b}+\frac{2 b^{\prime}}{z}-\frac{4 b}{3 \nu z^{2}}\left(1-\frac{1}{\nu}\right)+\frac{b}{3}\left(\phi^{\prime}\right)^{2} & =0, \\
\left(1-\frac{1}{\nu}\right)\left(\frac{3 b^{\prime}}{b}-\frac{4}{z}-\frac{4}{\nu z}\right)+\frac{q^{2} z^{-1+\frac{4}{\nu}}}{b} f_{2} & =0, \\
-V-\frac{z^{4}}{2 b^{2}} A_{t}^{\prime 2} f_{1}-\frac{3 z^{2} b^{\prime 2}}{b^{3}}+\frac{9 z b^{\prime}}{2 \nu b^{2}}+\frac{15 z b^{\prime}}{2 b^{2}}+\frac{z^{2} \phi^{\prime 2}}{2 b}-\frac{8}{\nu b}-\frac{4}{b} & =0, \\
\phi^{\prime \prime}+\phi^{\prime}\left(\frac{3 b^{\prime}}{2 b}-\frac{\nu+2}{\nu z}\right)+\frac{z^{2} A_{t}^{\prime 2}}{2 b} \frac{\partial f_{1}}{\partial \phi}-\frac{q^{2} z^{-2+\frac{4}{\nu}}}{2 b} \frac{\partial f_{2}}{\partial \phi}-\frac{b}{z^{2}} \frac{\partial V}{\partial \phi} & =0, \\
A_{t}^{\prime \prime}+A_{t}^{\prime}\left(\frac{b^{\prime}}{2 b}+\frac{f_{1}^{\prime}}{f_{1}}-\frac{2-\nu}{\nu z}\right) & =0 .
\end{aligned}
$$

For $b=e^{\frac{c z^{2}}{2}}$ :

$$
\begin{aligned}
z^{2} e^{-\frac{c z^{2}}{2}} f_{1} A_{t}^{\prime 2} & =0 \\
\frac{c^{2} z^{2}}{2}-3 c+\frac{4}{3} \frac{\nu-1}{\nu^{2} z^{2}}-\frac{\left(\phi^{\prime}\right)^{2}}{3} & =0 \\
\left(1-\frac{1}{\nu}\right)\left(3 c z-\frac{4(\nu+1)}{\nu}\right)+z^{-1+\frac{4}{\nu}} e^{-\frac{c z^{2}}{2}} f_{2} q^{2} & =0 \\
\frac{3 c^{2} z^{2}}{2}-c\left(2+\frac{3}{\nu}\right)+\frac{4}{3 z^{2}}\left(2+\frac{3}{\nu}+\frac{1}{\nu^{2}}\right)+\frac{2 e^{\frac{c z^{2}}{2}}}{3 z^{2}} V & =0 \\
\phi^{\prime \prime}+\phi^{\prime}\left(\frac{3 c z}{2}-\frac{\nu+2}{\nu z}\right)+\frac{z^{2} e^{-\frac{c z^{2}}{2}}}{2} \frac{\partial f_{1}}{\partial \phi} A_{t}^{\prime 2}-\frac{z^{-2+\frac{4}{\nu}} e^{-\frac{c z^{2}}{2}}}{2} \frac{\partial f_{2}}{\partial \phi} q^{2}-\frac{e^{\frac{c z^{2}}{2}}}{z^{2}} \frac{\partial V}{\partial \phi} & =0 \\
A_{t}^{\prime \prime}+A_{t}^{\prime}\left(\frac{c z}{2}-\frac{2-\nu}{\nu z}+\frac{f_{1}^{\prime}}{f_{1}}\right) & =0
\end{aligned}
$$

The system (B.29)-(B.34) has the solution:

$$
\begin{aligned}
A_{t}= & \mu, \\
f_{2}= & z^{-\frac{4}{\nu}} \frac{1-\nu}{\nu^{2} q^{2}}\left(3 c z^{2}-4(\nu+1)\right) e^{\frac{c z^{2}}{2}}, \\
\phi= & \frac{1}{2 \sqrt{2} \nu}\left\{\sqrt{3 c^{2} \nu^{2} z^{4}-18 c \nu^{2} z^{2}+8(\nu-1)}-\sqrt{3 c^{2} \nu^{2} z_{*}^{4}-18 c \nu^{2} z_{*}^{2}+8(\nu-1)}+\right. \\
& +2 \sqrt{2(\nu-1)} \ln \left(\frac{z^{2}}{z_{*}^{2}}\right)-3 \sqrt{3} \nu \ln \left(\frac{\sqrt{3 c^{2} \nu^{2} z^{4}-18 c \nu^{2} z^{2}+8(\nu-1)}-\sqrt{3} \nu\left(3-c z^{2}\right)}{\sqrt{3 c^{2} \nu^{2} z_{*}^{4}-18 c \nu^{2} z_{*}^{2}+8(\nu-1)}-\sqrt{3} \nu\left(3-c z_{*}^{2}\right)}\right)- \\
& \left.-2 \sqrt{2(\nu-1)} \ln \left(\frac{9 c \nu^{2} z^{2}-8(\nu-1)-\sqrt{2(\nu-1)} \sqrt{3 c^{2} \nu^{2} z^{4}-18 c \nu^{2} z^{2}+8(\nu-1)}}{9 c \nu^{2} z_{*}^{2}-8(\nu-1)-\sqrt{2(\nu-1)} \sqrt{3 c^{2} \nu^{2} z_{*}^{4}-18 c \nu^{2} z_{*}^{2}+8(\nu-1)}}\right)\right\}, \\
V= & -\frac{1}{4}\left[9 c^{2} z^{4}-6 c z^{2}\left(2+\frac{3}{\nu}\right)+8\left(2+\frac{3}{\nu}+\frac{1}{\nu^{2}}\right)\right] e^{-\frac{c z^{2}}{2}} .
\end{aligned}
$$



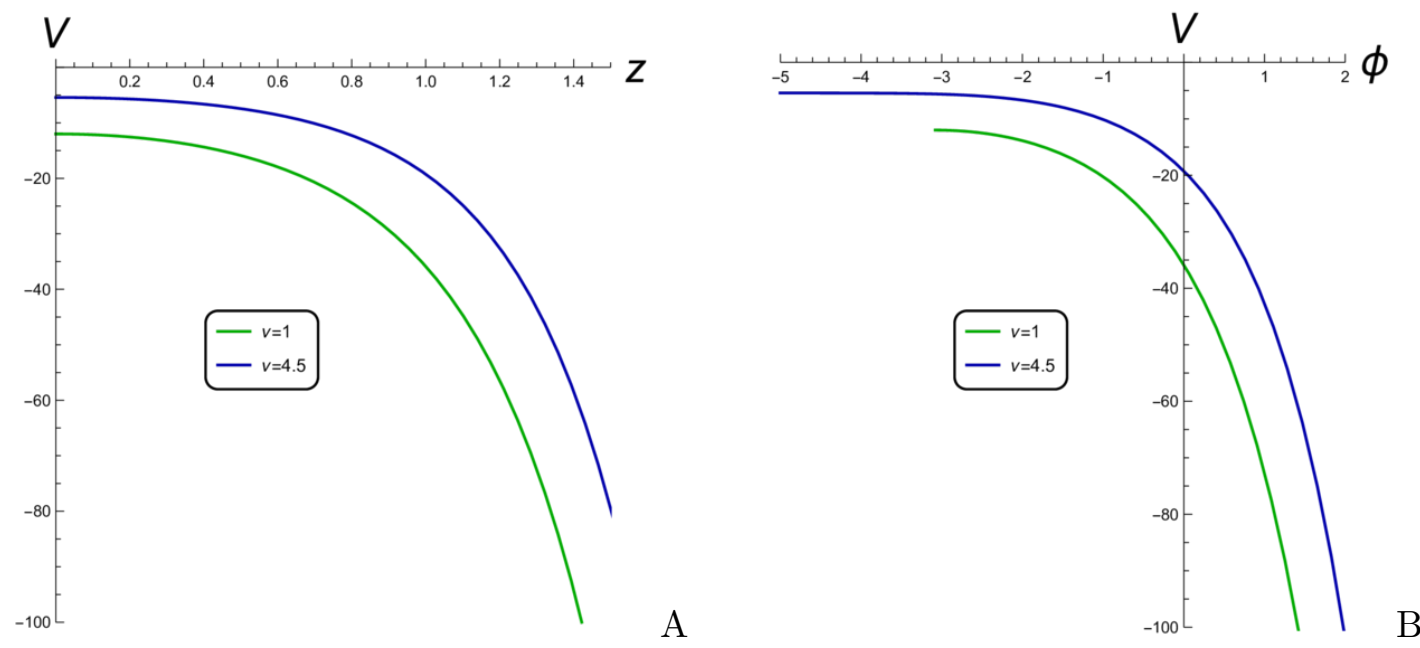

Figure 45. Dilaton potentials that support vacuum solutions corresponding to isotropic and anisotropic cases $\nu=4.5$ with $c=-1$ and initial conditions $\phi(1)=0$ as function of $z \mathrm{~A}$ ) and $\phi \mathrm{B})$.

The particular form of factor $f_{1}$ doesn't matter as it is coupled with the constant function $A_{t}$, so all the terms containing $f_{1}$ also include $A_{t}^{\prime} \equiv 0$. The scalar field $\phi$ isn't influenced by the assumption $g=1$ and coincides with (2.57). The only difference is that $z_{h}$ loses its sense as without the black brane there is no horizon any more. Therefore we should replace $z_{h}$ by some $z_{*}$, whose main property is $\phi\left(z_{*}\right)=0$. The behavior of the scalar potential $V(z)$ and $V(\phi)$ in isotropic and anisotropic cases are compared on figure 45.

If we substitute (B.36)-(B.38) into the equation (B.33) its left-hand side disappears proving that the system (B.29)-(B.34) still remains a self-consistent one.

Open Access. This article is distributed under the terms of the Creative Commons Attribution License (CC-BY 4.0), which permits any use, distribution and reproduction in any medium, provided the original author(s) and source are credited.

\section{References}

[1] H.-T. Ding, F. Karsch and S. Mukherjee, Thermodynamics of strong-interaction matter from Lattice QCD, Int. J. Mod. Phys. E 24 (2015) 1530007 [arXiv:1504.05274] [InSPIRE].

[2] M.A. Stephanov, QCD phase diagram and the critical point, Prog. Theor. Phys. Suppl. 153 (2004) 139 [hep-ph/0402115] [INSPIRE].

[3] J. Casalderrey-Solana, H. Liu, D. Mateos, K. Rajagopal and U.A. Wiedemann, Gauge/String Duality, Hot QCD and Heavy Ion Collisions, arXiv:1101.0618 [INSPIRE].

[4] I.Ya. Aref'eva, Holographic approach to quark-gluon plasma in heavy ion collisions, Phys. Usp. 57 (2014) 527 [INSPIRE].

[5] O. DeWolfe, S.S. Gubser, C. Rosen and D. Teaney, Heavy ions and string theory, Prog. Part. Nucl. Phys. 75 (2014) 86 [arXiv:1304.7794] [INSPIRE].

[6] M. Strickland, Thermalization and isotropization in heavy-ion collisions, Pramana 84 (2015) 671 [arXiv:1312.2285] [INSPIRE]. 
[7] I.Ya. Aref'eva and A.A. Golubtsova, Shock waves in Lifshitz-like spacetimes, JHEP 04 (2015) 011 [arXiv: 1410.4595] [INSPIRE].

[8] ALICE collaboration, Centrality dependence of the charged-particle multiplicity density at midrapidity in $\mathrm{Pb}-\mathrm{Pb}$ collisions at $\sqrt{s_{\mathrm{NN}}}=5.02 \mathrm{TeV}$, Phys. Rev. Lett. 116 (2016) 222302 [arXiv: 1512.06104] [INSPIRE].

[9] S.S. Gubser, S.S. Pufu and A. Yarom, Entropy production in collisions of gravitational shock waves and of heavy ions, Phys. Rev. D 78 (2008) 066014 [arXiv:0805.1551] [InSPIRE].

[10] S.S. Gubser, S.S. Pufu and A. Yarom, Off-center collisions in AdS $S_{5}$ with applications to multiplicity estimates in heavy-ion collisions, JHEP 11 (2009) 050 [arXiv:0902.4062] [INSPIRE].

[11] L. Álvarez-Gaumé, C. Gomez, A. Sabio Vera, A. Tavanfar and M.A. Vazquez-Mozo, Critical formation of trapped surfaces in the collision of gravitational shock waves, JHEP 02 (2009) 009 [arXiv: 0811.3969] [INSPIRE].

[12] S. Lin and E. Shuryak, Grazing Collisions of Gravitational Shock Waves and Entropy Production in Heavy Ion Collision, Phys. Rev. D 79 (2009) 124015 [arXiv:0902.1508] [INSPIRE].

[13] J.L. Albacete, Y.V. Kovchegov and A. Taliotis, Asymmetric Collision of Two Shock Waves in $A d S_{5}$, JHEP 05 (2009) 060 [arXiv:0902.3046] [InSPIRE].

[14] I.Ya. Aref'eva, A.A. Bagrov and E.A. Guseva, Critical Formation of Trapped Surfaces in the Collision of Non-expanding Gravitational Shock Waves in de Sitter Space-Time, JHEP 12 (2009) 009 [arXiv:0905.1087] [InSPIRE].

[15] I.Ya. Aref'eva, A.A. Bagrov and L.V. Joukovskaya, Critical Trapped Surfaces Formation in the Collision of Ultrarelativistic Charges in (A)dS, JHEP 03 (2010) 002 [arXiv:0909.1294] [INSPIRE].

[16] Y.V. Kovchegov and S. Lin, Toward Thermalization in Heavy Ion Collisions at Strong Coupling, JHEP 03 (2010) 057 [arXiv:0911.4707] [INSPIRE].

[17] Y.V. Kovchegov, Shock Wave Collisions and Thermalization in AdS $S_{5}$, Prog. Theor. Phys. Suppl. 187 (2011) 96 [arXiv:1011.0711] [INSPIRE].

[18] E. Kiritsis and A. Taliotis, Multiplicities from black-hole formation in heavy-ion collisions, JHEP 04 (2012) 065 [arXiv:1111.1931] [INSPIRE].

[19] I.Ya. Aref'eva, E.O. Pozdeeva and T.O. Pozdeeva, Holographic estimation of multiplicity and

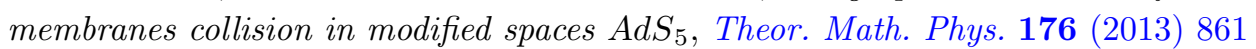
[arXiv: 1401.1180] [INSPIRE].

[20] I.Ya. Aref'eva, E.O. Pozdeeva and T.O. Pozdeeva, Potentials in modified AdS $S_{5}$ spaces with a moderate increase in entropy, Theor. Math. Phys. 180 (2014) 781 [InSPIRE].

[21] D.S. Ageev and I.Ya. Aref'eva, Holographic thermalization in a quark confining background, J. Exp. Theor. Phys. 120 (2015) 436 [arXiv:1409.7558] [INSPIRE].

[22] U. Gürsoy, E. Kiritsis, L. Mazzanti and F. Nitti, Holography and Thermodynamics of 5D Dilaton-gravity, JHEP 05 (2009) 033 [arXiv:0812.0792] [INSPIRE].

[23] D. Mateos and D. Trancanelli, Thermodynamics and Instabilities of a Strongly Coupled Anisotropic Plasma, JHEP 07 (2011) 054 [arXiv:1106.1637] [INSPIRE]. 
[24] D. Mateos and D. Trancanelli, The anisotropic $N=4$ super Yang-Mills plasma and its instabilities, Phys. Rev. Lett. 107 (2011) 101601 [arXiv:1105.3472] [INSPIRE].

[25] M. Chernicoff, D. Fernandez, D. Mateos and D. Trancanelli, Drag force in a strongly coupled anisotropic plasma, JHEP 08 (2012) 100 [arXiv: 1202.3696] [INSPIRE].

[26] M. Chernicoff, D. Fernandez, D. Mateos and D. Trancanelli, Jet quenching in a strongly coupled anisotropic plasma, JHEP 08 (2012) 041 [arXiv:1203.0561] [INSPIRE].

[27] M. Chernicoff, D. Fernandez, D. Mateos and D. Trancanelli, Quarkonium dissociation by anisotropy, JHEP 01 (2013) 170 [arXiv:1208.2672] [INSPIRE].

[28] L. Patino and D. Trancanelli, Thermal photon production in a strongly coupled anisotropic plasma, JHEP 02 (2013) 154 [arXiv:1211.2199] [INSPIRE].

[29] D. Giataganas, Observables in Strongly Coupled Anisotropic Theories, PoS (Corfu2012) 122 [arXiv: 1306.1404] [INSPIRE].

[30] V. Jahnke, A. Luna, L. Patiño and D. Trancanelli, More on thermal probes of a strongly coupled anisotropic plasma, JHEP 01 (2014) 149 [arXiv:1311.5513] [INSPIRE].

[31] I.Ya. Aref'eva, Formation time of quark-gluon plasma in heavy-ion collisions in the holographic shock wave model, Teor. Mat. Fiz. 184 (2015) 398 [arXiv:1503.02185] [INSPIRE].

[32] D. Ávila, D. Fernández, L. Patiño and D. Trancanelli, Thermodynamics of anisotropic branes, JHEP 11 (2016) 132 [arXiv:1609.02167] [INSPIRE].

[33] I.Ya. Aref'eva, A.A. Golubtsova and E. Gourgoulhon, Analytic black branes in Lifshitz-like backgrounds and thermalization, JHEP 09 (2016) 142 [arXiv:1601.06046] [INSPIRE].

[34] I. Aref'eva, Multiplicity and theremalization time in heavy-ions collisions, EPJ Web Conf. 125 (2016) 01007 [INSPIRE].

[35] I. Aref'eva, Holography for Heavy Ions Collisions at LHC and NICA, EPJ Web Conf. 164 (2017) 01014 [arXiv: 1612.08928] [INSPIRE].

[36] D. Giataganas, U. Gürsoy and J.F. Pedraza, Strongly-coupled anisotropic gauge theories and holography, arXiv:1708.05691 [INSPIRE].

[37] O. Andreev and V.I. Zakharov, Heavy-quark potentials and AdS/QCD, Phys. Rev. D 74 (2006) 025023 [hep-ph/0604204] [INSPIRE].

[38] A. Karch, E. Katz, D.T. Son and M.A. Stephanov, Linear confinement and AdS/QCD, Phys. Rev. D 74 (2006) 015005 [hep-ph/0602229] [INSPIRE].

[39] C.D. White, The Cornell potential from general geometries in AdS / QCD, Phys. Lett. B 652 (2007) 79 [hep-ph/0701157] [INSPIRE].

[40] H.J. Pirner and B. Galow, Strong Equivalence of the AdS-Metric and the QCD Running Coupling, Phys. Lett. B 679 (2009) 51 [arXiv:0903.2701] [InSPIRE].

[41] A. Karch, E. Katz, D.T. Son and M.A. Stephanov, On the sign of the dilaton in the soft wall models, JHEP 04 (2011) 066 [arXiv: 1012.4813] [INSPIRE].

[42] O. Andreev, Cold Quark Matter, Quadratic Corrections and Gauge/String Duality, Phys. Rev. D 81 (2010) 087901 [arXiv: 1001.4414] [INSPIRE].

[43] P. Colangelo, F. Giannuzzi and S. Nicotri, Holography, Heavy-Quark Free Energy and the QCD Phase Diagram, Phys. Rev. D 83 (2011) 035015 [arXiv: 1008.3116] [INSPIRE]. 
[44] D. Li, S. He, M. Huang and Q.-S. Yan, Thermodynamics of deformed AdS $S_{5}$ model with a positive/negative quadratic correction in graviton-dilaton system, JHEP 09 (2011) 041 [arXiv: 1103.5389] [INSPIRE].

[45] S. He, S.-Y. Wu, Y. Yang and P.-H. Yuan, Phase Structure in a Dynamical Soft-Wall Holographic QCD Model, JHEP 04 (2013) 093 [arXiv: 1301.0385] [INSPIRE].

[46] Y. Yang and P.-H. Yuan, Confinement-deconfinement phase transition for heavy quarks in a soft wall holographic QCD model, JHEP 12 (2015) 161 [arXiv:1506.05930] [INSPIRE].

[47] R.-G. Cai, S. He and D. Li, A hQCD model and its phase diagram in Einstein-Maxwell-Dilaton system, JHEP 03 (2012) 033 [arXiv:1201.0820] [INSPIRE].

[48] R.-G. Cai, S. Chakrabortty, S. He and L. Li, Some aspects of QGP phase in a hQCD model, JHEP 02 (2013) 068 [arXiv: 1209.4512] [INSPIRE].

[49] M.-W. Li, Y. Yang and P.-H. Yuan, Approaching Confinement Structure for Light Quarks in a Holographic Soft Wall QCD Model, Phys. Rev. D 96 (2017) 066013 [arXiv:1703.09184] [INSPIRE].

[50] A. Chamblin, R. Emparan, C.V. Johnson and R.C. Myers, Charged AdS black holes and catastrophic holography, Phys. Rev. D 60 (1999) 064018 [hep-th/9902170] [InSPIRE].

[51] O. DeWolfe, S.S. Gubser and C. Rosen, A holographic critical point, Phys. Rev. D 83 (2011) 086005 [arXiv: 1012.1864] [INSPIRE].

[52] O. DeWolfe, S.S. Gubser and C. Rosen, Dynamic critical phenomena at a holographic critical point, Phys. Rev. D 84 (2011) 126014 [arXiv:1108. 2029] [INSPIRE].

[53] J. Knaute, R. Yaresko and B. Kämpfer, Holographic QCD phase diagram with critical point from Einstein-Maxwell-dilaton dynamics, Phys. Lett. B 778 (2018) 419 [arXiv:1702.06731] [INSPIRE].

[54] E. Brehm, Heavy Quarks in Strongly Coupled Non-Conformal Plasmas with Anisotropy, arXiv: 1711.08943 [INSPIRE].

[55] D. Dudal and S. Mahapatra, Thermal entropy of a quark-antiquark pair above and below deconfinement from a dynamical holographic QCD model, Phys. Rev. D 96 (2017) 126010 [arXiv: 1708.06995] [INSPIRE].

[56] I.S. Gradshteyn and I.M. Ryzhik, The incomplete gamma functions, in Table of integrals, Series, an Products, 5-th edition, Academic Press (1994).

[57] I.Y. Aref 'eva, A.A. Golubtsova and G. Policastro, Exact holographic RG flows and the $A_{1} \times A_{1}$ Toda chain, arXiv:1803.06764 [INSPIRE].

[58] E. Kiritsis, W. Li and F. Nitti, Holographic RG flow and the Quantum Effective Action, Fortsch. Phys. 62 (2014) 389 [arXiv:1401.0888] [INSPIRE]. 